\title{
The Chemical Components Identified in Tobacco and Tobacco Smoke Prior to 1954: A Chronology of Classical Chemistry*
}

\author{
by \\ Alan Rodgman ${ }^{1}$ and Thomas A. Perfetti ${ }^{2}$ \\ ${ }^{\prime} 2828$ Birchwood Drive, Winston-Salem, North Carolina, 27103-3410, USA \\ ${ }^{2}$ Perfetti and Perfetti, LLC, 2116 New Castle Drive, Winston-Salem, North Carolina, 27103-5750, USA
}

\section{SUMMARY}

Because of the excellent fractionation and identification technologies developed during the early-1950s, the compositions of tobacco and tobacco smoke, both classified as highly complex mixtures, have been defined more completely than the composition of any other highly complex commercial product such as coffee. By year-end 1953, the many years of research by scientists using classical chemical techniques to define the composition of tobacco and its smoke provided meaningful information on the nature of over 300 tobacco components and fewer than 100 tobacco smoke components. Those involved in the pre-1954 research not only provided the cornerstone of our knowledge of the two compositions but also deserve the gratitude of their successors for the early information generated on tobacco and its smoke. This article is our tribute to those researchers who generated much meaningful knowledge on the composition of tobacco and tobacco smoke prior to 1954 despite the now known fractionation and analytical limitations of the so-called classical chemical techniques. It also notes the similarity of some of the early and more recent research results obtained on the chemical and biological properties of smoke condensate and several of its components from tobacco with those obtained by RoFFO in the 1930s on a destructive distillate of tobacco. [Beitr. Tabakforsch. Int. 23 (2009) 277-333]

\section{ZUSAMMENFASSUNG}

Wegen der hervorragenden Technologien zur Fraktionierung und Identifizierung, die in den frühen 1950iger Jahren entwickelt wurden, wurden die Zusammensetzung von Tabak- und Tabakrauch, die beide als hochkomplexe Mischungen zu klassifizieren sind, umfassender beschrie- ben als jedes andere komplexe, handelsübliche Gemisch, wie zum Beispiel Kaffee. Am Ende des Jahres 1953 lieferten die jahrelangen Forschungsarbeiten von Wissenschaftlern, die die Zusammensetzung des Tabaks und des Tabakrauchs mit klassischen chemischen Analysemethoden untersucht hatten, wichtige Informationen über die Eigenschaften von über 300 Tabakinhaltsstoffen und nahezu 100 Tabakrauchinhaltsstoffe. Die Wissenschaftler, die an der Forschung vor 1954 beteiligt waren, lieferten nicht nur den Grundstein unseres Wissens über beide Gemische, sondern sie verdienen auch den Dank ihrer Nachfolger für den frühen Kenntnisstand zur Zusammensetzung des Tabaks und des Tabakrauchs. Mit diesem Beitrag zollen wir jenen Wissenschaftlern Tribut, die bedeutende Kenntnisse über die chemische Zusammensetzung vor 1954 lieferten, trotz der heute bekannten damals existierenden Grenzen der sogenannten klassischen chemischen Methoden bei der Fraktionierung und Analyse. Diese Arbeit zeigt auch die Ähnlichkeit mancher früherer Ergebnisse zu den chemischen und biologischen Eigenschaften des Rauchkondensats und mehrerer Tabakinhaltsstoffe mit denen von ROFFO aus den 1930iger Jahren über ein gefährliches Destillat des Tabaks. [Beitr. Tabakforsch. Int. 23 (2009) 277-333]

\section{RESUME}

La composition chimique du tabac et de la fumée de tabac, tous les deux classifiés comme des mélanges très complexes, a été étudiée de façon plus complète que la composition de chaque autre mélange complexe commerciale comme le café, à cause des technologies excellentes de fractionnement et de l'identification développées au début des années 1950. A la fin de l'année 1953, les nombreuses années de recherches effectuées par des

*Received: $13^{\text {th }}$ June 2008 - accepted: $4^{\text {th }}$ December 2008 
chercheurs avec des techniques chimiques classiques pour analyser la composition chimique du tabac et de la fumée de tabac, ont fournit des informations compréhensibles sur plus de 300 composants de tabac et près de 100 composants de la fumée de tabac. Les chercheurs des années avant 1954 n'ont pas seulement fournit la base de nos connaissances sur la composition du tabac et de la fumée mais méritent également la reconnaissance de leurs successeurs pour leurs travaux précoce. Avec cet article nous exprimons notre estime aux chercheurs des années avant 1954, qui ont fournit beaucoup d'informations compréhensibles sur la composition du tabac et de la fumée de tabac, malgré les limitations analytiques des techniques chimiques dites classiques. La similarité de quelques résultats, sur les propriétés biologiques et chimiques du condensat de la fumée et plusieurs composants du tabac, obtenues par ROFFO dans les années 1930 sur un distillat nuisible du tabac, est également présentée. [Beitr. Tabakforsch. Int. 23 (2009) 277-333]

\section{INTRODUCTION}

The compilation of the catalogue on the more than 8400 chemical components identified in tobacco and tobacco smoke provided an excellent assessment of the caliber of the research conducted since its escalation in the mid-1950s. The classical chemical analysis used before that date to isolate and characterize a component of a complex mixture was gradually augmented post-1953 by the inclusion of many different, new, and highly efficient separation and analytical technologies. Various types of chromatography coupled with various spectral technologies such as ultraviolet, infrared, nuclear magnetic resonance, and mass provided the means to identify more complex structures of components isolated in much lesser amounts than could be done by using the classical chemical approach. For example, inclusion of such technologies resulted in the increase of the number of identified chemical components in tobacco smoke from the fewer than 100 reported by KOSAK in 1954 (1) to the more than 5200 catalogued recently by RODGMAN and PERFETTI (2). The manifold scientific skills generated and employed during those more than 50 years of research obviously deserve great commendation.

In the early part of the twentieth century until the mid 1950 s there was limited interest in the relationship between the majority of identified chemicals in tobacco and tobacco smoke and the varied asserted health issues associated with smoking in general, compared to the time after the mid1950s. This limited interest may have been due to a lack of understanding of how to interpret the chemical data collected on the complex mixtures of tobacco and tobacco smoke in relation to biological end-points data. On the other hand, early tobacco research concentrated on a greater understanding of the alkaloids in tobacco.

In early tobacco research, nicotine and other related tobacco alkaloids were regarded as toxic constituents of tobacco and their presence in smoke was considered a health concern. Tobacco alkaloids were also important as raw materials for pesticides and as sources of raw material for the chemical industry. As a result, considerable effort was focused in the early years of tobacco research on nicotine and related tobacco alkaloids. This work included the development of new analytical determinations for tobacco alkaloids and tests to measure its toxicity and dependence of nicotine for smokers. Work in the area of breeding new tobacco lines was focused on two directions. Efforts were directed at developing low nicotine or nicotine-free tobaccos for health reasons, and secondly in the direction of tobaccos extremely high in nicotine, as a raw material for pesticides, chemicals, and pharmaceuticals. During our cataloguing of the chemical components of tobacco and tobacco smoke, it became apparent that the chemical research conducted on them from early in the 1800s to September 1953, the date of submission of the KOSAK manuscript for publication, also deserved considerable commendation. Considerable skill in classical chemical methods was used to isolate and characterize the tobacco and smoke components known by late 1953 . In this article, the results of that research are summarized in an attempt to illustrate that not only should they not be minimized or disregarded but also the investigators who generated such meaningful results deserve considerable credit.

One of the stimuli cited by KOSAK (1) for the generation of his 1954 catalogue of tobacco smoke components was the brief 1952 published report by GRAHAM et al. (3) on the induction of tumors in laboratory animals repeatedly painted with large doses of cigarette smoke tar, a report that was followed in early 1953 by a presentation at the American Association for Cancer Research (4) and then by a detailed publication in a peer-reviewed scientific journal in late 1953 (5). WYNDER et al. (5) described many of the early studies on the effects of exposure of laboratory animals to various tars, including those derived from tobacco by smoking or extraction [E. HoFFMANN et al. (6), HELWIG (7, 8), BOGEN and LOOMIS (9), COOPER et al. (10), CAMPBELL $(11,12)$, SCHÜRCH and WinTERSTEIN (13), TAKI (14), Sugiura (15), Flory $(16,17)$, and Shubik (18)]. An early study reported in 1911 by WACKER and SCHMINCKE (19) preceded the development and description of the procedure to induce cancer in laboratory animals by skin painting with coal tar in 1915-1918 by YAMAGIWA and ICHIKAWA (20, 21). As noted by WYNDER et al. (5), each of the tobacco tar studies conducted after The YAMAGIWA-ICHIKAWA reports suffered from one or more deficiencies such as a low number of daily or weekly paintings, low tar-painting dosage, inadequate smoking procedure for tar collection, and the like. However, seldom discussed is the fact that the WYNDER et al. study involved a cigarette smoking procedure (35-mL puff, 2-sec puff, 3 puff/min) different from the one in vogue since its elucidation $(35-\mathrm{mL}$ puff, $2-\mathrm{sec}$ puff, 1 puff/min) by BRADFORD et al. in 1936 (22). Tripling the puff/min not only substantially increases the per cigarette tar yield but also drastically alters its composition, with substantial increases in several smoke components with known tumorigenicity to mouse skin, e.g., the polycyclic aromatic hydrocarbons (PAHs) benzo $[a]$ pyrene $(\mathrm{B}[a] \mathrm{P})$ and dibenz $[a, h]$ anthracene $(\mathrm{DB}[a, h] \mathrm{A})$.

Other reports that triggered interest in the biological properties and chemical composition of cigarette smoke condensate (CSC) were the numerous publications between 1950 and 1953 on the epidemiology of lung cancer and cigarette smoking. They were presented in 1950 by WYNDER and GRAHAM (23), DOLL and Hill (24), LeVIN et al. (25), 
MiLlS and PORTER (26), and SCHREK et al. (27). These were followed in 1952 and 1953 by similar extensive studies conducted by DOLL and HILL (28), MCCONNELL et al. (29), KOULUMIES (30), LiCKINT (31), and SADOWSKY et al. (32). It should be noted that the prospective method did not replace retrospective studies. Both approaches have their advantages and disadvantages. In the retrospective study, interviewers record the habits of smokers and controls; the prospective studies are better suited to survey larger groups of diseased and controls in terms of their environment, their behaviors, and specific diseases they contract.

Preceding these 1950-1953 epidemiological studies were several reported from 1912 to 1950 but, in their 1950 report, GRAHAM and WYNDER (23) described in general several of the deficiencies of the earlier epidemiological studies, e.g., small sample size, lack of a control population, statistical procedure, tumor definition. Among the earlier studies were reports and/or comments on respiratory tract cancer by ADLER (33) in 1912, by TYLECOTE (34) in 1927, by LICKINT (35) in 1935, by ARKIN and WAGNER (36) in 1936, by KENNAWAY and KENNAWAY $(37,38)$ in 1936 and 1947, by ROFFO (39) in 1937, by MÜLLER (40) in 1939 , by OCHSNER and DEBAKEY $(41,42)$ in 1940 and 1941, and by SCHAIRER and SCHÖNINGER (43) in 1943.

The reports by OCHSNER and DEBAKEY subsequently led to an interesting situation in the content of several U.S. Surgeon Generals' reports on smoking and health. From the late 1930s through the late 1960s, OCHSNER, at that time one of the few eminent lung cancer surgeons in the U.S., authored or co-authored over 40 articles and three books in which it was repeatedly asserted that the major cause of lung cancer was cigarette smoking. Despite his number of publications between the late 1930s and late 1963, the 1964 Advisory Committee to the U.S. Surgeon General (44) and the 1979 (45) and 1982 (46) U.S. Surgeon Generals cited only one 1939 OCHSNER-authored publication (47) and one of his authored books, the 1954 edition (48) on the relationship between lung cancer and cigarette smoking. Was the limitation of the citations of OCHSNER's publications on cigarette smoking and lung cancer possibly due to either or both of the following situations?

- Despite the repeated assertion that the major cause of lung cancer was cigarette smoking, OCHSNER and his colleagues frequently included other comments in the same articles, e.g., a) The cause of the increasing incidence of cancer of the lung is not definite [see p. 212 in (42)], b) The etiology of bronchogenic carcinoma is unknown. (49), c) The etiological picture [for bronchogenic carcinoma] is obscure. It is probable that there are a number of etiological factors in the production of this disease (50).

- Despite the repeated assertion that the major cause of lung cancer was cigarette smoking, the data on patients undergoing lung resection in the Ochsner Clinic because of lung cancer were summarized in several OCHSNER et al. reports as follows: a) In 129 resected cases, no factor was found which might bear a significant relationship to the occurrence of the disease. Neither occupation nor smoking habits, which some reports, including our own, have stressed as of possible etiological significance seems of any special significance in this particular series (51). b) In the analysis of 147 resected cases, no etiological factor was found to bear a significant relationship to the occurrence of the disease. Both occupation and smoking, which had been particularly emphasized by some observers as possible etiological factors and which we were inclined previously to consider more seriously, were found to have no special significance in this analysis (52).

\section{CHEMICAL COMPONENTS OF TOBACCO AND TOBACCO SMOKE IDENTIFIED PRIOR TO 1954}

Many components were identified in tobacco and/or tobacco smoke prior to the issuance of the 1954 KOSAK report (1), the numerous epidemiological studies on tobacco smoking and respiratory cancer, and the 1953 report of tumor induction in laboratory animals by cigarette-tar painting (5). Most of the pre-1954 characterizations of such components were accomplished by the so-called "classical chemical procedures." The components are listed in Table 1 with the identification and confirmation listed chronologically. If more than one study on a specific component was reported in a given year, the investigators in that year are listed alphabetically.

Examination of the data in Table 1 reveals the many investigators who contributed much meaningful information on tobacco and/or smoke composition prior to yearend 1953. They include the following: BRÜCKNER, BURKHARD, EULENBERG, FrANKENBURG, GABELYA, GARNER, GotTscho, Kipriyanov, KisSling, KoBel, LeHMANN, Molinari, Neuberg, Pfyl, Pontag, Preiss, Pyriki, ROFFO, SchÖller, SHMUK, SpÄTH, ThOMS, VoHL, and WENUSCH. Each of them deserves much tribute for their contributions. Several of them continued to contribute to our knowledge of the composition of tobacco and/or tobacco smoke after 1953, e.g., FRANKENBURG, GARNER, GOTTSCHO, and PYRIKI.

Compared to the fewer than 100 tobacco smoke components listed by KOSAK (1), Table 1 contains 325 chemical components identified and studied in tobacco prior to the publication of the KOSAK article. Included in 383 entries in Table 1 (as they were in the KoSAK tabulation) are several components originally assumed to be individual alkaloidrelated components but subsequently were found to be known compounds or mixtures of known compounds, e.g., anodmine, gudham, lathrein, lohitam, obelin, poikiline, $\alpha$ socratine, $\beta$-socratine, and $\gamma$-socratine $(53,54,55,56,57)$. They were not included in the count of the 325 identified tobacco components. The eventual characterization of anodmine, gudham, lathrein, lohitam, obelin, and the three socratines was described in 1955 by KUFFNER et al. (58). Their characterization of these supposedly alkaloid-related components was summarized by JOHNSTONE and PLIMMER (59) in their 1959 review of tobacco and tobacco smoke composition. They wrote in 1959:

The constitution of these bases remained unknown until recently when investigators having access to original specimens were able to elucidate the identities of some of them by application of modern analytical techniques. $\gamma$ socratine was found to be identical with $l$-nornicotine, and a crude mixture of $\alpha$ - and $\beta$-socratine (the only sample available), was shown to consist mainly of nicotyrine and 2,3'-dipyridyl with small quantities of nicotinic acid, nornicotine, and possibly anatabine (58). 
Table 1. Chronology from 1800 to late 1953 of identified items in tobacco, tobacco smoke, and tobacco distillate

\begin{tabular}{|c|c|c|c|}
\hline CAS No. & Name (per CA Collective Index) & $\begin{array}{l}\text { Tobacco smoke and/or tobacco } \\
\text { distillate [dry (Dr), destructive (D)] }\end{array}$ & Tobacco \\
\hline \multirow[t]{2}{*}{$75-07-0$} & Acetaldehyde & $\begin{array}{l}1908 \text { Brasch and Neuberg (64) } \\
1909 \text { Brasch }(65) \\
1931 \text { Neuberg and Burkard (66) } \\
1933 \text { Pfyl }(67) \\
1954 \text { Kosak (1) }\end{array}$ & $\begin{array}{l}1926 \text { Neuberg and Kobel (68) } \\
1931 \text { Neuberg and Burkard (66) } \\
1936 \text { Dixon et al. (69) }\end{array}$ \\
\hline & Acetate & $\begin{array}{l}1939 \text { Roffo }(70,71,72,73)(D) \\
1954 \text { Kosak (1) }\end{array}$ & \\
\hline \multirow[t]{5}{*}{$64-19-7$} & Acetic acid & $\begin{array}{l}1843 \text { Zeise (74) (Dr) } \\
1871 \text { Vohl and Eulenberg }(75,76) \\
1892 \text { Abeles and Paschkis }(77) \\
1929 \text { Gabelya and Kipriyanov (78) (Dr) } \\
1931 \text { Neuberg and Burkard (66) } \\
1937 \text { Bradford et al. (79) } \\
1939 \text { Roffo }(70,71,72,73)(D) \\
1950 \text { Peterson (80) } \\
1951 \text { Garner (81) } \\
1952 \text { James and Martin (82) } \\
1954 \text { Kosak (1) }\end{array}$ & $\begin{array}{l}1871 \text { Vohl and Eulenberg (75) } \\
1884 \text { Takayama }(83) \\
1909 \text { Garner }(84) \\
1924 \text { Shmuk (85) } \\
1929 \text { Balabucha-Popzova (86) } \\
1929 \text { Shmuk (87) } \\
1931 \text { Yamafuji (88) } \\
1935 \text { Koenig (89) } \\
1936 \text { Dixon et al. (69) } \\
1941 \text { Sabetay et al. (90) } \\
1951 \text { Garner (81) }\end{array}$ \\
\hline & Acids, aliphatic & $\begin{array}{l}1931 \text { Neuberg and Burkard (66) } \\
1937 \text { Wenusch and Schöller (91) } \\
1940 \text { Haag (92) } \\
1954 \text { Kosak (1) }\end{array}$ & $\begin{array}{l}1930 \text { Shmuk and Piatnicki (93) } \\
1931 \text { Yamafuji (88) } \\
1953 \text { Wada and Kobashi (94) } \\
1943 \text { Venkatarao et al. (95) }\end{array}$ \\
\hline & Acids, amino- & & $\begin{array}{l}1951 \text { Roberts and Wood (96) } \\
1952 \text { Frankenburg and Gottscho (97) } \\
1953 \text { Pearse and Novellie (98) } \\
1953 \text { Zacharius and Frankenburg (99) }\end{array}$ \\
\hline & Acids, non-volatile & & $\begin{array}{l}1914 \text { Garner et al. (100) } \\
1931 \text { Vickery and Pucher (101) } \\
1933 \text { Pucher and Vickery (102) } \\
1951 \text { Garner (81) }\end{array}$ \\
\hline & Acids, phenolic & $\begin{array}{l}1939 \text { Roffo }(8,9,10,11)(D) \\
1954 \text { Kosak (1) }\end{array}$ & \\
\hline $7440-34-8$ & Actinium & & 1937 Drobkov (103) \\
\hline $56-41-7$ & $L-\alpha-$ Alanine & & $\begin{array}{l}1952 \text { Frankenburg and Gottscho (97) } \\
1953 \text { Pearse and Novellie (98) } \\
1953 \text { Zacharius and Frankenburg (99) }\end{array}$ \\
\hline \multirow[t]{3}{*}{$107-95-9$} & $\beta$-Alanine & & $\begin{array}{l}1953 \text { Pearse and Novellie (98) } \\
1953 \text { Zacharius and Frankenburg (99) }\end{array}$ \\
\hline & Aldehydes & & 1953 Ross (104) \\
\hline & $\begin{array}{l}\text { Alkaloids, tobacco or tobacco } \\
\text { smoke }\end{array}$ & $\begin{array}{l}1931 \text { Shmuk and Kolesnik (105) } \\
1939 \text { Wenusch }(106) \\
1953 \text { Latimer (107) }\end{array}$ & $\begin{array}{l}1901 \text { Pictet and Rotschy }(108,109) \\
1908 \text { Pictet and Court (110) } \\
1931 \text { Ehrenstein (111) } \\
1938 \text { Marion (112) } \\
1939 \text { Späth and Kuffner (113) } \\
1941 \text { Jackson (114) } \\
1948 \text { Frankenburg (115) } \\
1952 \text { Badgett et al. (116) } \\
1953 \text { Tso and Jeffrey (117) }\end{array}$ \\
\hline \multirow[t]{2}{*}{$7429-90-5$} & Aluminum & 1952 Bailey (118) & $\begin{array}{l}1932 \text { Eisenmenger (119) } \\
1938 \text { McMurtrey and Robinson (120) } \\
1938 \text { Morgan and Street (121) } \\
1944 \text { LeCompte (122) } \\
1951 \text { Garner (81) }\end{array}$ \\
\hline & Amines, aliphatic & 1929 Gabelya and Kipriyanov (78) (Dr) & \\
\hline $7664-41-7$ & Ammonia & $\begin{array}{l}1857 \text { Vogel }(123,124) \\
1858 \text { Vogel }(125) \\
1871 \text { Vohl and Eulenberg }(75,76) \\
1879 \text { Périgord }(126) \\
1880 \text { LeBon }(127) \\
1899 \text { Thoms }(128) \\
1900 \text { Thoms }(129) \\
1902 \text { Pontag }(130) \\
1903 \text { Pontag }(131) \\
1908 \text { Biederbeck }(132) \\
1908 \text { Lee }(133) \\
1908 \text { Lehmann }(134) \\
1909 \text { Lehmann }(135)\end{array}$ & $\begin{array}{l}1885 \text { Müller-Thurgen (153) } \\
1894 \text { Behrens (154) } \\
1908 \text { Pictet and Court (110) } \\
1914 \text { Garner et al. (100) } \\
1928 \text { Shmuk (140) } \\
1929 \text { Gabelya and Kipriyanov (78) } \\
1930 \text { Smirnov and Izvoshtshikov (55) } \\
1936 \text { Dixon et al. (69) } \\
1936 \text { Preiss (156) } \\
1937 \text { Fromm (157) } \\
1939 \text { Gaertner (158) } \\
1939 \text { Shmuk (165) } \\
1948 \text { Pyriki (166) }\end{array}$ \\
\hline
\end{tabular}


Table 1. (cont.)

\begin{tabular}{|c|c|c|c|}
\hline CAS No. & Name (per CA Collective Index) & $\begin{array}{l}\text { Tobacco smoke and/or tobacco } \\
\text { distillate [dry (Dr), destructive (D)] }\end{array}$ & Tobacco \\
\hline \multirow[t]{27}{*}{$7664-41-7$} & Ammonia (cont.) & 1910 Toth and Krampera (136) & 1950 Molinari (159) \\
\hline & & 1911 Vaubel (137) & 1951 Garner (81) \\
\hline & & 1912 Anonymous $(138,139)$ & 1952 Hough et al. (160) \\
\hline & & 1928 Shmuk (140) & 1952 Jensen $(161)$ \\
\hline & & 1929 Bogen (141) & \\
\hline & & 1929 Gabelya and Kipriyanov (78) (Dr) & \\
\hline & & 1929 Koperina (142) & \\
\hline & & 1931 Gavrilov and Koperina (143) & \\
\hline & & 1931 Haley et al. (144) & \\
\hline & & 1931 Shmuk and Kolesnik (105) & \\
\hline & & 1932 Barta and Toole $(145,146)$ & \\
\hline & & 1932 McNally (147) & \\
\hline & & 1933 Pfyl (148) & \\
\hline & & 1934 Barta (149) & \\
\hline & & 1936 Bogen (150) & \\
\hline & & 1936 Preiss $(151,152,156)$ & \\
\hline & & 1937 Bradford et al. (79) & \\
\hline & & $1939 \operatorname{Dittmar}(162,163,164)$ & \\
\hline & & 1939 Roffo $(70,71,72,73)$ (D) & \\
\hline & & 1939 Shmuk (165) & \\
\hline & & 1948 Pyriki (166) & \\
\hline & & 1950 Peterson (80) & \\
\hline & & 1951 Garner (81) & \\
\hline & & 1952 Larsen (167) & \\
\hline & & 1954 Kosak (1) & \\
\hline & Ammonium salts & 1936 Cuvelier (168) & \\
\hline & Ammoresinol & & 1936 Späth and Zajic (169) \\
\hline \multirow[t]{9}{*}{$9000-92-4$} & Amylase & & 1913 Oosthuizen and Shedd (170) \\
\hline & & & 1937 Matsumina (171) \\
\hline & & & 1942 Ward (172) \\
\hline & & & 1946 Garner (173) \\
\hline & & & 1951 Garner (81) \\
\hline & & & 1951 Nakai and Inaba (174) \\
\hline & & & 1953 Barrett et al. (175) \\
\hline & Anabaseine & & 1939 Henry (176) \\
\hline & $\begin{array}{l}\text { Anodmin }=\text { mixture of basic } \\
\text { components of tobacco and its } \\
\text { smoke }(58)\end{array}$ & $\begin{array}{l}1935 \text { Wenusch and Schöller }(55,56) \\
1936 \text { Wenusch and Schöller }(177) \\
1954 \text { Kosak (1) }\end{array}$ & 1935 Wenusch and Schöller $(55,56)$ \\
\hline $120-12-7$ & Anthracene & $\begin{array}{l}1939 \text { Roffo }(70,71,72,73,178)(D) \\
1953 \text { Cooper and Lindsey }(179) \\
1954 \text { Kosak }(1)\end{array}$ & \\
\hline $7440-36-0$ & Antimony & & 1934 Heffer et al. (180) \\
\hline $147-81-9$ & Arabinose & & 1929 Gabelya and Kipriyanov (181) \\
\hline \multirow[t]{5}{*}{$7004-12-8$} & Arginine & & 1933 Vickery et al. (182) \\
\hline & & & 1935 Vickery et al. (183) \\
\hline & & & 1951 Garner (81) \\
\hline & & & 1953 Pearse and Novellie (98) \\
\hline & & & 1953 Zacharius and Frankenburg (99) \\
\hline \multirow[t]{20}{*}{$7440-38-2$} & Arsenic & 1922 Leitch and Kennaway (184) & 1905 Boening (195) \\
\hline & & 1927 Remington (185) & 1922 Leitch and Kennaway (184) \\
\hline & & 1932 McNally (147) & 1927 Remington (185) \\
\hline & & 1934 Gross and Nelson (186) & 1928 Popp $(196,197)$ \\
\hline & & 1935 Bastedo (187) & 1934 Carey et al. (198) \\
\hline & & 1945 Thomas and Collier (188) & 1935 McMurtrey (199) \\
\hline & & 1947 Griffon and Delga (189) & 1938 McMurtrey (200) \\
\hline & & 1950 Daff and Kennaway $(190,191)$ & 1939 Barksdale (201) \\
\hline & & 1951 Daff et al. (192) & 1940 Barksdale (202) \\
\hline & & 1952 Goulden et al. (193) & 1941 McMurtrey (203) \\
\hline & & 1953 Monnet and Dupont (194) & 1942 Vucetich and Carratala (204) \\
\hline & & 1954 Kosak (1) & 1944 Vincent (205) \\
\hline & & & 1947 Griffon and Delga (189) \\
\hline & & & 1950 Daff and Kennaway $(190,191)$ \\
\hline & & & 1951 Daff et al. (192) \\
\hline & & & 1951 Garner (81) \\
\hline & & & 1951 Oliver (206) \\
\hline & & & 1952 Bunce (207) \\
\hline & & & 1953 Monnet and Dupont (194) \\
\hline & & & 1953 Wolff et al. (208) \\
\hline
\end{tabular}


Table 1. (cont.)

\begin{tabular}{|c|c|c|c|}
\hline CAS No. & Name (per CA Collective Index) & $\begin{array}{l}\text { Tobacco smoke and/or tobacco } \\
\text { distillate [dry (Dr), destructive (D)] }\end{array}$ & Tobacco \\
\hline $1327-53-3$ & Arsenic oxide $\left(\mathrm{As}_{2} \mathrm{O}_{3}\right)$ & $\begin{array}{l}1927 \text { Remington (185) } \\
1932 \text { McNally (147) } \\
1934 \text { Gross and Nelson (186) } \\
1935 \text { Bastedo (187) } \\
1945 \text { Thomas and Collier (188) } \\
1947 \text { Griffon and Delga }(189) \\
1950 \text { Daff and Kennaway }(190,191) \\
1951 \text { Daff et al. (192) } \\
1952 \text { Goulden et al. (193) } \\
1953 \text { Monnet and Dupont (194) } \\
1954 \text { Kosak (1) }\end{array}$ & $\begin{array}{l}1905 \text { Boening (195) } \\
1927 \text { Remington (185) } \\
1928 \text { Popp }(196,197) \\
1934 \text { Carey et al. }(198) \\
1935 \text { McMurtrey (199) } \\
1938 \text { McMurtrey }(200) \\
1940 \text { Barksdale }(202) \\
1941 \text { McMurtrey }(203) \\
1942 \text { Vucetich and Carratala }(204) \\
1947 \text { Griffon and Delga }(189) \\
1950 \text { Daff and Kennaway }(190,191) \\
1951 \text { Daff et al. (192) } \\
1951 \text { Garner }(81) \\
1953 \text { Monnet and Dupont (194) }\end{array}$ \\
\hline & Arsenical insecticides & & $\begin{array}{l}1901 \text { Marlatt }(209) \\
1908 \text { Marlatt }(210)\end{array}$ \\
\hline $50-81-7$ & Ascorbic acid & & $\begin{array}{l}1947 \text { Maton (211) } \\
1951 \text { Lona and Porzio-Giovanola (212) } \\
1951 \text { Tombesi (213) }\end{array}$ \\
\hline $7006-34-0$ & Asparagine & & $\begin{array}{l}1894 \text { Behrens (154) } \\
1937 \text { Vickery et al. (214) } \\
1951 \text { Garner ( } 81) \\
1952 \text { Frankenburg and Gottscho (97) } \\
1953 \text { Pearse and Novellie (98) } \\
1953 \text { Zacharius and Frankenburg (99) }\end{array}$ \\
\hline $56-84-8$ & Aspartic acid & & $\begin{array}{l}1952 \text { Frankenburg and Gottscho (97) } \\
1953 \text { Pearse and Novellie (98) } \\
1953 \text { Zacharius and Frankenburg (99) }\end{array}$ \\
\hline $275-51-4$ & Azulene & $\begin{array}{l}1947 \text { Ikeda }(215) \\
1954 \text { Kosak (1) }\end{array}$ & \\
\hline $7440-39-3$ & Barium & & $\begin{array}{l}1913 \text { McHargue }(216) \\
1913 \text { Traetta-Mosca }(217) \\
1916 \text { Artis and Maxwell }(218) \\
1916 \text { Knight }(219) \\
1921 \text { Headden }(220)\end{array}$ \\
\hline & Bases & 1940 Haag (92) & $\begin{array}{l}1931 \text { Yamafuji (88) } \\
1934 \text { Nito and Kitamura (221) }\end{array}$ \\
\hline \multirow[t]{5}{*}{$100-52-7$} & Benzaldehyde & $\begin{array}{l}1931 \text { Neuberg and Burkard (66) } \\
1954 \text { Kosak (1) }\end{array}$ & \\
\hline & Benzenamine, alkyl- & & 1871 Vohl and Eulenberg (75) \\
\hline & Benzenamine, alkyl- & & 1871 Vohl and Eulenberg (75) \\
\hline & Benzenamine, alkyl- & & 1871 Vohl and Eulenberg (75) \\
\hline & $\begin{array}{l}\text { Benzenamine, 4-(1,1- } \\
\text { dimethylethyl)- }\end{array}$ & & 1871 Vohl and Eulenberg (75) \\
\hline $53-19-0$ & $\begin{array}{l}\text { Benzene, 1-chloro-2-[2,2-dichloro- } \\
\text { 1-(4-chlorophenyl)ethyl]- } \\
\{\text { \{o,p'-DDD; o,p'-TDE }\}\end{array}$ & & 1951 Vinzant (60) \\
\hline $4329-12-8$ & $\begin{array}{l}\text { Benzene, 1-chloro-3-[2,2-dichloro- } \\
\text { 1-(4-chlorophenyl)ethyl]- } \\
\qquad\left\{m, p^{\prime}-\mathrm{DDD}\right\}\end{array}$ & & 1951 Vinzant (60) \\
\hline $789-02-6$ & $\begin{array}{r}\text { Benzene, 1-chloro-2-[2,2,2- } \\
\text { trichloro-1-(4-chlorophenyl)ethyl]- } \\
\left\{0, p^{\prime}-\mathrm{DDT}\right\}\end{array}$ & & 1951 Vinzant (60) \\
\hline $50-29-3$ & $\begin{array}{l}\text { Benzene, } 1,1^{\prime}-(2,2,2- \\
\text { trichloroethylidene)bis[4-chloro- } \\
\left\{p, p^{\prime}-\mathrm{DDT}\right\}\end{array}$ & & 1951 Vinzant (60) \\
\hline $100-21-0$ & $\begin{array}{l}\text { 1,4-Benzenedicarboxylic acid } \\
\quad \text { terephthalic acid\} }\end{array}$ & & 1946 Frankenburg (222) \\
\hline $120-80-9$ & 1,2-Benzenediol & $\begin{array}{l}1893 \text { Kissling (223) } \\
1936 \text { Molinari }(224) \\
1950 \text { Molinari (159) }\end{array}$ & 1935 Koenig (89) \\
\hline $123-31-9$ & 1,4-Benzenediol $\quad\{$ hydroquinone & & 1952 Volgunov (225) \\
\hline $100-51-6$ & Benzenemethanol $\{$ benzyl alcohol\} & 1939 Wenusch (226) & 1939 Wenusch (226) \\
\hline $65-85-0$ & $\begin{array}{l}\text { Benzoic acid } \\
\quad\{\text { benzenecarboxylic acid\} }\end{array}$ & $\begin{array}{l}1931 \text { Neuberg and Burkard (66) } \\
1939 \text { Wenusch }(226) \\
1940 \text { Haag }(92) \\
1954 \text { Kosak (1) }\end{array}$ & \\
\hline
\end{tabular}




\begin{tabular}{|c|c|c|c|}
\hline CAS No. & Name (per CA Collective Index) & $\begin{array}{l}\text { Tobacco smoke and/or tobacco } \\
\text { distillate [dry (Dr), destructive (D)] }\end{array}$ & Tobacco \\
\hline $99-50-3$ & $\begin{array}{l}\text { Benzoic acid, 3,4-dihydroxy- } \\
\text { \{protocatechuic acid\} }\end{array}$ & & 1929 Shmuk (87) \\
\hline $149-91-7$ & $\begin{array}{l}\text { Benzoic acid, 3,4,5-trihydroxy- } \\
\text { \{gallic acid\} }\end{array}$ & & 1929 Shmuk (87) \\
\hline $59-02-9$ & 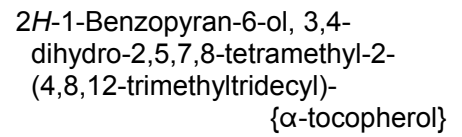 & & 1945 Riemenschneider et al. (227) \\
\hline $92-61-5$ & $\begin{array}{l}\text { 2H-1-Benzopyran-2-one, } 7- \\
\text { hydroxy-6-methoxy- } \\
\quad\{\text { scopoletin\} }\end{array}$ & & $\begin{array}{l}1948 \text { Best (228) } \\
1953 \text { Johanson (229) }\end{array}$ \\
\hline $21637-25-2$ & $\begin{array}{l}\text { 4H-1-Benzopyran-4-one, 2-(3,4- } \\
\text { dihydroxyphenyl)-3-( } \beta-D- \\
\text { glucofuranosyloxy)-5,7-dihydroxy- } \\
\text { \{isoquercitrin\} }\end{array}$ & & $\begin{array}{l}1935 \text { Kurilo }(230) \\
1937 \text { Kurilo }(231) \\
1950 \text { Howard et al. (232) }\end{array}$ \\
\hline $117-39-5$ & $\begin{array}{r}\text { 4H-1-Benzopyran-4-one, } 2-(3,4- \\
\text { dihydroxyphenyl)-3,5,7-trihydroxy- } \\
\text { \{quercitin\} }\end{array}$ & & $\begin{array}{l}1935 \text { Kurilo (230) } \\
1936 \text { Neuberg and Kobel (233) } \\
1950 \text { Howard et al. }(232)\end{array}$ \\
\hline $153-18-4$ & $\begin{array}{c}\text { 4H-1-Benzopyran-4-one, 3-[[6-O- } \\
\text { (6-deoxy- } \alpha-L-\text {-mannopyranosyl)- } \beta \text { - } \\
D \text {-glucopyranosyl]oxy]-2-(3,4- } \\
\text { dihydroxyphenyl)-5,7-dihydroxy- } \\
\{\text { rutin\} }\end{array}$ & & $\begin{array}{l}1931 \text { Hasegawa }(234) \\
1935 \text { Kobel and Neuberg }(235) \\
1935 \text { Neuberg and Kobel }(236) \\
1936 \text { Neuberg and Kobel }(233,237) \\
1944 \text { Couch and Krewson }(238,239) \\
1944 \text { Griffith et al. }(240) \\
1947 \text { Couch }(241) \\
1949 \text { Badgett et al. }(242) \\
1951 \text { Garner }(81) \\
1951 \text { Nio and Wada (243) }\end{array}$ \\
\hline $480-10-4$ & $\begin{array}{l}\text { 4H-1-Benzopyran-4-one, 3-( } \beta-D- \\
\text { glucopyranosyloxy)-5,7- } \\
\text { dihydroxy-2-(4-hydroxyphenyl)- } \\
\text { \{kaempferol glycoside\} }\end{array}$ & & 1953 Wada (244) \\
\hline $50-32-8$ & Benzo[a]pyrene & $\begin{array}{l}1937 \text { Roffo }(245)(\text { D) } \\
1939 \text { Roffo }(70,71,72,73,178,246)(\text { D) } \\
1941 \text { Roffo }(247)(\text { D) } \\
1942 \text { Roffo }(248) \text { (D) } \\
1954 \text { Kosak (1) }\end{array}$ & \\
\hline $8001-35-2$ & $\begin{array}{l}\text { Bicyclo[2.2.1]heptane, 2,2- } \\
\text { dimethyl-3-methylene-, } \\
\text { polychlorinated } \quad\{\text { Toxaphene } \AA\}\end{array}$ & & 1951 Vinzant (60) \\
\hline $507-70-0$ & $\begin{array}{l}\text { Bicyclo[2.2.1]heptan-2-ol, 1,7,7- } \\
\text { trimethyl-, endo- } \\
\quad\{\text { borneol\} }\end{array}$ & & 1941 Sabetay et al. (90) \\
\hline $366-18-7$ & 2,2'-Bipyridine & & $\begin{array}{l}1901 \text { Pictet and Rotschy (108) } \\
1928 \text { Shmuk (140) }\end{array}$ \\
\hline $581-50-0$ & 2,3'-Bipyridine & & $\begin{array}{l}1928 \text { Shmuk (140) } \\
1936 \text { Späth and Zajic (169) } \\
1939 \text { Späth and Biniecki (249) } \\
1946 \text { Frankenburg (250) } \\
1952 \text { Frankenburg and Gottscho (97) } \\
1953 \text { Tso and Jeffrey (117) }\end{array}$ \\
\hline $581-49-7$ & $\begin{array}{l}\text { 2,3'-Bipyridine, 1,2,3,6-tetrahydro-, } \\
(\mathrm{S})-\quad\{\text {-anatabine }\}\end{array}$ & & $\begin{array}{l}1937 \text { Späth and Kesztler }(251,252) \\
1946 \text { Frankenburg }(250) \\
1948 \text { Shmuk }(253) \\
1953 \text { Tso and Jeffrey }(117)\end{array}$ \\
\hline $5953-51-5$ & $\begin{array}{l}\text { 2,3'-Bipyridine, 1,2,3,6-tetrahydro- } \\
\text { 1-methyl-, (S)- }\end{array}$ & & $\begin{array}{l}1937 \text { Späth and Kesztler (254) } \\
1946 \text { Frankenburg (250) }\end{array}$ \\
\hline $7440-69-9$ & Bismuth & & 1934 Heffer et al. (180) \\
\hline $7440-42-8$ & Boron & 1952 Bailey (118) & $\begin{array}{l}1923 \text { Warrington (255) } \\
1926 \text { Sommer and Lipman (256) } \\
1927 \text { Swanback (257) } \\
1929 \text { McMurtrey }(258) \\
1934 \text { Van Schreven (259) } \\
1935 \text { McMurtrey (199) } \\
1938 \text { McMurtrey (200) } \\
1938 \text { McMurtrey and Robinson (120) }\end{array}$ \\
\hline
\end{tabular}


Table 1. (cont.)

\begin{tabular}{|c|c|c|c|}
\hline CAS No. & Name (per CA Collective Index) & $\begin{array}{l}\text { Tobacco smoke and/or tobacco } \\
\text { distillate [dry (Dr), destructive (D)] }\end{array}$ & Tobacco \\
\hline $7440-42-8$ & Boron (cont.) & & $\begin{array}{l}1938 \text { Morgan and Street }(121) \\
1941 \text { McMurtrey }(203) \\
1950 \text { Steinberg }(260) \\
1951 \text { Garner }(81) \\
1952 \text { Lashkevvich (261) }\end{array}$ \\
\hline $123-72-8$ & Butanal & $\begin{array}{l}1908 \text { Brasch and Neuberg (64) } \\
1909 \text { Brasch }(65) \\
1931 \text { Neuberg and Burkard (66) } \\
1954 \text { Kosak (1) }\end{array}$ & \\
\hline $107-89-1$ & Butanal, 3-hydroxy- & 1931 Neuberg and Burkard (66) & \\
\hline $107-85-7$ & $\begin{array}{r}\text { 1-Butanamine, 3-methyl- } \\
\quad\{\text { isoamyl amine }\}\end{array}$ & & $\begin{array}{l}1911 \text { Ciamician and Ravenna (262) } \\
1928 \text { Shmuk (140) }\end{array}$ \\
\hline $110-15-6$ & Butanedioic acid $\quad$ \{succinic acid\} & $\begin{array}{l}1939 \text { Roffo }(70,71,72,73)(D) \\
1954 \text { Kosak (1) }\end{array}$ & $\begin{array}{l}1924 \text { Shmuk }(85) \\
1929 \text { Shmuk }(87) \\
1930 \text { Shmuk }(263)\end{array}$ \\
\hline $6915-15-7$ & $\begin{array}{l}\text { Butanedioic acid, hydroxy- } \\
\qquad\{\text { malic acid\} }\end{array}$ & & $\begin{array}{l}1809 \text { Vauquelin (264) } \\
1884 \text { Takayama (83) } \\
1894 \text { Behrens (154) } \\
1904 \text { Kissling (265) } \\
1924 \text { Shmuk (85) } \\
1929 \text { Shmuk (87) } \\
1931 \text { Yamafuji (88) } \\
1930 \text { Shmuk (263) } \\
1935 \text { Koenig (89) } \\
1937 \text { Pucher et al. (266) } \\
1939 \text { Shmuk (165) } \\
1951 \text { Bacon et al. (267) } \\
1951 \text { Garner (81) } \\
1952 \text { Bacon et al. (268) } \\
1953 \text { Phillips and Bacot (269) } \\
1953 \text { Wright and Burton (270) }\end{array}$ \\
\hline $16426-50-9$ & $\begin{array}{l}\text { Butanedioic acid, hydroxy-, } \\
\text { calcium salt }\end{array}$ & & $\begin{array}{l}1937 \text { Pucher et al. (266) } \\
1951 \text { Garner (81) }\end{array}$ \\
\hline $869-06-7$ & $\begin{array}{l}\text { Butanedioic acid, hydroxy-, } \\
\text { magnesium salt }\end{array}$ & & $\begin{array}{l}1937 \text { Pucher et al. (266) } \\
1951 \text { Garner (81) }\end{array}$ \\
\hline $585-09-1$ & $\begin{array}{l}\text { Butanedioic acid, hydroxy-, } \\
\text { potassium salt }\end{array}$ & & $\begin{array}{l}1937 \text { Pucher et al. (266) } \\
1951 \text { Garner (81) }\end{array}$ \\
\hline $431-03-8$ & 2,3-Butanedione & $\begin{array}{l}1935 \text { Neuberg and Kobel (271) } \\
1939 \text { Schmalfuss }(272) \\
1950 \text { Schmalfuss }(273) \\
1953 \text { Sasaki }(274) \\
1954 \text { Kosak (1) }\end{array}$ & $\begin{array}{l}1929 \text { Schmalfuss and Barthmeyer (275) } \\
1932 \text { Schmalfuss and Schmalfuss (276) } \\
1935 \text { Neuberg and Kobel (271) }\end{array}$ \\
\hline $107-92-6$ & Butanoic acid & $\begin{array}{l}1843 \text { Zeise }(74) \\
1871 \text { Vohl and Eulenberg }(75,76) \\
1900 \text { Thoms }(129) \\
1904 \text { Thoms }(277) \\
1931 \text { Neuberg and Burkard }(66) \\
1935 \text { Wenusch }(278) \\
1951 \text { Garner }(81) \\
1952 \text { James and Martin }(82) \\
1954 \text { Kosak (1) }\end{array}$ & $\begin{array}{l}1871 \text { Vohl and Eulenberg }(75) \\
1909 \text { Garner }(84) \\
1924 \text { Shmuk (85) } \\
1929 \text { Shmuk (87) }\end{array}$ \\
\hline $56-12-2$ & $\begin{array}{l}\text { Butanoic acid, 4-amino- } \\
\qquad\{4 \text {-aminobutyric acid }\}\end{array}$ & & $\begin{array}{l}1952 \text { Frankenburg and Gottscho (97) } \\
1953 \text { Pearse and Novellie (98) } \\
1953 \text { Zacharius and Frankenburg (99) }\end{array}$ \\
\hline $503-74-2$ & $\begin{array}{l}\text { Butanoic acid, 3-methyl- } \\
\quad\{\text { isovaleric acid\} }\end{array}$ & & 1946 Frankenburg (222) \\
\hline $2055-23-4$ & $\begin{array}{l}\text { 1-Butanone, 4-(methylamino)-1-(3- } \\
\text { pyridinyl)- } \\
\quad \text { \{pseudooxynicotine\} }\end{array}$ & & 1939 Henry (176) \\
\hline $71278-11-0$ & $\begin{array}{l}\text { 1-Butanone, 4-amino-1-(3- } \\
\text { pyridinyl)- }\end{array}$ & & 1948 Wenusch (279) \\
\hline $538-79-4$ & $\begin{array}{l}\text { 3-Buten-1-amine, } N \text {-methyl-4-(3- } \\
\text { pyridinyl)- } \\
\text { \{metanicotine; nicotimine\} }\end{array}$ & & $\begin{array}{l}1928 \text { Shmuk (140) } \\
1948 \text { Shmuk (253) }\end{array}$ \\
\hline $110-17-8$ & $\begin{array}{l}\text { 2-Butenedioic acid }(E) \text { - } \\
\text { \{fumaric acid\} }\end{array}$ & $\begin{array}{l}1939 \text { Roffo }(70,71,72,73)(D) \\
1954 \text { Kosak (1) }\end{array}$ & $\begin{array}{l}1924 \text { Shmuk (85) } \\
1929 \text { Shmuk (87) } \\
1930 \text { Shmuk (263) }\end{array}$ \\
\hline $7440-43-9$ & Cadmium & & 1934 Heffer et al. (180) \\
\hline
\end{tabular}


Table 1. (cont.)

\begin{tabular}{|c|c|c|c|}
\hline CAS No. & Name (per CA Collective Index) & $\begin{array}{l}\text { Tobacco smoke and/or tobacco } \\
\text { distillate [dry (Dr), destructive (D)] }\end{array}$ & Tobacco \\
\hline $7440-70-2$ & Calcium & 1952 Bailey (118) & $\begin{array}{l}1907 \text { Garner (280) } \\
1928 \text { Bailey and Anderson (281) } \\
1935 \text { Koenig (89) } \\
1950 \text { Steinberg }(260) \\
1951 \text { Garner }(81) \\
1952 \text { Bacon et al. (268) } \\
1953 \text { Bortner and Hamilton (282) }\end{array}$ \\
\hline $7440-44-0$ & Carbon & & $\begin{array}{l}1934 \text { Garner et al. }(283,284) \\
1951 \text { Garner }(81)\end{array}$ \\
\hline $124-38-9$ & Carbon dioxide & $\begin{array}{l}1843 \text { Zeise }(74) \\
1899 \text { Thoms }(128) \\
1900 \text { Thoms }(129) \\
1901 \text { Habermann }(285) \\
1903 / 04 \text { Moir }(286) \\
1904 \text { Habermann }(287) \\
1909 \text { Lehmann }(135) \\
1914 \text { Bush }(288) \\
1922 \text { Armstrong and Evans }(289) \\
1929 \text { Bogen }(141) \\
1929 \text { Gabelya and Kipriyanov (78) (Dr) } \\
1938 \text { Saruta }(290) \\
1938 \text { Wenusch and Schöller (291) } \\
1939 \text { De Voogd and Van der Linden (292) } \\
1939 \text { Roffo (70, 71, 72, 73) (D) } \\
1939 / 40 \text { de Campos }(293) \\
1940 \text { Haag (92) } \\
1949 \text { Fishel and Haskins (294) } \\
1952 \text { Larsen }(167) \\
1954 \text { Kosak (1) }\end{array}$ & $\begin{array}{l}1909 \text { Boekhout and de Vries (295) } \\
1934 \text { Garner et al. (283, 284) } \\
1936 \text { Dixon et al. (69) } \\
1951 \text { Garner (81) }\end{array}$ \\
\hline $75-15-0$ & Carbon disulfide & & 1951 Garner (81) \\
\hline $630-08-0$ & Carbon monoxide & 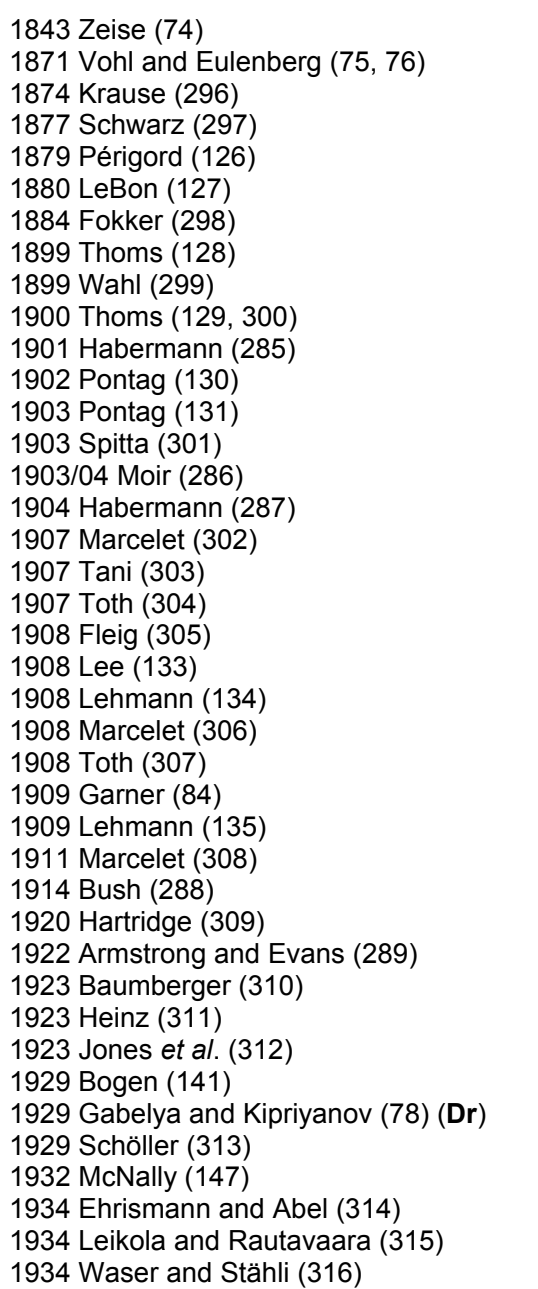 & \\
\hline
\end{tabular}


Table 1. (cont.)

\begin{tabular}{|c|c|c|c|}
\hline CAS No. & Name (per CA Collective Index) & $\begin{array}{l}\text { Tobacco smoke and/or tobacco } \\
\text { distillate [dry (Dr), destructive (D)] }\end{array}$ & Tobacco \\
\hline $630-08-0$ & Carbon monoxide (cont.) & $\begin{array}{l}1935 \text { Bastedo (187) } \\
1935 \text { Wenusch and Schöller (317) } \\
1936 \text { Bogen }(150) \\
1937 \text { Saruta }(318) \\
1937 \text { Tsumura }(319) \\
1938 \text { Roffo }(320) \\
1938 \text { Saruta }(290) \\
1938 \text { Wenusch and Schöller }(291) \\
1939 \text { De Voogd and Van der Linden (292) } \\
1939 \text { Roffo }(70,71,72,73)(D) \\
1939 / 40 \text { de Campos }(293) \\
1940 \text { Haag }(92) \\
1942 \text { Hukusima }(321) \\
1949 \text { Copenhaver and Bigelow (322) } \\
1949 \text { Fishel and Haskins (294) } \\
1951 \text { Garner }(81) \\
1951 \text { Obersteg and Scoch-Kanter (323) } \\
1954 \text { Kosak (1) }\end{array}$ & \\
\hline $463-79-6$ & Carbonic acid & & 1928 Shmuk (140) \\
\hline $10361-29-2$ & Carbonic acid, ammonium salt & 1939 Roffo $(70,71,72,73)(D)$ & \\
\hline $584-08-7$ & $\begin{array}{l}\text { Carbonic acid, dipotassium salt } \\
\text { ppotash\} }\end{array}$ & & $\begin{array}{l}1894 \text { Behrens (154) } \\
1935 \text { Koenig (89) }\end{array}$ \\
\hline $39409-82-0$ & Carbonic acid, magnesium salt & & $\begin{array}{l}1923 \text { Garner et al. }(324,325) \\
1951 \text { Garner }(81)\end{array}$ \\
\hline $7235-40-7$ & $\begin{array}{l}\beta, \beta \text {-Carotene } \\
\{\beta \text {-carotene, all-trans }\}\end{array}$ & & $\begin{array}{l}1939 \text { Nagel (326) } \\
1947 \text { Jeffrey and Griffith (327) } \\
1951 \text { Garner (81) } \\
1952 \text { Jensen (161) }\end{array}$ \\
\hline $127-40-2$ & $\begin{aligned} \beta, \varepsilon \text {-Carotene-3,3'-diol } & \\
& \{\text { xanthophyll, lutein }\}\end{aligned}$ & & $\begin{array}{l}1939 \text { Nagel }(326) \\
1951 \text { Garner }(81)\end{array}$ \\
\hline $9001-05-2$ & Catalase & & $\begin{array}{l}1936 \text { Dixon et al. }(69) \\
1937 \text { Matsumina }(171) \\
1938 \text { Hukusima }(328) \\
1946 \text { Garner }(173) \\
1951 \text { Garner }(81) \\
1951 \text { Nakai and Inaba }(174) \\
1953 \text { Brown and Steinberg (329) }\end{array}$ \\
\hline $9012-54-8$ & Cellulase & & $\begin{array}{l}1950 \text { Tracey (330) } \\
1953 \text { Barrett et al. (175) }\end{array}$ \\
\hline $9004-34-6$ & Cellulose & & $\begin{array}{l}1934 \text { Pyriki (331) } \\
1935 \text { Koenig }(89) \\
1936 \text { Brückner }(332) \\
1951 \text { Garner }(81)\end{array}$ \\
\hline $483-17-0$ & Cephalin & & 1939 Shabanov (333) \\
\hline $7440-45-1$ & Cerium & & 1950 Yamagata (334) \\
\hline $7440-46-2$ & Cesium & & $\begin{array}{l}\text { 1862/63 Grandeau (335) } \\
1913 \text { Traetta-Mosca (217) }\end{array}$ \\
\hline $16887-00-6$ & Chloride & 1954 Kosak (1) & $\begin{array}{l}1892 \text { Nessler (336) } \\
1894 \text { Behrens (154) } \\
1899 \text { Pritchard (337) } \\
1923 \text { Garner et al. (325) } \\
1925 \text { Krevs (338) } \\
1928 \text { Bailey and Anderson (281) } \\
1935 \text { Koenig (89) } \\
1941 \text { Ward (339) } \\
1950 \text { McEvoy (340) } \\
1951 \text { Garner (81) } \\
1951 \text { Mosley et al. (341) }\end{array}$ \\
\hline $479-61-8$ & Chlorophyll a & & $\begin{array}{l}1939 \text { Nagel (326) } \\
1951 \text { Garner (81) }\end{array}$ \\
\hline $519-62-0$ & Chlorophyll b & & $\begin{array}{l}1939 \text { Nagel (326) } \\
1951 \text { Garner (81) }\end{array}$ \\
\hline $1406-65-1$ & Chlorophyll $a$ and $b$ & & $\begin{array}{l}1935 \text { Koenig (89) } \\
1939 \text { Nagel (326) } \\
1950 \text { Steinberg (260) } \\
1951 \text { Bacon et al. }(267) \\
1951 \text { Garner }(81) \\
1952 \text { Bacon et al. }(268) \\
1952 \text { Jensen (161) }\end{array}$ \\
\hline
\end{tabular}


Table 1. (cont.)

\begin{tabular}{|c|c|c|c|}
\hline CAS No. & Name (per CA Collective Index) & $\begin{array}{l}\text { Tobacco smoke and/or tobacco } \\
\text { distillate [dry (Dr), destructive (D)] }\end{array}$ & Tobacco \\
\hline & Chlorophyll degradation products & $\begin{array}{l}1937 \text { Wenusch }(342) \\
1939 \text { Roffo }(70,71,72,73)(\text { D) } \\
1954 \text { Kosak }(1)\end{array}$ & \\
\hline $57-88-5$ & 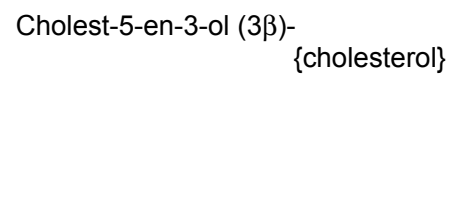 & $\begin{array}{l}1928 \text { Kennaway and Sampson (343) } \\
1938 \text { Roffo }(344) \\
1939 \text { Roffo }(345,346) \\
1941 \text { Roffo }(347) \\
1943 \text { Kirby }(348) \\
1949 \text { Falk et al. (349) }\end{array}$ & $\begin{array}{l}1928 \text { Kennaway and Sampson (343) } \\
1938 \text { Roffo (344) } \\
1939 \text { Roffo (345) } \\
1941 \text { Roffo (347) } \\
1943 \text { Kirby (348) } \\
1949 \text { Falk et al. (349) }\end{array}$ \\
\hline $7440-47-3$ & Chromium & & $\begin{array}{l}1934 \text { Heffer et al. (180) } \\
1936 \text { Johnson (350) }\end{array}$ \\
\hline $7440-48-4$ & Cobalt & & $\begin{array}{l}1934 \text { Heffer et al. }(180) \\
1952 \text { Nicholas (351) } \\
1953 \text { Nicholas and Thomas (352) }\end{array}$ \\
\hline $7440-50-8$ & Copper & 1952 Bailey (118) & $\begin{array}{l}1934 \text { Heffer et al. (180) } \\
1937 \text { Manns et al. (353) } \\
1938 \text { McMurtrey and Robinson (120) } \\
1950 \text { Swanback (354) } \\
1951 \text { Garner (81) } \\
1952 \text { Lashkevvich (261) } \\
1953 \text { Brown and Steinberg (329) }\end{array}$ \\
\hline $57-12-5$ & Cyanide & $\begin{array}{l}1932 \text { McNally (147) } \\
1954 \text { Kosak (1) }\end{array}$ & \\
\hline $608-73-1$ & $\begin{array}{l}\text { Cyclohexane, 1,2,3,4,5,6- } \\
\text { hexachloro- } \\
\quad\{\text { Lindane }(3\}\end{array}$ & & 1951 Vinzant (60) \\
\hline $327-97-9$ & 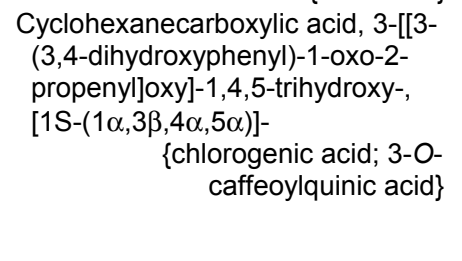 & & $\begin{array}{l}1909 \text { Gorter (355) } \\
1930 \text { Shmuk and Piatnicki (93) } \\
1933 \text { König and Dörr (356) } \\
1935 \text { Koenig (89) } \\
1939 \text { Shmuk (165) } \\
1951 \text { Garner }(81) \\
1951 \text { Roberts and Wood (96) } \\
1953 \text { Pearse and Novellie (98) }\end{array}$ \\
\hline $77-95-2$ & $\begin{array}{l}\text { Cyclohexanecarboxylic acid, } \\
\text { 1,3,4,5-tetrahydroxy- } \\
\text { \{quinic acid\} }\end{array}$ & & $\begin{array}{l}1929 \text { Shmuk }(87) \\
1930 \text { Shmuk }(357,358) \\
1939 \text { Shmuk }(165) \\
1951 \text { Garner }(81)\end{array}$ \\
\hline $89-78-1$ & $\begin{array}{l}\text { Cyclohexanol, 5-methyl-2-(1- } \\
\text { methylethyl)- } \quad \text { \{menthol\} } \\
\text { Pre-1954, considered as a } \\
\text { flavorant applied to and identified } \\
\text { on tobacco and found in its } \\
\text { smoke; subsequently identified } \\
\text { as naturally occurring in several } \\
\text { different additive-free tobaccos } \\
\text { (359) and its mechanism of } \\
\text { formation as an isoprenoid } \\
\text { defined (360) }\end{array}$ & $\begin{array}{l}1941 \text { Haggard and Greenberg (361) } \\
1952 \text { Rakieten et al. (362) }\end{array}$ & 1941 Haggard and Greenberg (361) \\
\hline $498-40-8$ & Cysteic acid & & 1953 Zacharius and Frankenburg (99) \\
\hline $52-90-4$ & $\begin{array}{l}\text { L-Cysteine } \\
\quad \text { ppropanoic acid, } 2 \text { 2-amino-3- } \\
\text { mercapto-\} }\end{array}$ & & $\begin{array}{l}1951 \text { Garner (81) } \\
1951 \text { Sharmon (363) } \\
1953 \text { Zacharius and Frankenburg (99) }\end{array}$ \\
\hline $\begin{array}{c}24645-67-8 \\
56-89-3\end{array}$ & $\begin{array}{l}\text { Cystine } \\
\quad \text { propanoic acid, 2-amino-3,3'- } \\
\text { dithiobis- }\end{array}$ & & $\begin{array}{l}1951 \text { Garner (81) } \\
1951 \text { Sharmon (363) } \\
1953 \text { Zacharius and Frankenburg (99) }\end{array}$ \\
\hline $334-48-5$ & $\begin{array}{l}\text { Decanoic acid } \quad \text { ccapric acid\} } \\
\text { Decarboxylase, } \alpha \text {-ketoglutaric acid } \\
\text { Deoxyribonucleic acid }\end{array}$ & 1952 James and Martin (82) & $\begin{array}{l}1953 \text { Barrett et al. (175) } \\
1953 \text { Silberger and Skoog (364) }\end{array}$ \\
\hline $9004-53-9$ & Dextrin & & $\begin{array}{l}1934 \text { Pyriki (331) } \\
1942 \text { Ward (172) } \\
1951 \text { Garner (81) } \\
1953 \text { Phillips and Bacot (269) }\end{array}$ \\
\hline $9000-92-4$ & Diastase & & $\begin{array}{l}1914 \text { Garner et al. }(100) \\
1942 \text { Ward (172) } \\
1951 \text { Garner (81) }\end{array}$ \\
\hline & Distillate, destructive, tobacco & $\begin{array}{l}1937 \text { Roffo }(245)(\mathbf{D}) \\
1939 \text { Roffo }(70,71,72,73)(\mathbf{D})\end{array}$ & \\
\hline
\end{tabular}




\begin{tabular}{|c|c|c|c|}
\hline CAS No. & Name (per CA Collective Index) & $\begin{array}{l}\text { Tobacco smoke and/or tobacco } \\
\text { distillate [dry (Dr), destructive (D)] }\end{array}$ & Tobacco \\
\hline & Distillate, dry, tobacco & $\begin{array}{l}1826 \text { Unterdorden (365) (Dr) } \\
1843 \text { Zeise (74) (Dr) } \\
1930 \text { Gabelya and Kipriyanov (78) (Dr) }\end{array}$ & \\
\hline \multirow[t]{3}{*}{ 143-07-7 } & Dodecanoic acid & 1952 James and Martin (82) & \\
\hline & Emulsin & & $\begin{array}{l}1913 \text { Oosthuizen and Shedd (170) } \\
1951 \text { Garner (81) }\end{array}$ \\
\hline & Enzymes, general & & $\begin{array}{l}1950 \text { Holden (366) } \\
1951 \text { Bacon et al. }(267) \\
1952 \text { Bacon et al. (268) }\end{array}$ \\
\hline $75-04-7$ & Ethanamine & 1871 Vohl and Eulenberg $(75,76)$ & 1871 Vohl and Eulenberg (75) \\
\hline $62-49-7$ & $\begin{array}{l}\text { Ethanaminium, 2-hydroxy- } N, N, N- \\
\text { trimethyl- } \\
\text { choline\} }\end{array}$ & & $\begin{array}{l}1932 \text { Nottbohm and Mayer (367) } \\
1933 \text { Vickery et al. (182) } \\
1935 \text { Vickery et al. (183) } \\
1951 \text { Garner (81) }\end{array}$ \\
\hline $460-19-5$ & Ethanedinitrile & $\begin{array}{l}1910 \text { Toth }(368,369) \\
1911 \text { Toth }(370)\end{array}$ & \\
\hline $144-62-7$ & Ethanedioic acid & 1938 Wiley et al. (371) & $\begin{array}{l}1884 \text { Takayama }(83) \\
1894 \text { Behrens }(154) \\
1904 \text { Kissling }(265) \\
1924 \text { Shmuk }(85) \\
1929 \text { Shmuk }(87) \\
1935 \text { Koenig }(89) \\
1939 \text { Shmuk (165) } \\
1951 \text { Bacon et al. }(267) \\
1952 \text { Bacon et al. }(268) \\
1953 \text { Wright and Burton (270) }\end{array}$ \\
\hline $563-72-4$ & Ethanedioic acid, calcium salt & & $\begin{array}{l}1894 \text { Behrens (154) } \\
1937 \text { Pucher et al. }(266) \\
1951 \text { Garner (81) }\end{array}$ \\
\hline $107-21-1$ & 1,2-Ethanediol $\quad\{$ ethylene glycol\} & $\begin{array}{l}1938 \text { Forbes and Haag (372) } \\
1949 \text { Reif (373) } \\
1954 \text { Kosak (1) }\end{array}$ & $\begin{array}{l}1938 \text { Wiley et al. (371) } \\
1954 \text { Kosak (1) }\end{array}$ \\
\hline $64-17-5$ & Ethanol & & 1926 Neuberg and Kobel (68) \\
\hline $111-46-6$ & $\begin{array}{l}\text { Ethanol, 2,2'-oxybis- } \\
\qquad \text { diethylene glycol\} } \\
\text { A tobacco additive applied to and } \\
\text { identified in tobacco and its } \\
\text { smoke }\end{array}$ & $\begin{array}{l}1945 \text { McNally et al. (374) } \\
1951 \text { Garner (81) } \\
1954 \text { Kosak (1) }\end{array}$ & $\begin{array}{l}1937 \text { Haag (375) } \\
1937 \text { Holck and Carlson (376) } \\
1938 \text { Newman (377) } \\
1945 \text { McNally et al. (374) } \\
1952 \text { Küchle et al. (378) } \\
1954 \text { Kosak (1) }\end{array}$ \\
\hline $350-03-8$ & $\begin{array}{l}\text { Ethanone, 1-(3-pyridinyl)- } \\
\{3 \text {-acetylpyridine; methyl 3-pyridyl } \\
\text { ketone }\}\end{array}$ & & $\begin{array}{l}1952 \text { Frankenburg and Gottscho (97) } \\
1953 \text { Tso and Jeffrey (117) }\end{array}$ \\
\hline \multirow[t]{3}{*}{$74-86-2$} & \{acetylene\} & $\begin{array}{l}1934 \text { Ehrismann and Abel (314) } \\
1949 \text { Copenhaver and Bigelow (322) } \\
1949 \text { Fishel and Haskins (294) } \\
1954 \text { Kosak (1) }\end{array}$ & \\
\hline & Fat, tobacco & & 1883 Kissling (379) \\
\hline & Fluorescent components & 1932 Hirst (380) & $\begin{array}{l}1948 \text { Best }(228) \\
1951 / 52 \text { Mizukami }(381) \\
1953 \text { Johanson }(229)\end{array}$ \\
\hline $7782-41-4$ & Fluorine & 1948 Spira (382) & \\
\hline $50-00-0$ & Formaldehyde & $\begin{array}{l}1904 \text { Thoms }(277) \\
1904 \text { Trillat }(383,384,385) \\
1905 \text { Trillat }(386) \\
1909 \text { Lehmann }(135) \\
1926 \text { Neuberg and Kobel }(68) \\
1927 \text { Neuberg and Ottenstein (387) } \\
1931 \text { Neuberg and Burkard (66) } \\
1932 \text { McNally (147) } \\
1935 \text { Bastedo (187) } \\
1954 \text { Kosak (1) }\end{array}$ & 1929 Neuberg and Kobel (388) \\
\hline $64-18-6$ & Formic acid & $\begin{array}{l}1871 \text { Vohl and Eulenberg }(75,76) \\
1929 \text { Gabelya and Kipriyanov (78) (Dr) } \\
1931 \text { Neuberg and Burkard (66) } \\
1937 \text { Bradford et al. (79) } \\
1940 \text { Haag (92) } \\
1951 \text { Garner }(81) \\
1952 \text { James and Martin (82) } \\
1954 \text { Kosak (1) }\end{array}$ & $\begin{array}{l}1871 \text { Vohl and Eulenberg (75) } \\
1924 \text { Shmuk (85) } \\
1929 \text { Shmuk (87) } \\
1930 \text { Shmuk (263) } \\
1930 \text { Shmuk and Kashirin (389) } \\
1931 \text { Neuberg and Burkard (66) } \\
1935 \text { Koenig (89) } \\
1936 \text { Dixon et al. (69) }\end{array}$ \\
\hline
\end{tabular}


Table 1. (cont.)

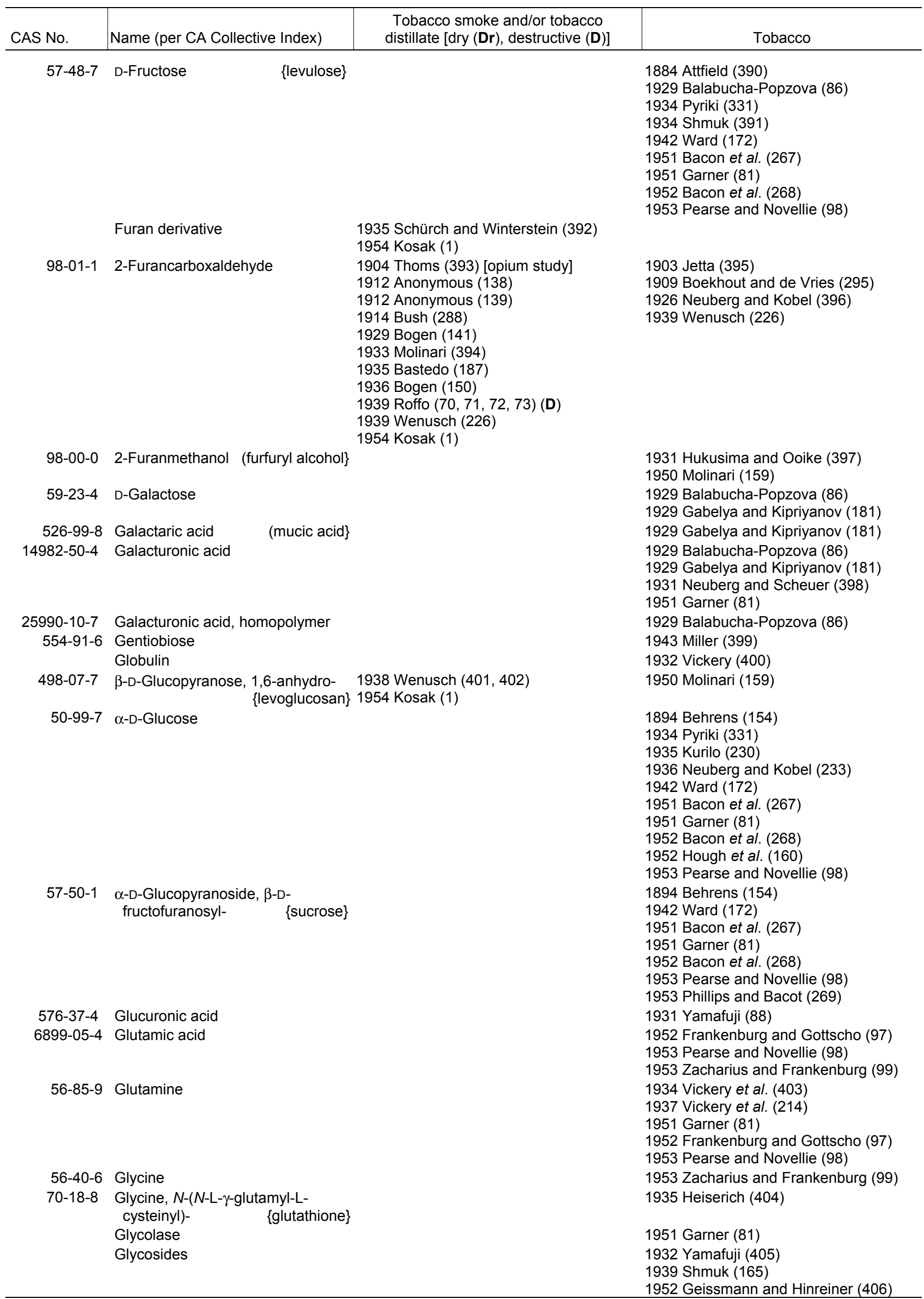


Table 1. (cont.)

\begin{tabular}{|c|c|c|c|}
\hline CAS No. & Name (per CA Collective Index) & $\begin{array}{l}\text { Tobacco smoke and/or tobacco } \\
\text { distillate [dry (Dr), destructive (D)] }\end{array}$ & Tobacco \\
\hline & $\begin{array}{l}\text { Gudham = mixture of basic } \\
\text { components of tobacco and its } \\
\text { smoke (58) }\end{array}$ & $\begin{array}{l}1935 \text { Wenusch and Schöller (56) } \\
1954 \text { Kosak (1) }\end{array}$ & 1935 Wenusch and Schöller (56) \\
\hline $9034-32-6$ & Hemicellulose & & $\begin{array}{l}1934 \text { Pyriki }(331) \\
1935 \text { Koenig }(89) \\
1951 \text { Garner }(81)\end{array}$ \\
\hline $630-04-6$ & Hentriacontane & $\begin{array}{l}1843 \text { Zeise }(74) \\
1892 \text { Abeles and Paschkis }(77) \\
1894 \text { Kissling }(407) \\
1934 \text { Wenusch (408) } \\
1935 \text { Schürch and Winterstein (392) } \\
1937 \text { Wenusch (409) } \\
1954 \text { Kosak (1) }\end{array}$ & $\begin{array}{l}1883 \text { Kissling (379) } \\
1931 \text { Hukusima and Oika (397) } \\
1934 \text { Chibnall et al. (410) } \\
1942 \text { Pyriki (411) } \\
1951 \text { Garner (81) }\end{array}$ \\
\hline $502-73-8$ & 16-Hentriacontanone & 1935 Schürch and Winterstein (392) & \\
\hline \multirow[t]{2}{*}{$593-49-7$} & Heptacosane & & $\begin{array}{l}1934 \text { Chibnall et al. (410) } \\
1931 \text { Hukusima and Oika (397) } \\
1935 \text { Shirokaya (412) } \\
1937 \text { Shmuk (413) } \\
1942 \text { Pyriki (411) } \\
1951 \text { Garner (81) }\end{array}$ \\
\hline & Heptanoic acid & 1952 James and Martin (82) & \\
\hline $123-19-3$ & $\begin{array}{l}\text { 4-Heptanone } \\
\quad \text { \{butyrone; dipropyl ketone }\}\end{array}$ & $\begin{array}{l}1931 \text { Neuberg and Burkard (66) } \\
1954 \text { Kosak (1) }\end{array}$ & 1935 Neuberg and Kobel (271) \\
\hline $57-10-3$ & Hexadecanoic acid $\quad$ palmitic acid\} & & $\begin{array}{l}1931 \text { Hukusima and Oika (397) } \\
1935 \text { Shirokaya }(412) \\
1937 \text { Salisbury }(414) \\
1937 \text { Shmuk (413) } \\
1944 \text { Venkatarao et al. (466) } \\
1945 \text { Riemenschneider et al. (227) } \\
1951 \text { Garner (81) }\end{array}$ \\
\hline $50-70-4$ & $\begin{array}{l}\text { Hexane, hexahydroxy- } \\
\text { \{sorbitol; glucitol\} }\end{array}$ & & $\begin{array}{l}1931 \text { Hasegawa }(415) \\
1936 \text { Neuberg and Kobel }(416) \\
1946 \text { Frankenburg }(222)\end{array}$ \\
\hline $142-62-1$ & Hexanoic acid & $\begin{array}{l}1871 \text { Vohl and Eulenberg }(75,76) \\
1931 \text { Neuberg and Burkard }(66) \\
1952 \text { James and Martin }(82) \\
1954 \text { Kosak (1) }\end{array}$ & . \\
\hline \multirow[t]{4}{*}{$71-00-1$} & Histidine & & $\begin{array}{l}1931 \text { Yamafuji (88) } \\
1953 \text { Pearse and Novellie (98) } \\
1953 \text { Zacharius and Frankenburg (99) }\end{array}$ \\
\hline & Hydrocarbons, aliphatic & & $\begin{array}{l}1883 \text { Kissling (379) } \\
1901 \text { Thorpe and Homes (417) }\end{array}$ \\
\hline & $\begin{array}{l}\text { Hydrocarbons, aliphatic } \mathrm{C}_{25}-\mathrm{C}_{32} \\
\text { Hydrocarbons, aromatic }\end{array}$ & $\begin{array}{l}1949 \text { Fishel and Haskins (294) } \\
1954 \text { Kosak (1) }\end{array}$ & 1883 Kissling (379) \\
\hline & Hydrocarbons, unsaturated & $\begin{array}{l}1949 \text { Fishel and Haskins (294) } \\
1954 \text { Kosak (1) }\end{array}$ & \\
\hline $74-90-8$ & $\begin{array}{l}\text { Hydrocyanic acid } \\
\qquad \text { \{hydrogen cyanide\} }\end{array}$ & $\begin{array}{l}1858 \text { Vogel and Reischauer (418) } \\
1870 \text { Poggiale and Marty }(419) \\
1871 \text { Vohl and Eulenberg (75) } \\
1880 \text { LeBon }(127) \\
1882 \text { Molnár }(420) \\
1899 \text { Thoms (128) } \\
1900 \text { Thoms (129) } \\
1901 \text { Habermann }(285) \\
1902 \text { Pontag (130) } \\
1903 \text { Habermann (421) } \\
1903 \text { Pontag (131) } \\
1903 \text { Thoms }(422) \\
1908 \text { Lee }(133) \\
1908 \text { Lehmann (134) } \\
1909 \text { Habermann and Ehrenfeld (423) } \\
1909 \text { Garner }(84) \\
1909 \text { Lehmann (135) } \\
1912 \text { Lehmann and Gundermann (424) } \\
1914 \text { Bush (288) } \\
1923 \text { Heinz (311) } \\
1934 \text { Ehrismann and Abel (314) } \\
1934 \text { Waser and Stähli (316) }\end{array}$ & $\begin{array}{l}1871 \text { Vohl and Eulenberg }(75) \\
1950 \text { Molinari (159) } \\
1951 \text { Garner }(81)\end{array}$ \\
\hline
\end{tabular}


Table 1. (cont.)

\begin{tabular}{|c|c|c|c|}
\hline CAS No. & Name (per CA Collective Index) & $\begin{array}{l}\text { Tobacco smoke and/or tobacco } \\
\text { distillate [dry (Dr), destructive (D)] }\end{array}$ & Tobacco \\
\hline 74-90-8 & $\begin{array}{l}\text { Hydrocyanic acid (cont.) } \\
\text { \{hydrogen cyanide\} }\end{array}$ & $\begin{array}{l}1938 \text { Schöller }(425,426) \\
1939 \text { Wenusch }(226) \\
1939 / 40 \text { de Campos }(293) \\
1948 \text { Haag and Larson }(427) \\
1949 \text { Fishel and Haskins }(294) \\
1951 \text { Garner }(81) \\
1954 \text { Kosak (1) }\end{array}$ & \\
\hline $1333-74-0$ & Hydrogen & 1929 Gabelya and Kipriyanov (78) (Dr) & \\
\hline \multirow[t]{3}{*}{$7783-06-4$} & Hydrogen sulfide & $\begin{array}{l}1858 \text { Vogel and Reischauer (418) } \\
1901 \text { Habermann (285) } \\
1909 \text { Garner (84) } \\
1909 \text { Habermann and Ehrenfeld (423) } \\
1909 \text { Lehmann (135) } \\
1913 \text { Toth (428) } \\
1914 \text { Bush (288) } \\
1932 \text { McNally (147) } \\
1935 \text { Wenusch (429) } \\
1938 \text { Schöller (426) } \\
1938 \text { Wenusch and Schöller (291) } \\
1939 \text { De Voogd and Van der Linden (292) } \\
1939 \text { Wenusch (226) } \\
1949 \text { Fishel and Haskins (294) } \\
1951 \text { Garner (81) } \\
1954 \text { Kosak (1) }\end{array}$ & $\begin{array}{l}1913 \text { Oosthuizen and Shedd (170) } \\
1950 \text { Molinari (159) }\end{array}$ \\
\hline & Hydropectin & & 1929 Balabucha-Popzova (86) \\
\hline & Hygroscopic agents & $\begin{array}{l}1937 \text { Ballenger and Johnson (430) } \\
1937 \text { Haag ( } 375) \\
1938 \text { Ballenger (431) } \\
1938 \text { Forbes and Haag (372) } \\
1939 \text { Ballenger (432) } \\
1940 \text { Haag (92) } \\
1945 \text { McNally et al. (374) } \\
1951 \text { Garner (81) }\end{array}$ & $\begin{array}{l}1934 \text { Mulinos and Osborne (433) } \\
1935 \text { Greenwald (434) } \\
1935 \text { Mulinos and Osborne (435) } \\
1937 \text { Holck and Carlson (376) } \\
1938 \text { Wiley et al. }(371) \\
1952 \text { Küchle et al. }(378,436)\end{array}$ \\
\hline $6917-35-7$ & Inositol & & $\begin{array}{l}1930 \text { Shmuk }(263,358) \\
1935 \text { Neuberg and Kobel }(271) \\
1951 \text { Garner }(81)\end{array}$ \\
\hline $3615-82-5$ & $\begin{array}{l}\text { myo-Inositol, hexakis(dihydrogen } \\
\text { phosphate), calcium magnesium } \\
\text { salt }\end{array}$ & & 1930 Shmuk (263) \\
\hline & Inulase & & 1913 Oosthuizen and Shedd (170) \\
\hline $9001-57-4$ & Invertase & & $\begin{array}{l}1913 \text { Oosthuizen and Shedd (170) } \\
1936 \text { Dixon et al. (69) } \\
1937 \text { Matsumina (171) } \\
1946 \text { Garner (173) } \\
1951 \text { Garner (81) } \\
1951 \text { Nakai and Inaba (174) } \\
1953 \text { Barrett et al. (175) }\end{array}$ \\
\hline $7553-56-2$ & lodine & & $\begin{array}{l}1930 \text { Schwaibold (437) } \\
1935 \text { McMurtrey (199) } \\
1938 \text { McMurtrey (200) } \\
1941 \text { McMurtrey (203) } \\
1953 \text { Wolff et al. (208) }\end{array}$ \\
\hline $7439-89-6$ & Iron & 1952 Bailey (118) & $\begin{array}{l}1888 \text { Fesca and Imai (438) } \\
1909 \text { Boekhout and de Vries (295) } \\
1929 \text { Coolhaas (439) } \\
1933 \text { Starkenstein and Stejskal (440) } \\
1937 \text { McMurtrey (441) } \\
1938 \text { McMurtrey and Robinson (120) } \\
1946 \text { LeCompte (442) } \\
1951 \text { Garner (81) } \\
1953 \text { Brown and Steinberg (329) }\end{array}$ \\
\hline \multirow[t]{3}{*}{ 7004-09-3 } & Isoleucine & & $\begin{array}{l}1950 \text { Steinberg (260) } \\
1953 \text { Zacharius and Frankenburg (99) }\end{array}$ \\
\hline & Isonicoteine & & 1914 Noga (443) \\
\hline & Lactase & & $\begin{array}{l}1913 \text { Oosthuizen and Shedd (170) } \\
1946 \text { Garner (173) } \\
1953 \text { Barrett et al. (175) }\end{array}$ \\
\hline \multirow[t]{2}{*}{$63-42-3$} & Lactose & & 1952 Hough et al. (160) \\
\hline & $\begin{array}{l}\text { Lathrein = a mixture of basic } \\
\text { components of tobacco and its } \\
\text { smoke }(58)\end{array}$ & $\begin{array}{l}1935 \text { Wenusch and Schöller (55) } \\
1936 \text { Wenusch and Schöller (177) } \\
1954 \text { Kosak (1) }\end{array}$ & 1935 Wenusch and Schöller (55) \\
\hline
\end{tabular}


Table 1. (cont.)

\begin{tabular}{|c|c|c|c|}
\hline CAS No. & Name (per CA Collective Index) & $\begin{array}{l}\text { Tobacco smoke and/or tobacco } \\
\text { distillate [dry (Dr), destructive (D)] }\end{array}$ & Tobacco \\
\hline $7439-92-1$ & Lead & 1952 Bailey (118) & $\begin{array}{l}1861 \text { Creegan (444) } \\
1921 \text { Caddenhead and Jacques (445) } \\
1927 \text { Prat }(446) \\
1929 / 30 \text { Reitzel }(447) \\
1934 \text { Heffer et al. (180) } \\
1952 \text { Bunce (207) }\end{array}$ \\
\hline $8002-43-5$ & Lecithins & & $\begin{array}{l}1894 \text { Behrens (154) } \\
1937 \text { Shabanov (448) }\end{array}$ \\
\hline $7005-03-0$ & Leucine & & $\begin{array}{l}1953 \text { Pearse and Novellie (98) } \\
1953 \text { Zacharius and Frankenburg (99) }\end{array}$ \\
\hline $9005-53-2$ & Lignin & & $\begin{array}{l}1934 \text { Pyriki }(331 \\
1951 \text { Garner }(81) \\
1953 \text { Phillips and Bacot }(269)\end{array}$ \\
\hline $9001-62-1$ & Lipase & & $\begin{array}{l}1913 \text { Oosthuizen and Shedd (170) } \\
1946 \text { Garner (173) } \\
1951 \text { Garner }(81)\end{array}$ \\
\hline $7439-93-2$ & Lithium & & $\begin{array}{l}1909 \text { Ravenna and Zamorani (449) } \\
1913 \text { Traetta-Mosca }(217) \\
1921 \text { Headden }(220) \\
1935 \text { McMurtrey }(199) \\
1938 \text { McMurtrey }(200) \\
1941 \text { McMurtrey (203) } \\
1950 \text { Yamagata }(334) \\
1951 \text { Garner (81) }\end{array}$ \\
\hline & $\begin{array}{l}\text { Lohitam }=\text { mixture of basic } \\
\text { components in tobacco and its } \\
\text { smoke (58) }\end{array}$ & $\begin{array}{l}1935 \text { Wenusch and Schöller (55) } \\
1936 \text { Wenusch and Schöller (177) } \\
1954 \text { Kosak (1) }\end{array}$ & 1935 Wenusch and Schöller (55) \\
\hline $6899-06-5$ & Lysine & & $\begin{array}{l}1953 \text { Pearse and Novellie (98) } \\
1953 \text { Zacharius and Frankenburg (99) }\end{array}$ \\
\hline $7439-95-4$ & Magnesium & 1952 Bailey (118) & $\begin{array}{l}1922 \text { Garner et al. (450) } \\
1923 \text { Garner et al. (325) } \\
1928 \text { Bailey and Anderson (281) } \\
1931 \text { Anderson et al. (451) } \\
1935 \text { Koenig (89) } \\
1936 \text { Anderson et al. (452) } \\
1937 \text { McMurtrey (441) } \\
1941 \text { Ward (339) } \\
1950 \text { Steinberg (260) } \\
1951 \text { Garner (81) } \\
1953 \text { Bortner and Hamilton (282) }\end{array}$ \\
\hline $69-79-4$ & $\alpha$-Maltose & & $\begin{array}{l}1934 \text { Pyriki (331) } \\
1941 \text { Pyriki (453) } \\
1942 \text { Ward (172) } \\
1951 \text { Garner (81) } \\
1952 \text { Hough et al. (160) }\end{array}$ \\
\hline $7439-96-5$ & Manganese & 1952 Bailey (118) & $\begin{array}{l}1910 \text { Mach (454) } \\
1934 \text { Heffer et al. (180) } \\
1938 \text { McMurtrey and Robinson (120) } \\
1941 \text { Ward (339) } \\
1951 \text { Garner (81) } \\
1953 \text { Brown and Steinberg (329) }\end{array}$ \\
\hline $3615-41-6$ & $\begin{array}{l}\text { L-Mannose, 6-deoxy- } \\
\{\alpha \text {-rhamnose }\}\end{array}$ & & 1929 Shmuk (87) \\
\hline $8049-97-6$ & Melanin & & 1934 Shmuk and Shirokaya (455) \\
\hline $7439-97-6$ & Mercury & & $\begin{array}{l}1934 \text { Heffer et al. (180) } \\
1936 \text { Kincaid (456) }\end{array}$ \\
\hline $74-89-5$ & $\{$ methylamine $\}$ & $\begin{array}{l}1929 \text { Gabelya and Kipriyanov (78) (Dr) } \\
1954 \text { Kosak (1) }\end{array}$ & 1928 Shmuk (140) \\
\hline $75-50-3$ & $\begin{array}{l}\text { Methanamine, } \mathrm{N}, \mathrm{N} \text {-dimethyl- } \\
\text { \{trimethylamine\} }\end{array}$ & 1929 Gabelya and Kipriyanov (78) (Dr) & $\begin{array}{l}1929 \text { Gabelya and Kipriyanov }(78,181) \\
1936 \text { Späth and Zajic }(169) \\
1939 \text { Späth and Biniecki }(249)\end{array}$ \\
\hline $124-40-3$ & $\begin{aligned} \text { Methanamine, } \mathrm{N} \text {-methyl- } \\
\\
\text { \{dimethylamine\} }\end{aligned}$ & 1929 Gabelya and Kipriyanov (78) (Dr) & 1928 Shmuk (140) \\
\hline $107-43-7$ & $\begin{array}{c}\text { Methanaminium, } 1 \text {-carboxy- } N, N, N- \\
\text { trimethyl-, inner salt } \quad\{\text { betaine }\}\end{array}$ & & $\begin{array}{l}1928 \text { Shmuk (140) } \\
1933 \text { Vickery et al. (182) } \\
1935 \text { Vickery et al. (183) } \\
1934 \text { Nito and Kitamura (221) } \\
1951 \text { Garner (81) } \\
1952 \text { Willaman (457) }\end{array}$ \\
\hline
\end{tabular}




\begin{tabular}{|c|c|c|c|}
\hline CAS No. & Name (per CA Collective Index) & $\begin{array}{l}\text { Tobacco smoke and/or tobacco } \\
\text { distillate [dry (Dr), destructive (D)] }\end{array}$ & Tobacco \\
\hline $74-82-8$ & Methane & $\begin{array}{l}1871 \text { Vohl and Eulenberg }(75,76) \\
1909 \text { Lehmann }(135)\end{array}$ & \\
\hline $67-56-1$ & Methanol & $\begin{array}{l}1909 \text { Lehmann (135) } \\
1927 \text { Neuberg and Ottenstein (387) } \\
1929 \text { Neuberg and Kobel (388) } \\
1929 \text { Shmuk (458) } \\
1931 \text { Neuberg and Burkard (66) } \\
1932 \text { McNally }(147) \\
1939 \text { Roffo }(70,71,72,73)(D) \\
1939 \text { Wenusch }(226) \\
1951 \text { Garner }(81) \\
1954 \text { Kosak (1) }\end{array}$ & $\begin{array}{l}1926 \text { Neuberg and Kobel (68) } \\
1929 \text { Balabucha-Popzova (86) } \\
1929 \text { Neuberg and Kobel (388) } \\
1929 \text { Shmuk (458) } \\
1931 \text { Neuberg and Burkard (66) } \\
1931 \text { Neuberg and Scheuer (398) } \\
1936 \text { Lokschina (459) } \\
1939 \text { Shmuk (165) } \\
1951 \text { Garner (81) }\end{array}$ \\
\hline $63-68-3$ & L-Methionine & & $\begin{array}{l}1951 \text { Sharmon (363) } \\
1953 \text { Zacharius and Frankenburg (99) }\end{array}$ \\
\hline $7439-98-7$ & Molybdenum & & $\begin{array}{l}1939 \text { Arnon and Stout (460) } \\
1947 \text { Kozlowska (461) } \\
1953 \text { Brown and Steinberg (329) }\end{array}$ \\
\hline $9047-56-7$ & Mutase & & 1926 Neuberg and Kobel (68) \\
\hline $9033-12-9$ & Mutase, ketone-aldehyde & & 1951 Garner (81) \\
\hline $7440-02-0$ & Nickel & 1952 Bailey (118) & $\begin{array}{l}1934 \text { Heffer et al. (180) } \\
1952 \text { Nicholas ( } 351) \\
1952 \text { Hunter and Vergnano (462) } \\
1952 \text { Shira and Kodaira (463) }\end{array}$ \\
\hline & $\begin{array}{l}\text { Nicoteine }=\text { nornicotine }+ \\
\text { anatabine }\end{array}$ & & $\begin{array}{l}1914 \text { Noga (443) } \\
1928 \text { Ehrenstein (111) } \\
1928 \text { Shmuk (140) } \\
1935 \text { Späth and Zajic (464) } \\
1936 \text { Späth et al. (465) } \\
1939 \text { Henry (176) }\end{array}$ \\
\hline & Nicotoine & & 1914 Noga (443) \\
\hline $14797-55-8$ & Nitrate & $\begin{array}{l}1932 \text { McNally (147) } \\
1954 \text { Kosak (1) }\end{array}$ & $\begin{array}{l}1894 \text { Behrens (154) } \\
1951 \text { Garner (81) }\end{array}$ \\
\hline $7697-37-2$ & Nitric acid & & $\begin{array}{l}1885 \text { Müller-Thurgen (153) } \\
1894 \text { Behrens (154) }\end{array}$ \\
\hline $7757-79-1$ & Nitric acid, potassium salt & & 1894 Behrens (154) \\
\hline $7727-37-9$ & Nitrogen & 1877 Schwarz (297) & $\begin{array}{l}1934 \text { Garner et al. }(283,284) \\
1951 \text { Garner }(81)\end{array}$ \\
\hline \multirow[t]{3}{*}{$630-03-5$} & Nonacosane & & 1934 Chibnall et al. (410) \\
\hline & Nonanoic acid & 1952 James and Martin (82) & \\
\hline & Obelin = ammonia (58) & $\begin{array}{l}1935 \text { Wenusch and Schöller }(55,56) \\
1936 \text { Wenusch and Schöller }(177) \\
1954 \text { Kosak (1) }\end{array}$ & $\begin{array}{l}1928 \text { Shmuk (140) } \\
1929 \text { Gabelya and Kipriyanov }(78) \\
1935 \text { Wenusch and Schöller }(55,56) \\
1950 \text { Molinari }(159)\end{array}$ \\
\hline $506-21-8$ & $\begin{array}{l}\text { 9,12-Octadecadienoic acid } \\
\qquad\{\text { linoleic acid\} }\end{array}$ & & $\begin{array}{l}1937 \text { Salisbury (414) } \\
1937 \text { Shmuk (413) } \\
1944 \text { Venkatarao et al. (466) } \\
1945 \text { Riemenschneider et al. (227) } \\
1951 \text { Garner (81) }\end{array}$ \\
\hline $57-11-4$ & Octadecanoic acid $\quad\{$ stearic acid $\}$ & & $\begin{array}{l}1937 \text { Salisbury (414) } \\
1944 \text { Venkatarao et al. (466) } \\
1945 \text { Riemenschneider et al. (227) } \\
1951 \text { Garner (81) }\end{array}$ \\
\hline $463-40-1$ & $\begin{array}{r}9,12,15-\text { Octadecatrienoic acid } \\
\quad\{\text { linolenic acid\} }\end{array}$ & & $\begin{array}{l}1935 \text { Shirokaya (412) } \\
1944 \text { Venkatarao et al. (466) }\end{array}$ \\
\hline $112-80-1$ & 9-Octadecenoic acid $\quad\{$ oleic acid\} & & $\begin{array}{l}1937 \text { Salisbury (414) } \\
1937 \text { Shmuk (413) } \\
1944 \text { Venkatarao et al. (466) } \\
1945 \text { Riemenschneider et al. (227) } \\
1951 \text { Garner (81) }\end{array}$ \\
\hline $78-70-6$ & $\begin{array}{r}\text { 1,6-Octadien-3-ol, 3,7-dimethyl- } \\
\quad\{\text { linalool }\}\end{array}$ & & 1941 Sabetay et al. (90) \\
\hline $124-07-2$ & Octanoic acid $\quad\{$ caprylic acid $\}$ & $\begin{array}{l}1871 \text { Vohl and Eulenberg }(75,76) \\
1952 \text { James and Martin }(82)\end{array}$ & $\begin{array}{l}1941 \text { Sabetay et al. (467) } \\
1946 \text { Frankenburg (222) }\end{array}$ \\
\hline $372-75-8$ & $\begin{array}{r}\text { L-Ornithine, N5-(aminocarbonyl)- } \\
\text { \{citrulline\} }\end{array}$ & & 1953 Zacharius and Frankenburg (99) \\
\hline $9035-73-8$ & Oxidase & & $\begin{array}{l}1910 \text { Betting (468) } \\
1913 \text { Oosthuizen and Shedd (170) } \\
1928 \text { Shmuk (140) }\end{array}$ \\
\hline
\end{tabular}




\begin{tabular}{|c|c|c|c|}
\hline CAS No. & Name (per CA Collective Index) & $\begin{array}{l}\text { Tobacco smoke and/or tobacco } \\
\text { distillate [dry (Dr), destructive (D)] }\end{array}$ & Tobacco \\
\hline $9035-73-8$ & Oxidase (cont.) & & $\begin{array}{l}1941 \text { Roberts }(469) \\
1946 \text { Garner }(173) \\
1951 \text { Garner }(81) \\
1953 \text { Barrett et al. }(175)\end{array}$ \\
\hline $9029-44-1$ & Oxidase, ascorbate & & 1953 Brown and Steinberg (329) \\
\hline $9001-16-5$ & Oxidase, cytochrome c & & 1953 Barrett et al. (175) \\
\hline $7782-44-7$ & Oxygen & 1954 Kosak (1) & 1950 Molinari (159) \\
\hline $79-83-4$ & Pantothenic acid & & 1952 Jensen (161) \\
\hline & Paraffins & 1893 Kissling (223) & 1901 Thorpe and Homes (417) \\
\hline $9025-98-3$ & Pectase & & $\begin{array}{l}1946 \text { Garner (173) } \\
1951 \text { Garner (81) }\end{array}$ \\
\hline $9046-40-6$ & Pectic acid & & $\begin{array}{l}1884 \text { Takayama }(83) \\
1929 \text { Balabucha-Popzova }(86) \\
1931 \text { Neuberg and Scheuer (398) } \\
1951 \text { Garner }(81) \\
1953 \text { Phillips and Bacot }(269)\end{array}$ \\
\hline $9000-69-5$ & Pectin & & $\begin{array}{l}1903 \text { Jetta (395) } \\
1929 \text { Balabucha-Popzova (86) } \\
1929 \text { Gabelya and Kipriyanov (181) } \\
1931 \text { Neuberg and Scheuer (398) } \\
1934 \text { Pyriki (331) } \\
1935 \text { Koenig (89) } \\
1951 \text { Garner (81) } \\
1953 \text { Phillips and Bacot (269) }\end{array}$ \\
\hline & $\begin{array}{l}\text { Pectinic acid } \\
\text { I-Peganin }\end{array}$ & & $\begin{array}{l}1929 \text { Gabelya and Kipriyanov (181) } \\
1936 \text { Späth and Zajic (169) }\end{array}$ \\
\hline $109-52-4$ & Pentanoic acid & $\begin{array}{l}1871 \text { Vohl and Eulenberg }(75,76) \\
1929 \text { Gabelya and Kipriyanov }(78)(\mathrm{Dr}) \\
1931 \text { Neuberg and Burkard }(66) \\
1952 \text { James and Martin }(82) \\
1954 \text { Kosak (1) }\end{array}$ & $\begin{array}{l}1871 \text { Vohl and Eulenberg (75) } \\
1929 \text { Gabelya and Kipriyanov (78) } \\
1947 \text { Sabetay and Panouse (470) }\end{array}$ \\
\hline $105-43-1$ & Pentanoic acid, 3-methyl- & & 1947 Sabetay and Panouse (470) \\
\hline $96-22-0$ & 3-Pentanone & $\begin{array}{l}1927 \text { Neuberg and Ottenstein (387) } \\
1931 \text { Neuberg and Burkard (66) } \\
1954 \text { Kosak (1) }\end{array}$ & 1935 Neuberg and Kobel (271) \\
\hline $116001-96-8$ & Pentosan & & $\begin{array}{l}1903 \text { Jetta (395) } \\
1914 \text { Garner et al. (100) } \\
1935 \text { Koenig }(89) \\
1953 \text { Phillips and Bacot }(269)\end{array}$ \\
\hline & Pentose & & $\begin{array}{l}1931 \text { Neuberg and Scheuer (398) } \\
1934 \text { Pyriki (331) } \\
1951 \text { Garner (81) }\end{array}$ \\
\hline & Pentosenucleic acid & & 1953 Silberger and Skoog (364) \\
\hline & Peptide & & $\begin{array}{l}1937 \text { Vickery et al. (214) } \\
1951 \text { Garner (81) }\end{array}$ \\
\hline $9003-99-0$ & Peroxidase & & $\begin{array}{l}1910 \text { Betting (468) } \\
1937 \text { Matsumina (171) } \\
1951 \text { Garner }(81) \\
1951 \text { Nakai and Inaba (174) } \\
1953 \text { Barrett et al. (175) } \\
1953 \text { Brown and Steinberg (329) }\end{array}$ \\
\hline \multirow[t]{2}{*}{$85-01-8$} & Phenanthrene & $\begin{array}{l}1939 \text { Roffo }(70,71,72,73)(D) \\
1954 \text { Kosak (1) }\end{array}$ & \\
\hline & Phenanthrene derivative & 1939 Roffo (345) & \\
\hline $108-95-2$ & Phenol & $\begin{array}{l}1871 \text { Vohl and Eulenberg }(75,76) \\
1914 \text { Bush }(288) \\
1936 \text { Bogen }(150) \\
1936 \text { Molinari }(224) \\
1940 \text { Haag }(92) \\
1947 \text { Ikeda }(215) \\
1952 \text { Rayburn }(471) \\
1953 \text { Rayburn et al. }(472) \\
1954 \text { Kosak }(1)\end{array}$ & $\begin{array}{l}1871 \text { Vohl and Eulenberg }(75) \\
1939 \text { Wenusch }(226) \\
1950 \text { Molinari }(159) \\
1953 \text { Rayburn et al. (472) }\end{array}$ \\
\hline $90-05-1$ & Phenol, 2-methoxy- & $\begin{array}{l}1952 \text { Rayburn (471) } \\
1953 \text { Rayburn et al. (472) }\end{array}$ & $\begin{array}{l}1939 \text { Wenusch (226) } \\
1953 \text { Rayburn et al. (472) }\end{array}$ \\
\hline $97-54-1$ & $\begin{array}{r}\text { Phenol, 2-methoxy-4-(1-propenyl)- } \\
\text { \{isoeugenol\} }\end{array}$ & & 1946 Frankenburg (222) \\
\hline
\end{tabular}


Table 1. (cont.)

\begin{tabular}{|c|c|c|c|}
\hline CAS No. & Name (per CA Collective Index) & $\begin{array}{l}\text { Tobacco smoke and/or tobacco } \\
\text { distillate [dry (Dr), destructive (D)] }\end{array}$ & Tobacco \\
\hline $97-53-0$ & \multicolumn{2}{|l|}{$\begin{array}{r}\text { Phenol, 2-methoxy-4-(2-propenyl)- } \\
\text { \{eugenol\} }\end{array}$} & $\begin{array}{l}1941 \text { Sabetay et al. (467) } \\
1946 \text { Frankenburg (222) }\end{array}$ \\
\hline $1319-77-3$ & Phenol, methyl- & 1936 Bogen (150) & \\
\hline $95-48-7$ & Phenol, 2-methyl- & $\begin{array}{l}1952 \text { Rayburn (471) } \\
1953 \text { Rayburn et al. (472) }\end{array}$ & \\
\hline $108-39-4$ & Phenol, 3-methyl- & $\begin{array}{l}1952 \text { Rayburn (471) } \\
1953 \text { Rayburn et al. (472) }\end{array}$ & 1953 Rayburn et al. (472) \\
\hline \multirow[t]{2}{*}{$106-44-5$} & Phenol, 4-methyl- & & \\
\hline & Phenols, structure unspecified & 1954 Kosak (1) & $\begin{array}{l}1934 \text { Shmuk (391) } \\
1934 \text { Shmuk and Shirokaya (455) }\end{array}$ \\
\hline $63-91-2$ & Phenylalanine & & $\begin{array}{l}1953 \text { Pearse and Novellie (98) } \\
1953 \text { Zacharius and Frankenburg (99) }\end{array}$ \\
\hline \multirow[t]{2}{*}{$14265-44-2$} & Phosphate & & $\begin{array}{l}1894 \text { Behrens (154) } \\
1935 \text { Koenig (89) }\end{array}$ \\
\hline & Phosphatase & & $\begin{array}{l}1946 \text { Garner (173) } \\
1951 \text { Garner }(81)\end{array}$ \\
\hline $7803-51-2$ & Phosphine & 1898 Cavalli (473) & \\
\hline $56-38-2$ & $\begin{array}{l}\text { Phosphorothioic acid, O, O-diethyl } \\
\text { O-(4-nitrophenyl) ester } \\
\qquad\{\text { Parathion } \circledast\}\end{array}$ & & 1951 Vinzant (60) \\
\hline \multirow[t]{3}{*}{$7723-14-0$} & Phosphorus & & $\begin{array}{l}1928 \text { Bailey and Anderson (281) } \\
1941 \text { Ward }(339) \\
1950 \text { Steinberg }(260) \\
1951 \text { Garner }(81) \\
1953 \text { Bortner and Hamilton (282) }\end{array}$ \\
\hline & Phytosterol & 1954 Kosak (1) & $\begin{array}{l}1913 \text { Traetta-Mosca (474) } \\
1935 \text { Kobel and Neuberg }(235) \\
1935 \text { Schürch and Winterstein (392) } \\
1937 \text { Shmuk (413) }\end{array}$ \\
\hline & Pigments & & $\begin{array}{l}1935 \text { Koenig (89) } \\
1939 \text { Nagel }(326)\end{array}$ \\
\hline $110-89-4$ & Piperidine & & 1936 Späth and Zajic (169) \\
\hline \multirow[t]{2}{*}{$535-75-1$} & $\begin{array}{l}\text { 2-Piperidinecarboxylic acid } \\
\text { \{pipecolic acid\} }\end{array}$ & & 1953 Zacharius and Frankenburg (99) \\
\hline & $\begin{array}{l}\text { Poikiline = 1-Butanone, 4-amino-l- } \\
\text { (3-pyridyl)- }\end{array}$ & 1954 Kosak (1) & 1948 Wenusch $(279)$ \\
\hline $7440-09-7$ & Potassium & 1952 Bailey (118) & $\begin{array}{l}1923 \text { Garner et al. (325) } \\
1928 \text { Bailey and Anderson (281) } \\
1950 \text { Steinberg (260) } \\
1951 \text { Garner (81) } \\
1953 \text { Bortner and Hamilton (282) } \\
1953 \text { Wolff et al. (208) }\end{array}$ \\
\hline $147-85-3$ & Proline & & $\begin{array}{l}1952 \text { Frankenburg and Gottscho (97) } \\
1953 \text { Pearse and Novellie (98) } \\
1953 \text { Zacharius and Frankenburg (99) }\end{array}$ \\
\hline $78-98-8$ & $\begin{array}{l}\text { Propanal, 2-oxo- } \\
\quad \text { ppyruvaldehyde; methylglyoxal\} }\end{array}$ & & 1929 Kobel and Scheuer (475) \\
\hline $141-82-2$ & Propanedioic acid $\quad$ \{malonic acid\} & & 1953 Wada and Kobashi (94) \\
\hline $77-92-9$ & $\begin{array}{l}\text { 1,2,3-Propanetricarboxylic acid, } 2- \\
\text { hydroxy- } \\
\text { \{citric acid\} }\end{array}$ & 1939 Roffo $(70,71,72,73)(D)$ & $\begin{array}{l}1884 \text { Takayama (83) } \\
1894 \text { Behrens (154) } \\
1904 \text { Kissling (265) } \\
1924 \text { Shmuk (85) } \\
1930 \text { Shmuk (357) } \\
1930 \text { Shmuk and Piatnicki (93) } \\
1931 \text { Yamafuji (88) } \\
1935 \text { Koenig (89) } \\
1939 \text { Shmuk (165) } \\
1951 \text { Bacon et al. (267) } \\
1952 \text { Bacon et al. (268) } \\
1953 \text { Phillips and Bacot (269) } \\
1953 \text { Wright and Burton (270) }\end{array}$ \\
\hline $813-94-5$ & $\begin{array}{l}\text { 1,2,3-Propanetricarboxylic acid, } 2- \\
\text { hydroxy-, calcium salt }\end{array}$ & & $\begin{array}{l}1937 \text { Pucher et al. (266) } \\
1951 \text { Garner (81) }\end{array}$ \\
\hline $3344-18-1$ & $\begin{array}{l}\text { 1,2,3-Propanetricarboxylic acid, 2- } \\
\text { hydroxy-, magnesium salt }\end{array}$ & & $\begin{array}{l}1937 \text { Pucher et al. (266) } \\
1951 \text { Garner (81) }\end{array}$ \\
\hline $6100-05-6$ & $\begin{array}{l}\text { 1,2,3-Propanetricarboxylic acid, } 2- \\
\text { hydroxy-, potassium salt }\end{array}$ & & $\begin{array}{l}1937 \text { Pucher et al. (266) } \\
1951 \text { Garner (81) }\end{array}$ \\
\hline
\end{tabular}


Table 1. (cont.)

\begin{tabular}{|c|c|c|c|}
\hline CAS No. & Name (per CA Collective Index) & $\begin{array}{l}\text { Tobacco smoke and/or tobacco } \\
\text { distillate [dry (Dr), destructive (D)] }\end{array}$ & Tobacco \\
\hline $56-81-5$ & $\begin{array}{l}\text { 1,2,3-Propanetriol }\{\text { glycerol\} } \\
\text { A tobacco additive applied to and } \\
\text { identified in tobacco and its } \\
\text { smoke; in 1964, it was identified } \\
\text { as a component of additive-free, } \\
\text { uncased tobacco (478) }\end{array}$ & $\begin{array}{l}1938 \text { Forbes and Haag (372) } \\
1945 \text { McNally et al. (374) } \\
1951 \text { Garner (81) } \\
1954 \text { Kosak (1) }\end{array}$ & $\begin{array}{l}1937 \text { Holck and Carlson (376) } \\
1937 \text { Shmuk (413) } \\
1945 \text { McNally et al. (374) } \\
1946 \text { Frankenburg (222) }\end{array}$ \\
\hline $79-09-4$ & Propanoic acid $\quad$ \{propionic acid\} & $\begin{array}{l}1871 \text { Vohl and Eulenberg }(75,76) \\
1929 \text { Gabelya and Kipriyanov (78) (Dr) } \\
1952 \text { James and Martin (82) }\end{array}$ & $\begin{array}{l}1924 \text { Shmuk (85) } \\
1929 \text { Gabelya and Kipriyanov (78) } \\
1929 \text { Shmuk (87) }\end{array}$ \\
\hline \multirow[t]{2}{*}{$50-21-5$} & $\begin{array}{l}\text { Propanoic acid, 2-hydroxy- } \\
\qquad\{\text { lactic acid\} }\end{array}$ & & 1953 Wada and Kobashi (94) \\
\hline & $\begin{aligned} \text { 1-Propanone, } & 1-(3-p y r i d i n y l)- \\
& \{\text { pyridyl ethyl ketone }\end{aligned}$ & $\begin{array}{l}1919 \text { Kissling }(477) \\
1935 \text { Schöller }(478) \\
1939 \text { Wenusch }(226) \\
1954 \text { Kosak }(1)\end{array}$ & 1928 Shmuk (140) \\
\hline $67-64-1$ & 2-Propanone & 1937 Wenusch (409) & 1871 Vohl and Eulenberg (75) \\
\hline $107-02-8$ & 2-Propenal & $\begin{array}{l}1932 \text { McNally }(147) \\
1936 \text { Bogen }(150) \\
1937 \text { Ribeiro }(479) \\
1954 \text { Kosak (1) }\end{array}$ & \\
\hline $331-39-5$ & $\begin{array}{l}\text { 2-Propenoic acid, 3-(3,4- } \\
\text { dihydroxyphenyl)- } \quad \text { \{caffeic acid\} }\end{array}$ & 1939 Shmuk (165) & $\begin{array}{l}1894 \text { Behrens (154) } \\
1929 \text { Shmuk }(87) \\
1930 \text { Shmuk }(357,480) \\
1930 \text { Shmuk and Piatnicki (93) } \\
1935 \text { Koenig (89) } \\
1939 \text { Shmuk (165) } \\
1951 \text { Garner (81) }\end{array}$ \\
\hline \multirow[t]{2}{*}{$78990-62-2$} & Protease & & $\begin{array}{l}1913 \text { Oosthuizen and Shedd (170) } \\
1951 \text { Garner (81) }\end{array}$ \\
\hline & Protein & & $\begin{array}{l}1886 \text { Mendel (481) } \\
1894 \text { Behrens (154) } \\
1930 \text { Smirnov and Izvoshtshikov (155) } \\
1933 \text { Vickery et al. (182) } \\
1935 \text { Koenig (89) } \\
1935 \text { Vickery et al. (183) } \\
1951 \text { Garner (81) }\end{array}$ \\
\hline $20-73-0$ & $1 H$-Purine & & 1950 Steinberg $(260)$ \\
\hline $73-24-5$ & $1 H$-Purin-6-amine & & $\begin{array}{l}1931 \text { Yamafuji (88) } \\
1933 \text { Vickery et al. (182) } \\
1935 \text { Vickery et al. (183) } \\
1934 \text { Nito and Kitamura (221) } \\
1951 \text { Garner (81) }\end{array}$ \\
\hline $73-40-5$ & $\begin{array}{l}\text { 6H-Purin-6-one, 2-amino-1,7- } \\
\text { dihydro- }\end{array}$ & & $\begin{array}{l}1935 \text { Vickery et al. (183) } \\
1934 \text { Nito and Kitamura (221) } \\
1951 \text { Garner (81) }\end{array}$ \\
\hline $69-93-2$ & $\begin{array}{c}\begin{array}{c}1 H \text {-Purine-2,6,8(3H)-trione, } 7,9- \\
\text { dihydro- }\end{array} \quad\{\text { uric acid\} }\end{array}$ & $\begin{array}{l}1937 \text { Wenusch }(342) \\
1954 \text { Kosak (1) }\end{array}$ & \\
\hline $129-00-0$ & Pyrene & 1953 Cooper and Lindsey (179) & \\
\hline $110-86-1$ & Pyridine & $\begin{array}{l}1871 \text { Vohl and Eulenberg }(75,76) \\
1880 \text { LeBon }(127) \\
1894 / 95 \text { Brunton and Tunnicliffe }(482) \\
1899 \text { Thoms }(128) \\
1900 \text { Thoms }(129) \\
1903 \text { Pontag }(131) \\
1904 \text { Thoms }(277) \\
1906 \text { Warburg }(483) \\
1908 \text { Lee }(133) \\
1908 \text { Lehmann }(134) \\
1909 \text { Lehmann }(135) \\
1910 \text { Toth and Krampera (136) } \\
1914 \text { Bush }(288) \\
1929 \text { Gabelya and Kipriyanov (78) (Dr) } \\
1932 \text { McNally }(147) \\
1933 \text { Pfyl }(148) \\
1935 \text { Bastedo (187) } \\
1936 \text { Bogen (150) } \\
1936 \text { Preiss }(151,152,156) \\
1937 \text { Bradford et al. (79) } \\
1937 \text { Pyatnitskii and Kashirin (484) }\end{array}$ & $\begin{array}{l}1871 \text { Vohl and Eulenberg }(75) \\
1900 \text { Eulenburg }(486) \\
1928 \text { Shmuk (140) } \\
1929 \text { Gabelya and Kipriyanov (78) } \\
1933 \text { Dobrin (487) } \\
1936 \text { Preiss (156) } \\
1948 \text { Pyriki (166) }\end{array}$ \\
\hline
\end{tabular}




\begin{tabular}{|c|c|c|c|}
\hline CAS No. & Name (per CA Collective Index) & $\begin{array}{l}\text { Tobacco smoke and/or tobacco } \\
\text { distillate [dry (Dr), destructive (D)] }\end{array}$ & Tobacco \\
\hline $110-86-1$ & Pyridine (cont.) & $\begin{array}{l}1939 \text { Dittmar }(162,164) \\
1939 \text { Roffo }(70,71,72,73) \text { (D) } \\
1939 \text { Wenusch }(226) \\
1939 / 40 \text { de Campos }(293) \\
1942 \text { Hofmann }(485) \\
1948 \text { Pyriki }(166) \\
1951 \text { Garner }(81) \\
1954 \text { Kosak (1) }\end{array}$ & \\
\hline $27175-64-0$ & Pyridine, dimethyl- & $\begin{array}{l}1871 \text { Vohl and Eulenberg }(75) \\
1932 \text { McNally }(147) \\
1908 \text { Lehmann }(134) \\
1954 \text { Kosak (1) }\end{array}$ & $\begin{array}{l}1871 \text { Vohl and Eulenberg (75) } \\
1928 \text { Shmuk (140) }\end{array}$ \\
\hline $108-48-5$ & $\begin{array}{l}\text { Pyridine, 2,6-dimethyl- } \\
\{2,6 \text {-lutidine }\}\end{array}$ & 1932 McNally (147) & \\
\hline $1333-41-1$ & Pyridine, methyl- & $\begin{array}{l}1871 \text { Vohl and Eulenberg }(75,76) \\
1908 \text { Lehmann }(134) \\
1914 \text { Bush }(288) \\
1929 \text { Gabelya and Kipriyanov (78) (Dr) } \\
1954 \text { Kosak (1) }\end{array}$ & $\begin{array}{l}1871 \text { Vohl and Eulenberg }(75) \\
1928 \text { Shmuk (140) } \\
1929 \text { Gabelya and Kipriyanov (78) }\end{array}$ \\
\hline $109-06-8$ & Pyridine, 2-methyl- & $\begin{array}{l}1929 \text { Gabelya and Kipriyanov (78) (Dr) } \\
1932 \text { McNally (147) } \\
1944 \text { Woodward et al. (488) }\end{array}$ & 1928 Shmuk (140) \\
\hline $29611-84-5$ & Pyridine, trimethyl- & $\begin{array}{l}1871 \text { Vohl and Eulenberg }(75,76) \\
1908 \text { Lee }(133) \\
1908 \text { Lehmann }(134) \\
1954 \text { Kosak (1) }\end{array}$ & $\begin{array}{l}1871 \text { Vohl and Eulenberg (75) } \\
1928 \text { Shmuk (140) }\end{array}$ \\
\hline $108-75-8$ & $\begin{array}{l}\text { Pyridine, 2,4,6-trimethyl- } \\
\qquad\{2,4,6 \text {-collidine }\}\end{array}$ & 1932 McNally (147) & \\
\hline $532-12-7$ & $\begin{array}{l}\text { Pyridine, 3-(3,4-dihydro-2H-pyrrol- } \\
5 \text {-yl)- }\end{array}$ & $\begin{array}{l}1933 \text { Wenusch and Schöller (53) } \\
1935 \text { Wenusch and Schöller (55) } \\
1936 \text { Wenusch and Schöller (177) } \\
1939 \text { Wenusch (226) } \\
1954 \text { Kosak (1) }\end{array}$ & $\begin{array}{l}1934 \text { Wenusch and Schöller (54) } \\
1936 \text { Späth et al. (465) } \\
1944 \text { Woodward et al. (489) } \\
1946 \text { Frankenburg (250) } \\
1952 \text { Frankenburg and Gottscho (97) } \\
1953 \text { Tso and Jeffrey (117) }\end{array}$ \\
\hline & $\begin{array}{l}\text { Pyridine, 3-(3,4-dihydro-1-methyl- } \\
2 \mathrm{H} \text {-pyrrol-5-yl)- } \\
\qquad \mathrm{N} \text {-methylmyosmine }\}\end{array}$ & & $\begin{array}{l}1946 \text { Frankenburg (250) } \\
1949 \text { Swain et al. (490) } \\
1952 \text { Willaman (457) }\end{array}$ \\
\hline $24380-92-5$ & $\begin{array}{l}\text { Pyridine, 3-(1-methyl-2-piperi- } \\
\text { dinyl)-, (S)- }\{N \text {-methylanabasine }\}\end{array}$ & & $\begin{array}{l}1937 \text { Späth and Kesztler (254) } \\
1946 \text { Frankenburg }(250) \\
1953 \text { Sadykov et al. (491) }\end{array}$ \\
\hline $54-11-5$ & $\begin{array}{l}\text { Pyridine, 3-(1-methyl-2- } \\
\text { pyrrolidinyl)-, (S)- } \quad\{\text { I-nicotine }\}\end{array}$ & $\begin{array}{l}1826 \text { Unterdorden }(365)(\mathrm{Dr}) \\
1843 \text { Melsens }(492) \\
1844 \text { Melsens }(493) \\
1871 \text { Vohl and Eulenberg }(75,76) \\
1872 \text { Heubel }(494) \\
1882 \text { Kissling }(495,496) \\
1892 \text { Lowenthal }(497) \\
1898 \text { Kissling }(498) \\
1899 \text { Thoms }(128) \\
1900 \text { Thoms }(129) \\
1901 \text { Habermann }(285) \\
1902 \text { Ludwig }(499) \\
1903 \text { Pontag }(131) \\
1904 \text { Bamberger }(500) \\
1904 \text { Paschkis (501) } \\
1904 \text { Schmidt }(502) \\
1905 \text { Theodorvits }(503) \\
1906 \text { Rattner }(504) \\
1906 \text { Warburg (483) } \\
1907 \text { Bitter }(505) \\
1907 \text { Kuhles }(506) \\
1908 \text { Lee }(133) \\
1908 \text { Noda }(507) \\
1908 \text { Biederbeck (132) } \\
1908 \text { Toth (508) } \\
1909 \text { Habermann and Ehrenfeld (423) } \\
1909 \text { Toth (509) } \\
1909 \text { Weger }(510) \\
1910 \text { Toth and Krampera (136) }\end{array}$ & 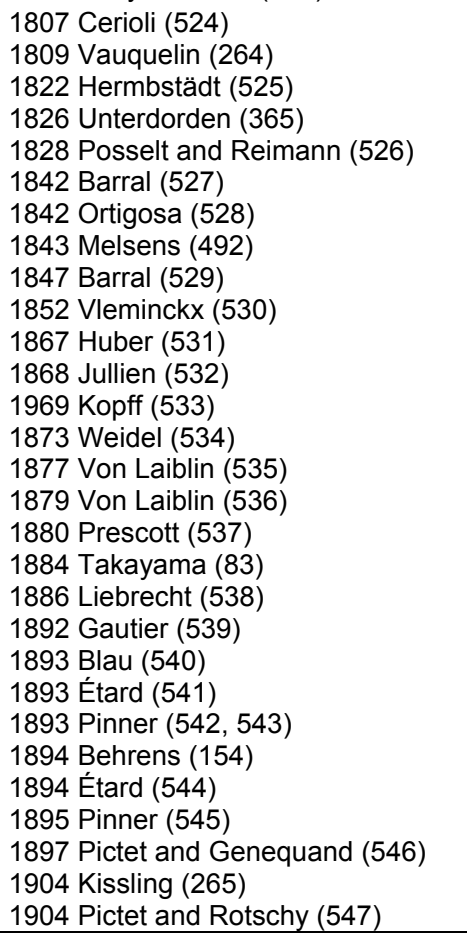 \\
\hline
\end{tabular}


Table 1. (cont.)

\begin{tabular}{|c|c|c|c|}
\hline CAS No. & Name (per CA Collective Index) & $\begin{array}{l}\text { Tobacco smoke and/or tobacco } \\
\text { distillate [dry (Dr), destructive (D)] }\end{array}$ & Tobacco \\
\hline $54-11-5$ & $\begin{array}{l}\text { Pyridine, 3-(1-methyl-2- } \\
\text { pyrrolidinyl)-, (S)- } \\
\text { (cont.) }\end{array}$ & 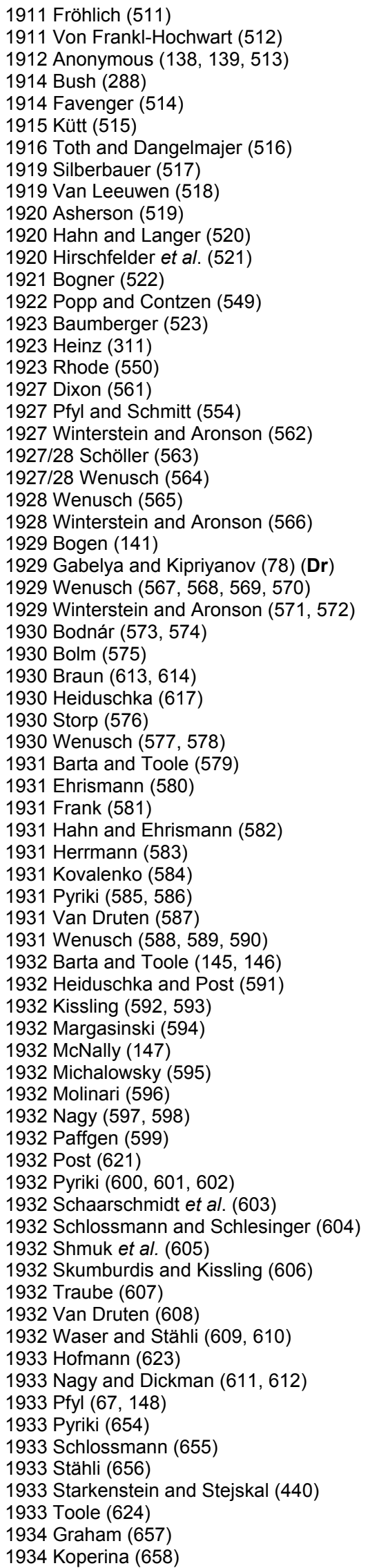 & 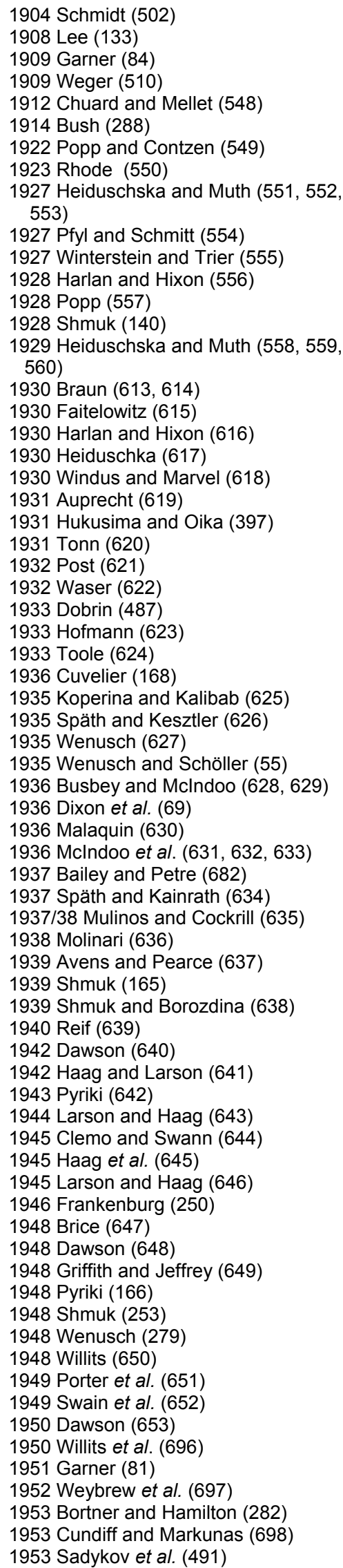 \\
\hline
\end{tabular}


Table 1. (cont.)

\begin{tabular}{|c|c|c|c|}
\hline CAS No. & Name (per CA Collective Index) & $\begin{array}{l}\text { Tobacco smoke and/or tobacco } \\
\text { distillate [dry (Dr), destructive (D)] }\end{array}$ & Tobacco \\
\hline $54-11-5$ & $\begin{array}{l}\text { Pyridine, 3-(1-methyl-2- } \\
\text { pyrrolidinyl)-, (S)- } \quad\{\text {-nicotine }\} \\
\text { (cont.) }\end{array}$ & 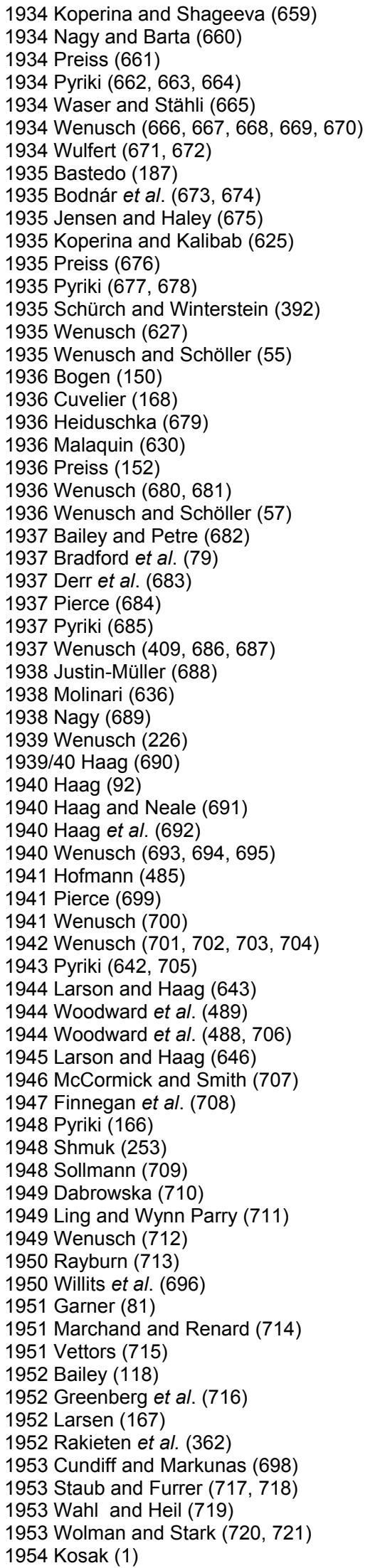 & $\begin{array}{l}1953 \text { Tso and Jeffrey (117) } \\
1953 \text { Wright and Burton (270) }\end{array}$ \\
\hline
\end{tabular}


Table 1. (cont.)

\begin{tabular}{|c|c|c|c|}
\hline CAS No. & Name (per CA Collective Index) & $\begin{array}{l}\text { Tobacco smoke and/or tobacco } \\
\text { distillate [dry (Dr), destructive (D)] }\end{array}$ & Tobacco \\
\hline \multirow[t]{2}{*}{$2820-55-5$} & $\begin{array}{l}\text { Pyridine, 3-(1-methyl-2- } \\
\text { pyrrolidinyl)-, 1-oxide, (S)- } \\
\text { \{nicotine } \mathrm{N} \text {-oxide\} }\end{array}$ & 1950 Rayburn et al. (722) & $\begin{array}{l}1885 \text { Pinner (723) } \\
1950 \text { Rayburn et al. (722) } \\
1952 \text { Frankenburg and Gottscho (97) } \\
1953 \text { Tso and Jeffrey (117) }\end{array}$ \\
\hline & $\begin{array}{l}\text { Pyridine, 3-(1-methyl-2- } \\
\text { pyrrolidinyl)-, organic acid salts }\end{array}$ & 1937 Wenusch $(724)$ & $\begin{array}{l}1809 \text { Vauquelin }(264) \\
1822 \text { Hermbstädt }(525) \\
1823 \text { Hermbstädt }(725) \\
1939 \text { Shmuk }(165)\end{array}$ \\
\hline $487-19-4$ & $\begin{array}{l}\text { Pyridine, 3-(1-methyl-1H- } \\
\text { pyrrol-2-yl)- } \quad \text { nnicotyrine\} }\end{array}$ & $\begin{array}{l}1935 \text { Wenusch and Schöller (55) } \\
1939 \text { Wenusch }(226) \\
1954 \text { Kosak (1) }\end{array}$ & $\begin{array}{l}1898 \text { Pictet and Crépieux (726) } \\
1928 \text { Wibaut and Overhoff (727) } \\
1932 \text { Wibaut and Hackman (728) } \\
1935 \text { Wenusch }(729) \\
1937 \text { Späth and Kesztler (254) } \\
1946 \text { Frankenburg }(250)\end{array}$ \\
\hline \multirow[t]{2}{*}{$494-52-0$} & $\begin{array}{l}\text { Pyridine, 3-(2-piperidinyl)-, (S)- } \\
\{\text { l-anabasine }\}\end{array}$ & 1933 Haag (730) & $\begin{array}{l}1931 \text { Ehrenstein (111) } \\
1931 \text { Smith (731) } \\
1932 \text { Smith (732) } \\
1935 \text { Olchansky (733) } \\
1935 \text { Smith (734) } \\
1937 \text { Späth and Kesztler (251) } \\
1939 \text { Shmuk and Borozdina (638) } \\
1942 \text { Smith and Smith (735) } \\
1946 \text { Dubinin and Chelintsev (736) } \\
1946 \text { Frankenburg (250) } \\
1948 \text { Matveev (737) } \\
1048 \text { Sadykov et al. (738) } \\
1948 \text { Shmuk (253) } \\
1949 \text { Porter et al. (651) } \\
1951 \text { Garner (81) } \\
1953 \text { Sadykov et al. (491) } \\
1953 \text { Tso and Jeffrey (117) }\end{array}$ \\
\hline & $\begin{array}{r}\text { Pyridine, 3-(2-piperidinyl)-, 6-oxo- } \\
\{6 \text {-oxoanabasine }\}\end{array}$ & & 1939 Henry (176) \\
\hline $494-97-3$ & $\begin{array}{l}\text { Pyridine, 3-(2-pyrrolidinyl)-, (S)- } \\
\{\text { I-nornicotine }\}\end{array}$ & $\begin{array}{l}1944 \text { Larson and Haag (643) } \\
1945 \text { Larson and Haag (646) }\end{array}$ & $\begin{array}{l}1928 \text { Shmuk (140) } \\
1935 \text { Späth and Zajic (739) } \\
1936 \text { Wenusch (740) } \\
1936 \text { Späth et al. (741) } \\
1937 \text { Späth and Kesztler (251) } \\
1944 \text { Larson and Haag (643) } \\
1945 \text { Larson and Haag (646) } \\
1946 \text { Frankenburg (250) } \\
1948 \text { Brice (647) } \\
1948 \text { Dawson (648) } \\
1948 \text { Shmuk (253) } \\
1949 \text { Swain et al. (652) } \\
1951 \text { Garner (81) } \\
1952 \text { Weybrew et al. (697) } \\
1953 \text { Tso and Jeffrey (117) } \\
1953 \text { Wright and Burton (270) }\end{array}$ \\
\hline \multirow[t]{2}{*}{$494-98-4$} & $\begin{array}{r}\text { Pyridine, 3-(1H-pyrrol-2-yl)- } \\
\text { \{nornicotyrine\} }\end{array}$ & & 1946 Frankenburg (250) \\
\hline & Pyridine bases & $\begin{array}{l}1932 \text { McNally (147) } \\
1954 \text { Kosak (1) }\end{array}$ & \\
\hline $98-92-0$ & $\begin{array}{l}\text { 3-Pyridinecarboxamide } \\
\qquad \text { \{nicotinamide\} }\end{array}$ & & 1953 Tso and Jeffrey (117) \\
\hline $100-54-9$ & $\begin{array}{l}\text { 3-Pyridinecarbonitrile } \\
\qquad\{\text { nicotinonitrile\} }\end{array}$ & 1944 Woodward et al. (488) & \\
\hline $59-67-6$ & $\begin{array}{l}\text { 3-Pyridinecarboxylic acid } \\
\qquad\{\text { nicotinic acid\} }\end{array}$ & 1944 Woodward et al. (488) & $\begin{array}{l}1879 \text { Von Laiblin (536) } \\
1938 \text { Chen et al. (742) } \\
1952 \text { Gottscho and Frankenburg (743) } \\
1953 \text { Tso and Jeffrey (117) }\end{array}$ \\
\hline 289-95-2 & $\{1,3$-diazine $\}$ & & 1950 Steinberg $(260)$ \\
\hline $107-49-3$ & $\begin{array}{l}\text { Pyrophosphoric acid, tetraethyl } \\
\text { ester }\{\text { TEPP\} } \\
\text { a metabolite of phosphorus- } \\
\text { containing pesticides }\end{array}$ & & 1951 Vinzant (60) \\
\hline $109-97-7$ & Pyrrole & $\begin{array}{l}1909 \text { Lehmann (135) } \\
1929 \text { Gabelya and Kipriyanov (78) (Dr) } \\
1939 \text { Roffo }(70,71,72,73)(\mathrm{D}) \\
1939 / 40 \text { de Campos }(293) \\
1954 \text { Kosak (1) }\end{array}$ & $\begin{array}{l}1928 \text { Shmuk (140) } \\
1937 \text { Fromm (157) }\end{array}$ \\
\hline
\end{tabular}


Table 1. (cont.)

\begin{tabular}{|c|c|c|c|}
\hline CAS No. & Name (per CA Collective Index) & $\begin{array}{l}\text { Tobacco smoke and/or tobacco } \\
\text { distillate [dry (Dr), destructive (D)] }\end{array}$ & Tobacco \\
\hline & Pyrroles, structure unspecified & $\begin{array}{l}1932 \text { McNally }(147) \\
1937 \text { Fromm }(157) \\
1954 \text { Kosak (1) }\end{array}$ & \\
\hline $123-75-1$ & Pyrrolidine & 1909 Lehmann (135) & $\begin{array}{l}1908 \text { Pictet and Court (110) } \\
1928 \text { Shmuk (140) }\end{array}$ \\
\hline $120-94-5$ & Pyrrolidine, $N$-methyl- & $\begin{array}{l}1932 \text { McNally (147) } \\
1954 \text { Kosak (1) }\end{array}$ & $\begin{array}{l}1905 \text { Pictet (744) } \\
1908 \text { Pictet and Court (110) } \\
1928 \text { Shmuk (140) } \\
1939 \text { Späth and Biniecki (249) }\end{array}$ \\
\hline $91-22-5$ & Quinoline & $\begin{array}{l}1929 \text { Gabelya and Kipriyanov (78) (Dr) } \\
1944 \text { Woodward et al. (488) }\end{array}$ & \\
\hline $7440-14-4$ & Radium & & 1937 Drobkov (103) \\
\hline \multirow[t]{2}{*}{$10043-92-2$} & Radon & & 1937 Drobkov (103) \\
\hline & Reconstituted tobacco sheet & & 1857 Von der Porten (745) \\
\hline \multirow[t]{4}{*}{$9037-80-3$} & Reductase & & $\begin{array}{l}1913 \text { Oosthuizen and Shedd (170) } \\
1946 \text { Garner }(173) \\
1951 \text { Garner }(81)\end{array}$ \\
\hline & Resin & $\begin{array}{l}1893 \text { Kissling }(223) \\
1932 \text { Koperina }(746) \\
1934 \text { Koperina }(747) \\
1935 \text { Wenusch }(278,748) \\
1937 \text { Wenusch }(342,749) \\
1949 \text { Dabrowska }(710) \\
1950 \text { Mazzulli }(750) \\
1954 \text { Kosak }(1)\end{array}$ & $\begin{array}{l}1904 \text { Kissling }(265) \\
1914 \text { von Degrazia (751) } \\
1924 \text { Shmuk }(85) \\
1931 \text { Hukusima and Oika (397) } \\
1935 \text { Shirokaya }(412) \\
1938 \text { Gaertner }(752) \\
1940 \text { Pyriki }(753)\end{array}$ \\
\hline & Resin acids & $\begin{array}{l}1893 \text { Kissling (223) } \\
1954 \text { Kosak (1) }\end{array}$ & \\
\hline & Resinol & & 1924 Shmuk (85) \\
\hline \multirow[t]{2}{*}{$3615-41-6$} & Rhamnose & & 1936 Neuberg and Kobel (233) \\
\hline & Rhoeadin & & 1936 Späth and Zajic (169) \\
\hline $83-88-5$ & Riboflavin & & 1952 Jensen (161) \\
\hline \multirow[t]{2}{*}{$7440-17-7$} & Rubidium & & $\begin{array}{l}1862 / 63 \text { Grandeau }(335) \\
1950 \text { Yamagata }(334)\end{array}$ \\
\hline & Saccharides & 1940 Dittmar (754) & $\begin{array}{l}1940 \text { Dittmar (754) } \\
1941 \text { Pyriki }(453)\end{array}$ \\
\hline 129990-04-1 & Saponin (from tobacco) & & 1935 Kobel and Neuberg (235) \\
\hline $7782-49-2$ & Selenium & & 1933 Martin and Trelease (755) \\
\hline $6898-95-9$ & Serine & & $\begin{array}{l}1952 \text { Frankenburg and Gottscho (97) } \\
1953 \text { Pearse and Novellie (98) } \\
1953 \text { Zacharius and Frankenburg (99) }\end{array}$ \\
\hline $7631-86-9$ & Silica & & 1894 Behrens (154) \\
\hline $7440-21-3$ & Silicon & 1952 Bailey (118) & $\begin{array}{l}1925 \text { Maclntyre et al. (756) } \\
1928 \text { Bailey and Anderson (281) } \\
1951 \text { Garner (81) }\end{array}$ \\
\hline \multirow[t]{4}{*}{$7440-22-4$} & Silver & & 1936 Johnson (350) \\
\hline & $\begin{array}{c}\alpha-\text { Socratine }=\{\text { nicotyrine }+2,3 \\
\text { bipyridine }+ \text { - nicotinic acid }+ \\
\text { nornicotine }+ \text { anatabine }\}(58)\end{array}$ & $\begin{array}{l}1919 \text { Kissling (477) } \\
1933 \text { Wenusch and Schöller (53) } \\
1935 \text { Wenusch and Schöller (55) } \\
1936 \text { Wenusch and Schöller (177) } \\
1954 \text { Kosak (1) }\end{array}$ & $\begin{array}{l}1934 \text { Wenusch and Schöller (54) } \\
1935 \text { Wenusch and Schöller (55) }\end{array}$ \\
\hline & $\begin{array}{l}\beta \text {-Socratine }=\{\text { nicotyrine }+2,3 \text { - } \\
\text { bipyridine }+ \text { nicotinic acid }+ \\
\text { nornicotine }+ \text { anatabine }\}(58)\end{array}$ & $\begin{array}{l}1919 \text { Kissling (477) } \\
1933 \text { Wenusch and Schöller (53) } \\
1935 \text { Wenusch and Schöller (55) } \\
1936 \text { Wenusch and Schöller (177) } \\
1954 \text { Kosak (1) }\end{array}$ & $\begin{array}{l}1934 \text { Wenusch and Schöller (54) } \\
1935 \text { Wenusch and Schöller (55) }\end{array}$ \\
\hline & $\gamma$-Socratine $=1$-nornicotine $(58)$ & $\begin{array}{l}1919 \text { Kissling (477) } \\
1933 \text { Wenusch and Schöller (53) } \\
1935 \text { Wenusch and Schöller (55) } \\
1936 \text { Wenusch and Schöller (177) } \\
1954 \text { Kosak (1) }\end{array}$ & $\begin{array}{l}1928 \text { Shmuk (140) } \\
1934 \text { Wenusch and Schöller (54) } \\
1935 \text { Wenusch and Schöller (55) }\end{array}$ \\
\hline $7440-23-5$ & Sodium & 1952 Bailey (118) & $\begin{array}{l}1928 \text { Bailey and Anderson (281) } \\
1938 \text { Gaertner }(752) \\
1950 \text { Frankenburg }(757) \\
1950 \text { Yamagata }(334) \\
1951 \text { Garner }(81) \\
1953 \text { Wolff et al. }(208)\end{array}$ \\
\hline
\end{tabular}


Table 1. (cont.)

\begin{tabular}{|c|c|c|c|}
\hline CAS No. & Name (per CA Collective Index) & $\begin{array}{l}\text { Tobacco smoke and/or tobacco } \\
\text { distillate [dry (Dr), destructive (D)] }\end{array}$ & Tobacco \\
\hline $9005-25-8$ & Starch & & $\begin{array}{l}1885 \text { Müller-Thurgen (153) } \\
1894 \text { Behrens (154) } \\
1914 \text { Garner et al. (100) } \\
1934 \text { Pyriki (331) } \\
1935 \text { Koenig (89) } \\
1942 \text { Ward (172) } \\
1951 \text { Bacon et al. (267) } \\
1951 \text { Garner (81) } \\
1952 \text { Bacon et al. (268) } \\
1952 \text { Willaman (457) } \\
1953 \text { Phillips and Bacot (269) }\end{array}$ \\
\hline $7440-24-6$ & Strontium & 1952 Bailey (118) & $\begin{array}{l}1897 \text { Trimble }(758) \\
1921 \text { Headden }(220)\end{array}$ \\
\hline $57-50-1$ & Sugar & & $\begin{array}{l}1885 \text { Müller-Thurgen (153) } \\
1914 \text { Garner et al. (100) } \\
1934 \text { Pyriki (331) } \\
1935 \text { Koenig (89) } \\
1936 \text { Dixon et al. (69) } \\
1951 \text { Garner (81) }\end{array}$ \\
\hline $8013-17-0$ & Sugar, invert & & 1951 Garner (81) \\
\hline $14808-79-8$ & Sulfate & & $\begin{array}{l}1923 \text { Garner et al. (325) } \\
1935 \text { Koenig (89) } \\
1951 \text { Garner (81) }\end{array}$ \\
\hline $7704-34-9$ & Sulfur & & $\begin{array}{l}1914 \text { Shedd (759) } \\
1928 \text { Bailey and Anderson (281) } \\
1934 \text { Heiserich (404) } \\
1937 \text { McMurtrey (441) } \\
1941 \text { Ward (339) } \\
1951 \text { Garner (81) }\end{array}$ \\
\hline $71010-48-5$ & $\alpha$-Tabacenic acid $\{\alpha$-tobacco acid $\}$ & & $\begin{array}{l}1914 \text { von Degrazia }(751) \\
1924 \text { Shmuk }(85) \\
1934 \text { Shmuk and Shirokaya (455) } \\
1951 \text { Garner (81) }\end{array}$ \\
\hline $71010-46-3$ & $\beta$-Tabacenic acid $\quad\{\beta$-tobacco acid $\}$ & & $\begin{array}{l}1914 \text { von Degrazia }(751) \\
1924 \text { Shmuk }(85) \\
1934 \text { Shmuk and Shirokaya (455) } \\
1951 \text { Garner }(81)\end{array}$ \\
\hline 71010-47-4 & $\gamma$-Tabacenic acid $\quad\{\gamma$-tobacco acid $\}$ & & $\begin{array}{l}1914 \text { von Degrazia }(751) \\
1924 \text { Shmuk }(85) \\
1934 \text { Shmuk and Shirokaya (455) } \\
1951 \text { Garner }(81)\end{array}$ \\
\hline $1401-55-4$ & $\{\operatorname{tannic}$ acid\} & & $\begin{array}{l}1894 \text { Behrens (154) } \\
1924 \text { Shmuk (85) } \\
1935 \text { Koenig (89) } \\
1953 \text { Phillips and Bacot (269) }\end{array}$ \\
\hline 494-04-2 & $3,2^{\prime}: 4^{\prime}, 3^{\prime \prime}-$ Terpyridine $\quad$ \{nicotelline & & $\begin{array}{l}1901 \text { Pictet and Rotschy (108) } \\
1914 \text { Noga }(443) \\
1928 \text { Shmuk (140) }\end{array}$ \\
\hline $544-63-8$ & $\begin{array}{r}\text { Tetradecanoic acid } \\
\qquad \text { \{myristic acid\} }\end{array}$ & & 1944 Venkatarao et al. (466) \\
\hline $7440-28-0$ & Thallium & & $\begin{array}{l}1932 \text { McMurtrey }(760) \\
1934 \text { Heffer et al. }(180) \\
1938 \text { Van Der Veen }(761) \\
1940 \text { Shear and Ussery (762) }\end{array}$ \\
\hline $59-43-8$ & $\begin{array}{l}\text { Thiazolium, 3-[(4-amino-2-methyl- } \\
\text { 5-pyrimidinyl)methyl]-5-(2- } \\
\text { hydroxyethyl)-4-methyl-, chloride } \\
\text { \{thiamine\} }\end{array}$ & & 1952 Jensen (161) \\
\hline $463-56-9$ & Thiocyanic acid & $\begin{array}{l}1938 \text { Schöller (426) } \\
1954 \text { Kosak (1) }\end{array}$ & $\begin{array}{l}1938 \text { Schöller (426) } \\
1950 \text { Molinari (159) }\end{array}$ \\
\hline $505-14-6$ & Thiocyanogen & 1909 Toth $(763)$ & \\
\hline $72-19-5$ & Threonine & & $\begin{array}{l}1953 \text { Pearse and Novellie (98) } \\
1953 \text { Zacharius and Frankenburg (99) }\end{array}$ \\
\hline $7440-31-5$ & Tin & & $\begin{array}{l}1934 \text { Heffer et al. (180) } \\
1941 \text { Ward (339) }\end{array}$ \\
\hline $7440-32-6$ & Titanium & 1952 Bailey (118) & $\begin{array}{l}1913 \text { Traetta-Mosca }(217) \\
1921 \text { Headden }(220) \\
1941 \text { Ward (339) }\end{array}$ \\
\hline & Tobacco, combustion products & & 1898 Cavalli (764) \\
\hline
\end{tabular}


Table 1. (cont.)

\begin{tabular}{|c|c|c|c|}
\hline CAS No. & Name (per CA Collective Index) & $\begin{array}{l}\text { Tobacco smoke and/or tobacco } \\
\text { distillate [dry (Dr), destructive (D)] }\end{array}$ & Tobacco \\
\hline & Tobacco, general & & $\begin{array}{l}1793 \text { Murray }(765) \\
1895 \text { Carpenter }(766) \\
1905 \text { Kissling }(767) \\
1907 \text { Lehmann }(768) \\
1908 \text { Kissling }(769) \\
1910 \text { Kissling }(770) \\
1913 \text { Traetta-Mosca (474) } \\
1919 \text { Kissling (477) } \\
1930 \text { Ehrenstein }(771) \\
1930 \text { Gabelya and Kipriyanov (772) } \\
1931 \text { Shmuk (773) } \\
1934 \text { Garner et al. (283) } \\
1935 \text { Waser }(774) \\
1935 \text { Wenusch (775) } \\
1936 \text { Brückner (332) } \\
1937 \text { Schöller and Molinari (776) } \\
1938 \text { Brandt }(777,778) \\
1939 \text { Gaertner (158) } \\
1940 \text { Bodnár and Votisky (779) } \\
1940 \text { Smirnov et al. (780) } \\
1941 \text { Palfray et al. (781) } \\
1943 \text { Pyriki (782) } \\
1950 \text { Pyriki (783) }\end{array}$ \\
\hline & Tobacco smoke, general & $\begin{array}{l}1923 \text { Baumberger }(784) \\
1930 \text { Gabelya and Kipriyanov }(772) \\
1931 \text { Koperina }(785) \\
1933 \text { Kurilo }(786) \\
1934 \text { Tschebull }(787) \\
1937 \text { Pyriki }(788) \\
1937 \text { Rohrbach }(789) \\
1937 \text { Wenusch }(724,790) \\
1938 \text { Brandt }(777,778) \\
1939 \text { Smirnov and Sirotenko }(791) \\
1939 \text { Wenusch }(792) \\
1940 \text { Bodnár and Votisky }(779) \\
1941 \text { Hillsman }(793) \\
1941 \text { Matthews }(794) \\
1946 \text { Fromm }(795) \\
1948 \text { Matthews }(796) \\
1951 \text { Laskowski }(797) \\
1953 \text { Warner and Hobbs }(798)\end{array}$ & \\
\hline $638-68-6$ & $\begin{array}{l}\text { Triacontane } \\
\text { 17-Tritriacontanone }\end{array}$ & $\begin{array}{l}1935 \text { Schürch and Winterstein (392) } \\
1954 \text { Kosak (1) }\end{array}$ & 1937 Shmuk (413) \\
\hline & Triglycerides & & 1944 Venkatarao et al. (466) \\
\hline $73-22-3$ & Tryptophan & & $\begin{array}{l}1953 \text { Pearse and Novellie (98) } \\
1953 \text { Zacharius and Frankenburg (99) }\end{array}$ \\
\hline & Tyrosinase & & 1953 Barrett et al. (175) \\
\hline $60-18-4$ & Tyrosine & & $\begin{array}{l}1952 \text { Frankenburg and Gottscho (97) } \\
1953 \text { Pearse and Novellie (98) } \\
1953 \text { Zacharius and Frankenburg (99) }\end{array}$ \\
\hline $112-37-8$ & Undecanoic acid & 1952 James and Martin (82) & \\
\hline $7440-61-1$ & Uranium & & $\begin{array}{l}1937 \text { Drobkov }(103) \\
1949 \text { Drobkov }(799)\end{array}$ \\
\hline $97-59-6$ & $\begin{array}{r}\text { Urea, (2,5-dioxo-4-imidazolidinyl)- } \\
\text { \{allantoin\} }\end{array}$ & & $\begin{array}{l}1933 \text { Vickery et al. (182) } \\
1935 \text { Vickery et al. (183) } \\
1946 \text { Garner (173) } \\
1951 \text { Garner (81) }\end{array}$ \\
\hline $9002-13-5$ & $\begin{array}{l}\text { Urease } \\
\text { Uronic acids }\end{array}$ & & $\begin{array}{l}1953 \text { Barrett et al. (175) } \\
1935 \text { Kobel and Neuberg (235) } \\
1953 \text { Phillips and Bacot }(800)\end{array}$ \\
\hline 7004-03-7 & Valine & & $\begin{array}{l}1952 \text { Frankenburg and Gottscho (97) } \\
1953 \text { Zacharius and Frankenburg (99) }\end{array}$ \\
\hline $7440-62-2$ & Vanadium & & 1941 Ward (339) \\
\hline $7732-18-5$ & Water & $\begin{array}{l}1857 \text { Vogel }(123) \\
1929 \text { Gabelya and Kipriyanov (78) (Dr) } \\
1930 \text { Braun }(613,614) \\
1932 \text { McNally }(147) \\
1940 \text { Haag }(92)\end{array}$ & $\begin{array}{l}1884 \text { Takayama }(83) \\
1936 \text { Dixon et al. }(69) \\
1948 \text { Barnhardt }(801)\end{array}$ \\
\hline
\end{tabular}


Table 1. (cont.)

\begin{tabular}{|c|c|c|c|}
\hline CAS No. & Name (per CA Collective Index) & $\begin{array}{l}\text { Tobacco smoke and/or tobacco } \\
\text { distillate [dry (Dr), destructive (D)] }\end{array}$ & Tobacco \\
\hline $7440-66-6$ & Zinc & & $\begin{array}{l}1926 \text { Sommer and Lipman (256) } \\
1934 \text { Heffer et al. (180) } \\
1938 \text { McMurtrey and Robinson (120) } \\
1941 \text { Ward (339) } \\
1951 \text { Garner (81) } \\
1952 \text { Nicholas (351) }\end{array}$ \\
\hline
\end{tabular}

While noting that both ammonia and nicotine were previously identified as tobacco smoke components, KOSAK elected not to list references to them because their number of references, in his opinion, was too numerous to list. Also the number of references to carbon dioxide and carbon monoxide were limited by KOSAK. Table 1 lists many of the pre-1954 references to ammonia, nicotine, carbon dioxide, and carbon monoxide in tobacco and/or tobacco smoke. Included in Table 1 are several pesticide residues, e.g., arsenic/arsenic oxide, Toxaphene ${ }^{\circledR}$, Lindane ${ }^{\circledR}$, Parathion ${ }^{\circledR}$, DDT, and TEPP (tetraethyl pyrophosphate), plus several pesticide residue degradation products, e.g., $o, p$ 'DDD and $m, p$ '-DDD, identified by VINZANT in 1951 (60). Listed in Table 1 are a few compounds used as flavorants on tobacco products, most notably menthol which, however, occurs naturally in trace amounts in several tobacco types. Table 1 also cites numerous references to pre-1954 studies of a general nature. These include references to arsenical insecticides, alkaloids, aliphatic acids, aliphatic hydrocarbons amino acids, bases, tobacco distillates, tobacco fats, chlorophyll degradation products, enzymes (general information), hydrocarbons, hygroscopic agents, paraffins, pigments, sugars, tobacco combustion products, tobacco (general), tobacco smoke (general), and triglycerides. These items are not included in the total of 325.

Among metals, nonmetals, and ions, KOSAK in his 1954 catalogue listed only arsenic plus four ions as smoke components and KOSAK questioned the identification of each of the latter. Examination of Table 1 reveals that over 50 metals, nonmetals, and ions were identified in tobacco prior to late 1953 . However, it was post-1953 when a concerted effort was made to determine the transfer of numerous metals from tobacco to tobacco smoke during the smoking process, e.g., the 1957 study by CogBILL and HoBBS (61). The references for numerous additional studies of this type are listed by Rodgman and PERFETTI [see Chapter XX in (2)]. Among the metals and nonmetals in tobacco smoke, the one much studied pre-1954 was of course arsenic (As). Between 1917 and 1951, the As level in tobacco rose from about 12 to $57 \mu \mathrm{g} / \mathrm{g}$ (62). As, usually considered as $\mathrm{As}_{2} \mathrm{O}_{3}$ in tobacco, was removed from tobacco agronomy in 1952.

While our REFERENCES section contains numerous references on the isolation, identification, and quantitation of many of the tobacco components listed in Table 1, also available are literally hundreds of pre-1954 references on studies that dealt with the effect of several components in tobacco smoke, e.g., nicotine, carbon monoxide, or in chewing tobacco, e.g., nicotine and related alkaloids, on a host of human biological activities. Many such studies are catalogued in the 1961 LARSON et al. book on experimental and clinical studies on tobacco (63).
In Table 1 are listed two dozen enzymes identified in tobacco prior to 1954 . This early research was done primarily to obtain an insight into the mechanism of tobacco curing, fermentation, and aging. With the subsequent escalation of technologies pertinent to the isolation and characterization of enzymes, their number in plants, including the various types of tobacco, has increased dramatically. The number of completely identified tobacco enzymes listed by RODGMAN and PERFETTI approximated 500 but many thousands of enzymes present in the tobaccos have been catalogued [see Chapter XXII in (2)].

Paralleling the increase in identified chemical components in tobacco smoke from the fewer than 100 in 1954 (1) to more than 5200 recently catalogued (2) has been the increase in identified chemical components of tobacco from 325 (see Table 1) to the nearly 5000 recently catalogued (2).

\section{CONTROVERSIES OVER THE ROFFO INVESTIGATIONS}

Because the results of a 1930 biological study reported by ROFFO (802) on a tobacco-related material, a destructive distillate from tobacco, coincided with the demonstration of the tumorigenicity to rodent skin of the $\mathrm{PAH} \mathrm{DB}[a, h] \mathrm{A}$ in 1930 (803) and preceded the demonstration of similar tumorigenicity of $\mathrm{B}[a] \mathrm{P}$ in 1932 (804), questions were frequently raised about the pertinence to tobacco smoke of the subsequent ROFFO findings in which the destructive distillate tar was not only reported to be tumorigenic to laboratory animal skin but also to contain the tumorigenic $\mathrm{B}[a] \mathrm{P}$ (see Table 2).

In their comments on the RoFFOs' reports of their investigations on the chemical composition and biological properties of a destructive distillate from tobacco and the presence of $\mathrm{B}[a] \mathrm{P}$ in the distillate (claims based on spectroscopic data), WYNDER et al. (5) noted that ROFFO had claimed to have identified $\mathrm{B}[a] \mathrm{P}$ in the tobacco destructive distillate, but his finding was not confirmed by E. HOFFMANN et al. (10). However, WYNDER et al. did note that EBY (805), in his examination of the cigarette tar used in the WYNDER et al. 1953 biological study, did not detect $\mathrm{B}[a] \mathrm{P}$. They made little comment about EBY's failure to identify $\mathrm{B}[a] \mathrm{P}$ or any other tumorigenic $\mathrm{PAH}$ in the WYNDER et al. cigarette tar or the 1957 summary by FIESER (806) on 1) the inadequacy of the spectroscopic data purported to indicate the presence of $\mathrm{B}[a] \mathrm{P}$ in cigarette tar or 2) the failure of FIESER's colleagues to detect $\mathrm{B}[a] \mathrm{P}$ in cigarette tar despite their success in identifying $\mathrm{B}[a] \mathrm{P}$ in roast coffee. 
Table 2. Comparison of RofFo's findings on the destructive distillate of tobacco vs. research findings reported by others on tobacco smoke

\begin{tabular}{|c|c|}
\hline $\begin{array}{l}\text { Roffo and the destructive distillate of tobacco, other related } \\
\text { research }\end{array}$ & Tobacco smoke and other related research \\
\hline $\begin{array}{l}\text { Roffo }(320,811) \text { defined CO derived from tobacco as a } \\
\text { hazard. }\end{array}$ & $\begin{array}{l}\text { Many investigators pre-1954 (see Table 1) and post-1953 proposed } \\
\text { the CO in tobacco smoke as a hazard. Subsequently, because of its } \\
\text { hazard, CO became categorized as a "Hoffmann analyte" [see } \\
\text { Chapter XXIII in (2)]. }\end{array}$ \\
\hline A.E. Roffo $(245,812)$ identified several PAHs (anthracene, & In 1953, several PAHs (anthracene, pyrene) were identified in \\
\hline
\end{tabular}

$\mathrm{B}[\mathrm{a}] \mathrm{P}$, phenanthrene) in the destructive distillate of tobacco.

$\mathrm{B}[\mathrm{a}] \mathrm{P}$ was identified in the destructive distillate of tobacco

$(178,240,246,247,248,812)$.

Note: The Roffos reported 1,2-benzpyrene in the tobacco

destructive distillate. 1,2-Benzpyrene was the name originally assigned by Cook et al. (804) to their coal-tar isolate. Subsequently, the nomenclature 1,2-benzpyrene was changed to 3,4-benzpyrene and then to benzo[a]pyrene by the International Union of Pure and Applied Chemistry (IUPAC). cigarette smoke by R.I. Cooper and Lindsey (179).

- E.A. Cooper et al. (10) stated that they could not confirm Roffo's finding of B[a]P in the destructive distillate of tobacco.

- However, it should also be noted that Eby (805) could not detect $B[a] P$ in the "tar" used by Wynder et al. (5) in their noted 1953 biological study.

- In 1957, Fieser (806) classified as inadequate the ultraviolet spectrographic data claimed by R.L. Cooper and Lindsey (814) and by Rand personnel $(815,816,817)$ as indicating $\mathrm{B}[\mathrm{a}] \mathrm{P}$ in cigarette "tar".

- Fieser's colleague could not detect B[a]P in cigarette "tar" but identified it in roast coffee (806).

- $\mathrm{B}[a] \mathrm{P}$ was eventually accepted universally as a cigarette smoke component after the published report in 1959 of its isolation in crystalline form from cigarette smoke by Wynder and Hoffmann (818).

- Fourteen PAHs, including benz[a]anthracene (B[a]A), B[a]P, and $\mathrm{DB}[a, h] \mathrm{A}$ had previously been isolated individually from cigarette smoke (819), 12 in crystalline form, two as oils [see Table 1 in Rodgman (820)].

In 1930 and 1939 , Roffo reported the tumorigenicity to animal skin of the destructive distillate of tobacco $(70,72,802,821$, $822,823,824)$.

Roffo reported that the specific tumorigenicities of the destructive distillates from the different tobacco types were essentially the same $(70,71,72,73,825)$.

Roffo (827) claimed that the destructive distillate "tar" from tobacco showed greater specific tumorigenicity to laboratory animals than did coal tar.

The yields of $\mathrm{B}[\mathrm{a}] \mathrm{P}$ and other PAHs in the destructive distillate from organic solvent-extracted tobacco were less than those in the destructive distillate from the control (unextracted) tobacco (248).

The destructive distillate from the organic solvent-extracted tobacco showed less tumorigenicity than the destructive distillate from the control (unextracted) tobacco (248).

Roffo proposed that the precursor in tobacco of the PAHs in the destructive distillate from tobacco (248) was phytosterol. Note: In 1939 and 1941, cholesterol was not a known component of tobacco. Also, high molecular weight terpenoids such as solanesol, neophytadiene, and the cyclotetradecane derivatives had not yet been identified as tobacco components. Each cyclotetradecane derivative had a 14-carbon structure similar to that in anthracene or phenanthrene. Subsequently, the high molecular weight terpenoids and derivatives (solanesyl esters) were identified in tobacco (and tobacco smoke) as were cyclotetradecane derivatives plus numerous phytosterols and their longchained aliphatic esters plus cholesterol, its glycoside, and many long-chained aliphatic cholesteryl esters.

In 1953, cigarette "tar" was reported to be tumorigenic to mouse skin in the renowned study by Wynder et al. (3-5) and subsequently reported to be tumorigenic to several other species [see Tables VII $1-3$ in (808)].

As reported by Wynder and Hoffmann in 1963 (826), subsequent findings with tobacco smoke did differ from those reported by Roffo $(70,71,72,73,825)$. The specific tumorigenicities (mouse skin) of the cigarette "tars" from different tobacco types varied as follows: flue-cured = Oriental $>$ Maryland $>$ burley.

The $\mathrm{B}[\mathrm{a}] \mathrm{P}$ levels in the CSC varied as follows: flue-cured $>$ Oriental $>$ Maryland $>$ burley

Later, Jaffe et al. (828) and Hueper (829) described findings that disagreed with those of Roffo, i.e., they reported that coal tar showed greater specific tumorigenicity to laboratory animals than did tobacco "tar".

The per cigarette yields of $\mathrm{B}[\mathrm{a}] \mathrm{P}$ and other $\mathrm{PAHs}$ were less in the "tar" [cigarette smoke condensate (CSC)] from organic solventextracted tobacco than those in the CSC from control (unextracted) tobacco $(830,831,832,833)$ [see Table 3 in Rodgman (820)].

The CSC from organic solvent-extracted tobacco showed lower specific tumorigenicity than CSC from control tobacco but the $\%$ decrease in specific tumorigenicity was less than the \% decrease in the B[a]P content (833) of the CSC.

- Subsequently, identified in tobacco were several phytosterols [stigmasterol (834), sitosterol (835), campesterol (836), ergosterol $(835)]$ and cholesterol $(837,838)$ and their longchained aliphatic esters (839). All were also identified in tobacco smoke components $(838,840,841)$.

- The saturated aliphatic hydrocarbons ${ }^{a}$, the phytosterols ${ }^{b}$, and terpenoids ${ }^{c}$ (such as solanesol and its long-chained aliphatic esters) in tobacco, were demonstrated to be major precursors of PAHs in CSC in a 1979 study by Severson et al. (842) of the United States Department of Agriculture and in a 1958 study in which the levels of each category in the cigarette tobacco were doubled and tripled (843).

- In 1963 and 1964, Rowland et al. (844) described the conversion of a cyclotetradecane derivative to a phenanthrene derivative. 
Table 2. (cont.) Roffo and the destructive distillate of tobacco, other related
research

In 1939, Roffo demonstrated that the pyrolysate from cholesterol was tumorigenic $(345,347)$, a confirmation of the 1928 findings of Kennaway and Sampson (343) and a prelude to the 1949 findings of Falk et al. (845).

Note: In 1939 and 1941, cholesterol was not a known component of tobacco.

Roffo reported that the tumorigenicity of his cholesterol pyrolysate was due to derivatives of phenanthrene (345).
Tobacco smoke and other related research

A cholesterol pyrolysate was demonstrated to be tumorigenic in 1928 by Kennaway and Sampson (343), in 1945 by Beck et al. (846), and again in 1949 by Falk et al. (845).

- Pyrolysis of cholesterol and cholesteryl esters yielded two tumorigenic compounds, 4-cholesten-3-one and 3,5cholestadiene $(845,847)$, both of which may be considered as phenanthrene derivatives.

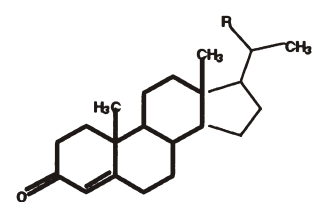

4-Cholesten-3-one

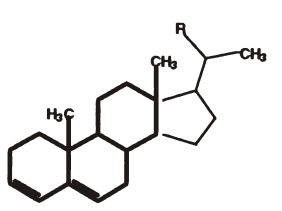

3,5-Cholestadiene
- In the 1935 report on his pyrolysis of cholesterol, Cohen et al. (848) described the generation of phenanthrene derivatives, the so-called Diels' hydrocarbon (17-methyl$17 \mathrm{H}$-cyclopenta[a]phenanthrene) and chrysene. At one time, chrysene was classified as a carcinogenic by the International Agency for Research on Cancer (IARC) (849) but subsequently the IARC cancelled its carcinogen classification.

- By 1951, many phenanthrene derivatives had been reported as tumorigenic, e.g., 1,2,3,4-tetramethylphenanthrene (850).

- Also, chrysene, the Diels' hydrocarbon, and several tumorigenic PAHs such as benz[a]anthracene $B[a] A$, $\mathrm{DB}[a, h] \mathrm{A}$, and $\mathrm{B}[\mathrm{a}] \mathrm{P}$ are obviously related structurally to phenanthrene since each has one or more additional benzene rings fused to a phenanthrene structure.

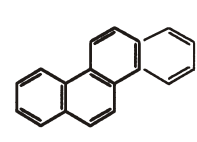

Chrysene

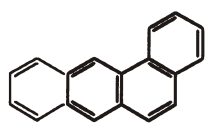

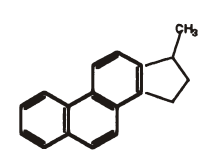

Diels' hydrocarbons
Benz[a]anthracene
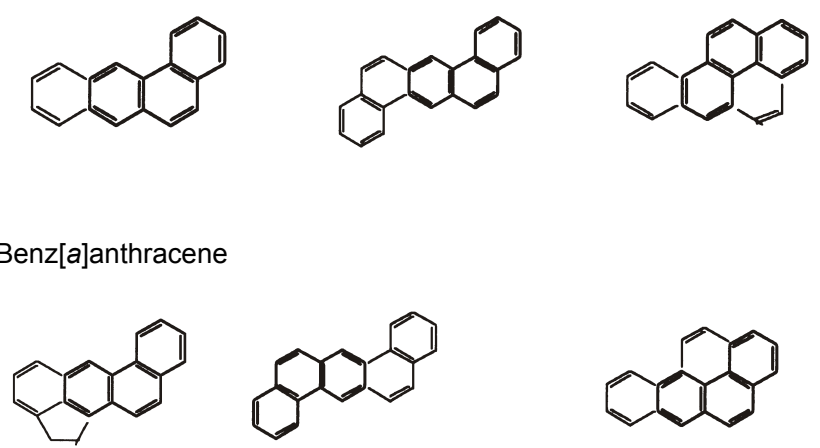

Cholanthrene

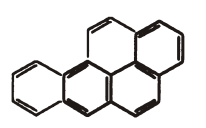

Benzo[a]pyrene 


\begin{tabular}{|c|c|}
\hline $\begin{array}{l}\begin{array}{l}\text { Roffo and the destructive distillate of tobacco, other related } \\
\text { research }\end{array} \\
\end{array}$ & Tobacco smoke and other related research \\
\hline $\begin{array}{l}\text { In } 1939, \text { Roffo reported the tumorigenicity to laboratory } \\
\text { animals of heated/oxidized "fat" }(851,852) \text {. }\end{array}$ & $\begin{array}{l}\text { Subsequent studies on heated fats essentially confirmed the } \\
\text { findings of Roffo, e.g., Peacock and Beck (853), Lane et al. } \\
\text { (854), but a study by Morris et al. }(855) \text { did not. } \\
\text { Fat components such as saturated hydrocarbons were shown } \\
\text { to yield tumorigens on heating, e.g., }(856,857) \text {. } \\
\text { Fat components such as high molecular weight aliphatic } \\
\text { esters and triglycerides yield tumorigens on heating (858, } \\
859,860) \text {. }\end{array}$ \\
\hline $\begin{array}{l}\text { Duplicating his tobacco destructive distillate procedure, Roffo } \\
\text { reported in } 1940 \text { that the destructive distillate from tea was } \\
\text { tumorigenic (861) as was the destructive distillate of Yerba } \\
\text { mate ( } 862) \text {. } \\
\text { Note: Yerba mate is a tea-like substance containing } \\
\text { xanthines and used for drink preparations, including toasted } \\
\text { Yerba mate used in drink preparation. }\end{array}$ & $\begin{array}{l}\text { In the late } 1950 \text { s, tumorigenic PAHs were reported in tea and } \\
\text { coffee by Kuratsune (863) and Kuratsune and Hueper (864). } \\
\text { In his } 1988 \text { review, Maga (865) summarized the identification } \\
\text { of numerous tumorigenic PAHs in foodstuffs and beverages, } \\
\text { including tea and coffee. }\end{array}$ \\
\hline $\begin{array}{l}\text { In } 1939, \text { Roffo reported that the destructive distillate from } \\
\text { coffee was tumorigenic to laboratory animals (866). }\end{array}$ & $\begin{array}{l}\text { In } 1939, \text { Widmark (867) reported that an extract of roasted } \\
\text { coffee was tumorigenic to laboratory animals. } \\
\text { With improved analytical technology available in the mid- } \\
1950 \text { s, B[a]P was identified in roast coffee by Fieser's } \\
\text { colleagues }(806,863,864) \text { but no B[a]P was identified by } \\
\text { them in cigarette "tar". }\end{array}$ \\
\hline
\end{tabular}

${ }^{a}$ Considered a major precursor of PAHs by Lam and by Wynder; demonstrated to be significant but less significant than phytosterols and terpenoids such as solanesol.

${ }^{b}$ Considered a major precursor of PAHs by Wynder and Wright; demonstrated to be major by Rodgman and Cook (843) and by Severson et al. (842).

${ }^{\circ}$ Considered a major precursor of PAHs by Wright; demonstrated to be major by Rodgman and Cook (843) and Severson et al. (842).

Later, in their lengthy 1964 review [see p. 259 in 807)] and 1967 book [see p. 94 in (808)], WYNDER and HOFFMANN essentially dismissed the research by ROFFO on a tobacco destructive distillate by tersely noting that the preparation by ROFFO of a tobacco "tar" for a carcinogenicity test involved the mere destructive distillation of tobacco, thus excluding the ROFFO investigations from any discussion of tobacco smoke.

Of course, most criticisms of ROFFO's research findings were based on his production of a tobacco tar by destructive distillation of tobacco, a process not considered comparable to the normal combustion of tobacco in a cigarette in the presence of air and its oxygen. It is indeed true that, during the cigarette puff, the oxygen level immediately ahead of the fire cone is depleted from the $\approx 21 \%$ level of air, but as NEWSOME and KEITH (809) demonstrated, the oxygen level is not $0 \%$ as it might be during destructive distillation. During the smoking process, the cigarette system has access to air (and its oxygen content) surrounding the cigarette both during and between puffs. Cigarette paper porosity also dictates some air flow through the cigarette paper during the puff.

In 1961, LARSON et al. [see pp. 422-424 in (810)] provided a critique of ROFFO's rabbit skin-painting studies with destructive distillates from tobaccos. ROFFO succeeded in producing tumors, many of which were described as carcinomas or epitheliomas, in the treated rabbits. Because of the small number of tumors generated in the ROFFO biological Studies, LARSON et al. considered that one must be very hesitant in placing too great weight on ROFFO's reiterated description of a solitary tumor.

ROFFO also claimed that his tobacco tar generated by destructive distillation was more tumorigenic than equivalent doses of coal tar, a finding at odds with those of many investigators, even those who compared the tumorigenicity in mice of various tobacco-tar preparations with that of equivalent doses of coal tar solutions (see Table 2). Despite the numerous criticisms of the information provided by ROFFO in his numerous publications issued in the 1930 s and early 1940 s on the destructive distillate from tobacco because of the purported lack of pertinence of such information to that derived from studies on tobacco-derived smoke, it is interesting to compare the results reported by ROFFO on the chemical and biological properties of the tobacco destructive distillate with those obtained post-1953 with tobacco smoke, particularly cigarette smoke. Also interesting is the comparison of the findings of RoFFO on the pyrolysis of several tobacco components with reports by other investigators. Such comparisons are also presented in Table 2.

Although many of the findings reported by RoFFO on the tobacco destructive distillate differ in degree from findings reported for tobacco smoke, many of the tobacco destructive distillate and tobacco smoke findings do parallel each other generally. As indicated in Table 2, two ROFFO findings do differ substantially from those reported by other investigators: 1) The relationship between the tumorigenicity of tobacco destructive distillate vs. coal tar tumorigenicity. 2) The similarity of the tumorigenicities of the destructive distillates from different tobacco types reported by $\operatorname{ROFFO}(70,71,72,73,825)$ vs. the difference in tumorigenicities of the mainstream smoke "tars" from various tobacco types reported by WYNDER and HOFFMANN (826). Recently, PROCTOR (868) summarized the contributions of ROFFO to our tobacco-pertinent knowledge and noted several of the investigators who were either supportive of or critical of his findings. 
Table 3. Some compounds applied to tobacco for treatment of diseases and pests prior to 1953

\begin{tabular}{|c|c|c|}
\hline CAS No. & Name & Reference \\
\hline \multicolumn{3}{|l|}{ Fungicides: } \\
\hline $50-00-0$ & Formaldehyde & 1945 Clayton (869) \\
\hline $7761-88-8$ & Silver nitrate & 1951 Garner (870) \\
\hline 7487-94-7 & Corrosive sublimate mercuric chloride $\mathrm{HgCl}_{2}$ & 1951 Garner (870) \\
\hline $71-43-2$ & Benzol $\{$ benzene $\}$ & 1951 Garner (870) \\
\hline $106-46-7$ & $p$-Dichlorobenzene & 1951 Garner (870) \\
\hline $1317-39-1$ & Copper oxide $\{$ yellow cuprocide $\}$ & 1951 Garner (870) \\
\hline $577-11-7$ & Sulfosuccinate & 1951 Garner (870) \\
\hline $14882-18-9$ & Bismuth subsalicylate & 1951 Garner (870) \\
\hline $14484-64-1$ & Ferric dimethyl-dithiocarbamate $\{$ Fermate $\}$ & 1951 Garner (870) \\
\hline $7758-98-7$ & Copper sulfate, anhydrous \{Bordeaux mixture\} & 1951 Garner (870) \\
\hline \multicolumn{3}{|l|}{ Pesticides: } \\
\hline $12002-03-8$ & Copper acetoarsenite $\{$ Paris Green $\}$ & 1951 Garner (870) \\
\hline $592-01-8$ & Calcium cyanide & 1951 Garner (870) \\
\hline $98-95-3$ & Nitrobenzene & 1951 Garner (870) \\
\hline \multirow[t]{3}{*}{$7784-40-9$} & Lead arsenate $\{$ Diplumbic lead arsenate $\}$ & 1901 Marlatt (209) \\
\hline & & 1908 Marlatt (210) \\
\hline & & 1951 Garner (870) \\
\hline $15096-52-3$ & $\begin{array}{l}\text { Sodium aluminofluoride } \\
\quad=\text { Sodium fluoaluminate }\{\text { Cryolite }\}\end{array}$ & 1951 Garner (870) \\
\hline $83-79-4$ & Rotenone & $\begin{array}{l}1937 \text { Chamberlin and Madden (871) } \\
1951 \text { Garner (870) }\end{array}$ \\
\hline $16893-85-9$ & Sodium fluosilicate & 1951 Garner (870) \\
\hline $107-49-3$ & Tetraethyl pyrophosphate & 1951 Garner (870) \\
\hline $56-38-2$ & Parathion dust & 1951 Garner (870) \\
\hline \multirow[t]{3}{*}{$8003-34-7$} & Pyrethrum & 1936 LaForge and Haller (872) \\
\hline & & 1944 LaForge and Barthel (873) \\
\hline & & 1951 Garner (870) \\
\hline $74-90-8$ & Hydrogen cyanide & 1951 Garner (870) \\
\hline $75-21-8$ & Ethylene oxide & 1951 Garner (870) \\
\hline $124-38-9$ & Carbon dioxide & 1951 Garner (870) \\
\hline $75-15-0$ & Carbon disulfide & 1951 Garner (870) \\
\hline \multirow[t]{3}{*}{$65-30-5$} & Nicotine sulfate & 1917 Moore and Graham (874) \\
\hline & & 1936 Busbey and McIndoo $(628,629)$ \\
\hline & & 1951 Garner (870) \\
\hline 68038-71-1 & Bacillus thuringiensis $\{$ B.t. $\} \quad\{$ Dipel [\} & 1924 Staudinger and Ruzicka (875) \\
\hline
\end{tabular}

\section{OTHER COMPOUNDS APPLIED TO TOBACCO BUT NOT SPECIFICALLY IDENTIFIED IN TOBACCO OR TOBACCO SMOKE PRIOR TO 1954}

In reviewing the tobacco and smoke literature prior to 1953 , two additional sets of compounds are provided for historical purposes. These include either 1) compounds applied on tobacco prior to 1953 for the treatment of disease (molds, spores, fungi, viruses, etc) and pests, or 2) compounds applied on tobacco as flavorants, casings and/or humectants. The listings are not complete as farmers did not always reliably report such usage for their crops and tobacco manufacturers rarely reported the composition of flavor additives, casings, and humectants used on tobacco prior to 1953. Table 3 and Table 4 are primarily of historical value as the chemicals applied to tobacco for disease control and the types of additives applied to tobacco have markedly changed over time. Most of the chemicals in Tables 3 and 4 are not listed in Table 1 because they were not isolated and confirmed to be present on tobacco samples that were analyzed in the articles reviewed. Nonetheless, these compounds were applied to tobacco and may have been present as residues in the case of the fungicides and pesticides or at larger levels in the case of flavorants and humectants. These compounds are listed in Tables 3 and 4.

\section{REFERENCES}

1. Kosak, A.I.: The composition of tobacco smoke; Experientia 10 (1954) 69-71.

2. Rodgman, A. and T.A. Perfetti: The chemical components of tobacco and tobacco smoke; CRC Press, Boca Raton, FL (2008).

3. Graham, E.A., E.L. Wynder, and A.B. Croninger: Cigarette smoking and cancer of the lung; Science 116 (1952) 521-522.

4. Wynder, E.L., E.A. Graham, and A.B. Croninger: Study on the experimental production of cancer with tobacco tar; Proc. Am. Assoc. Cancer Res. 1 (1953) 62-63.

5. Wynder, E.L., E.A. Graham, and A.B. Croninger: Experimental production of carcinoma with cigarette tar; Cancer Res. 13 (1953) 855-864.

6. Hoffmann, E., H.T. Schreus, and E. Zurhelle: Beobachtungen zur experimentellen Geschwulsterzeugung durch Teer verschiedener Herkunft und Paraffin [Observations on experimental tumour production by tars from different sources and paraffin]; Deut. Med. Wchnschr. 49 (1923) 633-634.

7. Helwig, F.C.: Influence of tobacco and other extracts on the epithelial cell; J. Kansas Med. Soc. 27 (1927) 37-40. 


\begin{tabular}{|c|c|c|}
\hline CAS No. & Name & Reference \\
\hline & Liquidambar $\{$ sap of the sweet gum tree\} & 1632 Diaz del Castillo (876) \\
\hline $\begin{array}{r}8008-94-4 \\
68916-91-6\end{array}$ & Licorice & 1951 Garner (877) \\
\hline $57-50-1$ & Sugar & 1951 Garner (877) \\
\hline $50-99-7$ & Glucose & 1951 Garner (877) \\
\hline $8013-17-0$ & Invert sugar & 1951 Garner (877) \\
\hline $8028-66-8$ & Honey & 1951 Garner (877) \\
\hline $9000-40-2$ & St. John's bread & 1951 Garner (877) \\
\hline $68476-78-8$ & Molasses & 1951 Garner (877) \\
\hline \multirow[t]{2}{*}{ 8024-04-2 } & Tonka bean & 1951 Garner (877) \\
\hline & Deer's tongue & 1951 Garner (877) \\
\hline $91-64-5$ & Coumarin & 1951 Garner (877) \\
\hline $90604-31-1$ & Rum & 1951 Garner (877) \\
\hline $91450-09-8$ & & \\
\hline $56-81-5$ & Glycerol & 1951 Garner (877) \\
\hline $111-46-6$ & Diethylene glycol & 1951 Garner (877) \\
\hline
\end{tabular}

8. Helwig, F.C.: The growth-producing effects of extracts of tobacco on mice; J. Am. Med. Assoc. 91 (1928) $150-151$.

9. Bogen, E. and R.N. Loomis: Tobacco tar: An experimental investigation of its alleged carcinogenic action; Am. J. Cancer 16 (1932) 1515-1521.

10. Cooper, E.A., F.W.M. Lamb, E. Sanders, and E.L. Hirst: The role of tobacco smoking in the production of cancer; J. Hyg. (London) 32 (1932) 293-300.

11. Campbell, J.A.: The effects of exhaust gases from internal combustion engines and of tobacco smoke upon mice, with special reference to incidence of tumours of the lung; Brit. J. Exptl. Pathol. 17 (1936) $146-158$.

12. Campbell, J.A.: Carcinogenic agents present in the atmosphere and incidence of primary tumours in mice; Brit. J. Exp. Pathol. 20 (1939) 122-132.

13. Schürch, O. and A. Winterstein: Experimentelle Beiträge zur Frage Tabak und Krebs [Experimental contribution to the question of tobacco and cancer]; Z . Krebsforsch. 46 (1937) 414-419.

14. Taki, S.: The carcinogenic activity of substances isolated from tobacco tar; Osaka Daigaku Igaku Zassi 36 (1937) 483-484. Cited by Kinosita, R.: Studies on carcinogenic chemical substances; Trans. Soc. Path. Japan 27 (1937) 665-727.

15. Sugiura, K.: Observations on animals painted with tobacco tar; Am. J. Cancer 38 (1940) 41-49.

16. Flory, C.M.: The production of tumors by tobacco tars; Cancer Res. 1 (1941) 262-276.

17. Flory, C.M.: Accíon cancerigena del alquitran de tabaco [Carcinogenic action of tobacco tar]; Bol. Inst. Med. Exptl. Estud. Cáncer 19 (1942) 317-319.

18. Shubik, P.: Personal communication to E.L. Wynder, see Footnote, Table 1 in: Wynder, E.L., E.A. Graham, and A.B. Croninger: Experimental production of carcinoma with cigarette tar; Cancer Res. 13 (1953) 855-864.

19. Wacker, L. and A. Schmincke: Experimentelle Untersuchungen zur kausalen Genese atypischer Epithelwucherungen [Experimental investigation of the causal genesis of atypical epithelial proliferation]; Münch. Med. Wchnschr. 58 (1911) 1680-1682.

20. Yamagiwa, K.: Collected papers on artificial production of cancer; Maruzen Company Ltd., Tokyo,
Japan (1965); Yamagiwa, K. and K. Ichikawa: Experimentelle Studie über die Pathogenese der Epitheliengeschwülste [Experimental study of the pathogenesis of epithelial tumors]; Tokyo Igakkai Zassi 15 (1915) 295-344; Experimental study of the pathogenesis of carcinomas; J. Cancer Res. 3 (1918) 1-29.

21. Yamagiwa, K. and K. Ichikawa: Experimental study of the pathogenesis of carcinomas; .J. Cancer Res. 3 (1918) 1-29, see Yamagiwa, K.: Collected papers on artificial production of cancer; Maruzen Company Ltd., Tokyo, Japan (1965).

22. Bradford, J.A., W.R. Harlan, and H.R. Hanmer: Nature of cigaret smoke: Technic of experimental smoking; Ind. Eng. Chem. 28 (1936) 836-839.

23. Wynder, E.L. and E.A. Graham: Tobacco smoking as a possible etiologic factor in bronchiogenic carcinoma: A study of 684 cases; J. Am. Med. Assoc. 143 (1950) 329-336.

24. Doll, R. and A.B. Hill: Smoking and carcinoma of the lung. Preliminary report; Brit. Med. J. 1950 (ii) 739-748.

25. Levin, M.L., H. Goldstein, and P.R. Gerhardt: Cancer and tobacco smoking: A preliminary report; J. Am. Med. Assoc. 143 (1950) 336-338.

26. Mills, C.A. and M.M. Porter: Tobacco smoking habits and cancer of the mouth and respiratory system; Cancer Res. 10 (1950) 539-542.

27. Schrek, R., L.A. Baker, G.P. Ballard, and S. Dolgoff: Tobacco smoking as an etiologic factor in disease; Cancer Res. 10 (1950) 49-58.

28. Doll, R. and A.B. Hill: A study of the aetiology of carcinoma of the lung; Brit. Med. J. 1952(ii) 1271-1286.

29. McConnell, B.B., K.C.T. Gordon, and T. Jones: Occupational and personal factors in the etiology of cancer of the lung; Lancet 1952(ii) 651-656.

30. Koulumies, M.: Smoking and pulmonary carcinoma; Acta Radiol. 39 (1953) 255-260.

31. Lickint, F.: Ätiologie und Prophylaxe des Lungenkrebses. 2. Statistische Voraussetzungen zur Klärung der Tabakrauchätiologie des Lungenkrebses [Etiology and prophylaxis of lung cancer. 2. Statistical assumptions for clarification of the tobacco smoke etiology of lung cancer]; Steinkopf (1953).

32. Sadowsky, D.A., A.G. Gilliam, and J. Cornfield: The 
statistical association between smoking and carcinoma of the lung; J. Natl. Cancer Inst. 13 (1953) 1237-1258.

33. Adler, I.: Primary malignant growths of the lungs and bronchi; Longmans, Green and Co., New York, NY (1912).

34. Tylecote, F.E.: Cancer of the lung; Lancet 1927 (ii) 256-257.

35. Lickint, F.: Der Bronchialkrebs der Raucher [Bronchial cancer of the smoker]; Münch. Med. Wchnschr. 82 (1935) 1232-1234.

36. Arkin, A. and D.H. Wagner: Primary carcinoma of the lung; J. Am. Med. Assoc. 106 (1936) 587-591.

37. Kennaway, N.M. and E.L. Kennaway: A study of the incidence of cancer of the lung and larynx; J. Hyg. 36 (1936)236-267.

38. Kennaway, E.L. and N.M. Kennaway: A further study of the incidence of cancer of the lung and larynx; Brit. J. Cancer 1 (1947) 260-298.

39. Roffo, A.H.: Der Tabak als krebserzeugendes Agens [Tobacco as a cancer-inducing agent]; Deut. Med. Wchnschr. 63 (1937) 1267-1271.

40. Müller, P.H.: Tabakmissbrauch und Lungencarcinom [Tobacco misuse and lung carcinoma]; Z. Krebsforsch. 49 (1939) 57-85.

41. Ochsner, A. and M. DeBakey: Surgical considerations of primary carcinoma of the lung; Surgery 8 (1940) 922-1023.

42. Ochsner, A. and M. DeBakey: Carcinoma of the lung; Arch. Surg. 42 (1941) 209-258.

43. Schairer, E. and E. Schöninger: Lungenkrebs und Tabakverbrauch [Lung cancer and tobacco consumption]; Z. Krebsforsch. 54 (1943) 261-269.

44. USPHS: Smoking and health: Report of the Advisory Committee to the Surgeon General of the Public Health Service; PHS Publ. No. 1103 (1964).

45. USPHS: Smoking and health. A report of the Surgeon General; DHEW Publ. No. (PHS) 79-50066 (1979).

46. USPHS: The health consequences of smoking. Cancer. A report of the Surgeon General; DHHS Publ. No. (PHS) 82-50179 (1982) 183-235.

47. Ochsner, A.: Primary pulmonary malignancy. Treatment by total pneumonectomy. Analysis of seventynine collected cases and presentation of seven personal cases; Surg. Gynecol. Obstr. 68 (1939) 435-451.

48. Ochsner, A.: Smoking and cancer: A doctor's report; J. Messner, New York, NY (1954).

49. Ochsner, A., M. DeBakey, and I.M. Richman: Bronchogenic carcinoma; Southern Surg. 14 (1948) 595-604.

50. Bradford, J.K., A. Ochsner, J. Blalock, and H.B. Hatch: Bronchial carcinoma: A problem in management; Ochsner Clin. Rept. 3(1) (1957) 1-16.

51. Ochsner, A., M. DeBakey, and J.L. Dixon: Primary pulmonary malignancy treated by resection. An analysis of 129 cases of carcinoma of the lung; Ann. Surg. 12 (1947) 522-539.

52. Ochsner, A., M. DeBakey, and J.L. Dixon: Primary carcinoma of the lung; J. Am. Med. Assoc. 135 (1947) 321-327.

53. Wenusch, A. and R. Schöller: Beiträge zur Kenntnis der Zusammensetzung des Zigarrenrauches [Contribution to the knowledge of the composition of cigar smoke]; Fachl. Mitt. Österr. Tabakregie 1933(2) $15-19$.

54. Wenusch, A. and R. Schöller: Myosmin und Sokratin
[Myosmine and socratine]; Fachl. Mitt. Österr. Tabakregie 1934(1) 5-9.

55. Wenusch, A. and R. Schöller: Über den Nachweis von Nebenalkaloiden im Tabak und Tabakrauch. Teil I [The detection of minor alkaloids in tobacco and tobacco smoke. Part I]; Fachl. Mitt. Österr. Tabakregie 1935(1) 11.

56. Wenusch, A. and R. Schöller: Über den Nachweis von Nebenalkaloiden im Tabak und Tabakrauch. Teil II [The detection of minor alkaloids in tobacco and tobacco smoke. Part II]; Fachl. Mitt. Österr. Tabakregie 1935(3) 2-7

57. Wenusch, A. and R. Schöller: Über das Schicksal des Nikotins beim Rauchen. Abbauprodukte des Nikotins [The fate of nicotine during smoking. Degradation products of nicotine]; Fachl. Mitt. Österr. Tabakregie 1936(1) 3-7.

58. Kuffner, F., K. Schick, and H. Bühn: Über die Sokratine, des Obelins, und andere Nebenalkaloide des Zigarettenrauches [About sokratine, obelin, and other secondary alkaloids in cigarette smoke]; Monatsh. Chem. 87 (1956) 749-762; Kuffner, F.: Über Alkaloide des Tabakrauch und die Konstitution des Nicotellins [About the alkaloids in tobacco smoke and the constitution of nicotellin]; $14^{\text {th }}$ Internat. Cong. Pure \& Appl. Chem., Zurich Switzerland (1955); Fachl. Mitt. Österr. Tabakregie 1956(1) 18-19.

59. Johnstone, R.A.W. and J.R. Plimmer: The chemical constituents of tobacco and tobacco smoke; Chem. Rev. 59 (1959) 885-936.

60. Vinzant, J.P.: Insecticidal residues and their effects on the smoking quality of cigar and cigarette tobaccos; $5^{\text {th }}$ Tobacco Chemists' Research Conference, Program Booklet and Abstracts, Vol. 5, Paper No. 4, 1951.

61. Cogbill, E.C. and M.E. Hobbs: The transfer of metallic constituents to the main-stream smoke; Tob. Sci. 1 (1957) 68-73.

62. Guthrie, F.E., C.B. McCants, and H.G. Small: Arsenic content of commercial tobacco, 1917-1958; Tob. Sci. 3 (1959) 62-64.

63. Larson, P.S., H.B. Haag, and H. Silvette: Tobacco: Experimental and clinical studies, Williams and Wilkins Company, Baltimore, MD, 1961.

64. Brasch, W. and C. Neuberg: Biochemische Umwandlung der Glutaminsäure in $n$-Buttersäure [Biochemical conversion of glutamic acid into butyric acid]; Biochem. Z. 13 (1908) 299-304. Cited by A. Wenusch in: Der Tabak. Seine Entstehung, Beschaffenheit und Zusammensetzung [Tobacco. Its quality, formation and composition], Arthur Geist, Bremen, Germany, 1939.

65. Brasch, W.: The bacterial degradation of the primary scission products of proteins; Biochem. Z. 18 (1909) 380-390. Cited by A. Wenusch in: Der Tabak. Seine Entstehung, Beschaffenheit und Zusammensetzung [Tobacco. Its quality, formation and composition], Arthur Geist, Bremen, Germany, 1939.

66. Neuberg, C. and J. Burkard: Über neue stickstofffreie Bestandteile des Tabakrauches [Some new nitrogenfree components of tobacco smoke]; Biochem. Z. 243 (1931) 472-484, see Chem. Abstr. 26 (1932) 2012.

67. Pfyl, B.: Zur Bestimmung des Nikotins im Tabakrauch. II [On the determination of nicotine in tobacco smoke. II]; Z. Untersuch. Lebensm. 66 (1933) 501-510. 
68. Neuberg, C. and M. Kobel: Living processes in fresh and dry tobacco leaves before and during fermentation; Biochem. Z. 179 (1926) 459-490.

69. Dixon, F.A. F.R. Darkis, F.A. Wolf, J.A. Hall, E.P. Jones, and P.M. Gross: Flue-cured tobacco: Natural aging of flue-cured cigarette tobaccos; Ind. Eng. Chem. 28 (1936) 180-189.

70. Roffo, A.H.: Unidad cancerígena de los alquitranes de diversos tipos de tabacos [Uniform carcinogenicity of the tars from different tobacco types]; Bol. Inst. Med. Exptl. Estud. Cáncer 15 (1939) 349-406, see Chem. Abstr. 33 (1939) 5906 .

71. Roffo, A.H.: Unidad cancerígena de los alquitranes de diversos tipos de tabacos [Uniform carcinogenicity of the tars from different tobacco types]; Prensa Méd. Argent. 26 (1939) 721-737, see Chem. Abstr. 34 (1940) $7120^{4}$

72. Roffo, A.H.: Krebserzeugende Einheit der verschiedener Tabakteere [Uniformity of cancercausing properties of different tobacco tars]; Deut. Med. Wchnschr. 65 (1939) 963-967, see Chem. Abstr. 33 (1939) $9419^{7}$.

73. Roffo, A.H.: Le goudron de tabac cancérigène. Unité cancérigène des goudrons de diverse typos de tabac [Carcinogenic tar from tobacco. Uniform carcinogenicity of tars from different tobacco types]; Acta Unio Internat. Contra Cancrum 4 (1939) 755-763.

74. Zeise, W.C.: Untersuchungen der bei der trockenen Destillation von Tabak entstehenden Produkte und der chemischen Verbindungen des Tabakrauches [Investigation of the products of dry distillation of tobacco and the chemical constituents of tobacco smoke]; Ann. Chem. Pharm. 47 (1843) 212-225.

75. Vohl, H. and H. Eulenberg: The physiological action of tobacco used as a narcotic, with special reference to the constituents of tobacco smoke; Arch. Pharm. 147 (1871) 130-167, see abstr. in J. Chem. Soc. (1871) 1075-1078.

76. Vohl, H. and H. Eulenberg: The toxicological nature of tobacco with particular reference to the chemical composition of smoke; Viertel. Gerich. Offent. Med. 14 (1871) 249-290; abstr. in J. Chem. Soc. (1871) 1075-1078.

77. Abeles, M. and L. Paschkis: Beiträge zur Kenntnis des Tabakrauches [Contribution to the knowledge of tobacco smoke]; Arch. Hyg. 14 (1892) 209-215.

78. Gabelya, Y.O. and G.I. Kipriyanov: The dry distillation of tobacco; Ukrainskii Khem. Zhur. 4 (1929) 45-63, see Chem. Abstr. 24 (1930) 916.

79. Bradford, J.A., E.S. Harlow, W.R. Harlan, and H.R. Hanmer: Nature of cigaret smoke: Volatile bases and acids; Ind. Eng. Chem. 29 (1937) 45-50.

80. Peterson, L.A.: Evaluation of the constituents of tobacco smoke; Report of Proceedings of the Research Conference on the Chemistry of Tobacco, Pennsylvania (1950) 9-10.

81. Garner, W.W.: The production of tobacco; Revised $1^{\text {st }}$ Ed., The Blakiston Co., New York, NY (1951) pp. $1-520$.

82. James, A.T. and A.J.P. Martin: Gas-liquid partition chromatography: The separation and micro-estimation of volatile fatty acids from formic acid to dodecanoic acid; J. Biochem. 50 (1952) 679-690.

83. Takayama, J.: On Japanese tea and tobacco; Chem. News 50 (1884) 299-301.
84. Garner, W.W.: Relation of nicotine to quality of tobacco; Bureau of Plant Industry, Bulletin 141 (1909).

85. Shmuk, A.A.: An investigation of the tars and aromatic substances of tobacco; Institute of Experimental Tobacco Growing, No. 20 (1924) 5.

86. Balabucha-Popzova, B.: Pectin substances of tobacco; U.S.S.R. State Inst. Tobacco Invest. Bull. 59 (1929) 1-25, see Chem. Abstr. 24 (1930) 4581.

87. Shmuk, A.A.: Investigations of the tobacco acids (First Report); U.S.S.R. State Inst. Tobacco Invest. Bull. 50 (1929) 1-10, see Chem. Abstr. 23 (1929) 4771.

88. Yamafuji, K.: The chemical constituents of tobacco leaves. I. Organic bases and acids; J. Agr. Chem. Soc. Japan 7 (1931) 121-123, see Chem. Abstr. 25 (1931) 3377.

89. Koenig, P.: Chemische und physikalische Unterschiede schwerer und leichter Tabake [Chemical and physical differences between strong and mild tobaccos]; Z. Untersuch. Lebensm. 70 (1935) 26-33.

90. Sabetay, S., L. Trabaud, and H.F. Emmanuel: Constituents of the concrete oil of tobacco leaves (Nicotiana tabacum); Compt. Rend. 213 (1941) 321-323; Chim. Ind. (Paris) 46 (1941) 429, see Chem. Abstr. 37 (1943) 1831.

91. Wenusch, A. and R. Schöller: Über die Menge wasserdampfflüchtiger Säuren und Basen der festflüssigen Bestandteile des Tabakrauches [Amount of steam-volatile acids and bases in the nonvolatile constituents of tobacco smoke]; Z. Untersuch. Lebensm. 74 (1937) 408-411.

92. Haag, H.B.: Chemical and pharmacologic observations on nicotine and tobacco smoke; The Merck Report (October, 1940) pp. 25-29.

93. Shmuk, A.A. and N. Piatnicki: Acids of tobacco. II; J. Assoc. Off. Agr. Chem. 69 (1930) 19-26, see Chem. Abstr. 25 (1931) 3124.

94. Wada, E. and Y. Kobashi: Chemical constituents of tobacco. IV. Determination of organic acids in tobacco leaves and isolation of organic acids by paper chromatography; J. Agr. Chem. Soc. Japan 27 (1953) 561-564, see Chem. Abstr. 50 (1956) 17342e.

95. Venkatarao, C., M. Narasingarao, and A. Venkateswarulu: Fatty acid composition of tobacco seed oil; J. Indian Chem. Soc. 20 (1943) 374, 376.

96. Roberts, E.A.H. and D.J. Wood: The polyphenols and amino acids of tobacco leaf; Arch. Biochem. 33 (1951) 299-303.

97. Frankenburg, W.G. and A.M. Gottscho: Nitrogen compounds in fermented cigar leaves; Ind. Eng. Chem. 44 (1952) 301-305.

98. Pearse, H.L. and L. Novellie: South African tobacco. I. Preliminary identification of amino acids and other constituents; J. Sci. Food Agr. 4 (1953) 108-112, see Chem. Abstr. 47 (1953) 5638e.

99. Zacharius, R. and W.E. Frankenburg: Some observations on proteins and amino acids in cigar tobacco leaves; $7^{\text {th }}$ Tobacco Chemists' Research Conference, Program Booklet and Abstracts, Vol. 7, Paper No. 8, 1953, p. 5.

100. Garner, W.W., G.W. Bacon, and C.L. Foubert: Research studies on the curing of leaf tobacco; Bulletin of the U.S. Department of Agriculture, No. 79 (1914).

101. Vickery, H.B. and G.W. Pucher: Chemical investigations of the tobacco plant. I. A preliminary study of 
the non-volatile organic acids of tobacco leaves; Conn. Agr. Exp. Station Bull. 323 (1931).

102. Pucher, G.W. and H.B. Vickery: The katabolism of the non-volatile organic acids of tobacco leaves during curing; Physiology 19 (1933) 623-626.

103. Drobkov, A.A.: The influence of the radioactive elements uranium, radium, thorium, and actinium on the yield of plants; Compt. Rend. Acad. Sci. U.S.S.R. 17 (1937) 229-232, see Chem. Abstr. 32 (1937) 2569.

104. Ross, J.H.: Infrared spectra for analysis of aldehyde and ketone 2,4-dinitrophenylhydrazones; Anal. Chem. 25 (1953) 1288-1303.

105. Shmuk, A.A. and M. Kolesnik: The reaction of tobacco smoke in connection with the quality of tobacco; Narkom. SSSR-Soyuztabak Gosudar. Inst. Tabak Bull. 80 (1931) 45-52.

106. Wenusch, A.: Die Alkaloide des Tabakrauches [The alkaloidal compounds of tobacco smoke]; in: Wenusch, A.: Der Tabak. Seine Entstehung, Beschaffenheit und Zusammensetzung [Tobacco. Its quality, formation, and composition], Arthur Geist, Bremen, Germany (1939) pp. 75-80.

107. Latimer, P.H.: Determination of alkaloids in tobacco smoke by paper chromatography; $7^{\text {th }}$ Tobacco Chemists' Research Conference, Program Booklet and Abstracts, Vol. 7, Paper No. 4, 1953, p. 4.

108. Pictet, A. and A. Rotschy: Über neue Alkaloide des Tabaks [New alkaloids of tobacco]; Ber. Dtsch. Chem. Ges. 34 (1901) 696-704.

109. Pictet, A. and A. Rotschy: Sur trois nouveaux alcaloides du tabac [On three new alkaloids of tobacco]; Compt. Rend. 132 (1901) 971-972.

110. Pictet, A. and G. Court: Einige neue Pflanzenalkaloide [Some new vegetable alkaloids]; Ber. Dtsch. Chem. Ges. 40 (1908) 3771-3783, see Chem. Abstr. 2 (1908) $115-6$.

111. Ehrenstein, M.: Zur Kenntnis der Tabakalkaloide [On the knowledge of the alkaloids of tobacco] Ber. Dtsch. Pharm. Ges. 269 (1931) 627-659, also see Chem. Zeit. 52 (1928) 755, Ber. Dtsch. Pharm. Ges. 268 (1930) 430.

112. Marion, L.: Les alcaloides du tabac [Tobacco alkaloids]; Rev. Trimest. Canada 24 (1938) 170-184.

113.Späth, E. and F. Kuffner: Die Tabakalkaloide [Tobacco alkaloids]; Forschr. Chem. Org. Naturstoffe 2 (1939) 248-300.

114. Jackson, K.E.: Alkaloids of tobacco; Chem. Rev. 29 (1941) 123-197.

115. Frankenburg, W.G.: Determination of alkaloids and alkaloid transformation products; $2^{\text {nd }}$ Tobacco Chemists' Research Conference, Program Booklet and Abstracts, Vol. 2, Paper No. 2, 1948.

116. Badgett, C.O., A. Eisner, and H.A. Whalens: Distribution of pyridine alkaloids in the system buffertert-amyl alcohol; J. Am. Chem. Soc. 74 (1952) 4096-4098.

117. Tso, T.C. and R.N. Jeffrey: Paper chromatography of alkaloids and their transformation products in Maryland tobacco; Arch. Biochem. Biophys. 43 (1953) 269-285, see Chem. Abstr. 47 (1953) 9430f.

118. Bailey, P.C.: The quantitative determination of some constituents of tobacco smoke; Master's Thesis, Duke University (1952) pp. 1-40.

119. Eisenmenger, W.S.: Toxicity of aluminum for plants; Mass. Agr. Exp. Sta. Ann. Rpt. Bul. 293 (1932) 11-12.
120. McMurtrey, J.E. and W.O. Robinson: Neglected soil constituents that affect plant and animal development; U.S. Dept. Agr. Yearbook (1938) 807-829.

121. Morgan, M.F. and O.E. Street: Malnutrition symptoms due to deficiencies or excesses of plant food substances in tobacco; Connecticut Agr. Sta. Bull. 410 (1938) 393-406.

122.LeCompte, S.B.: Studies on black tobacco. III. Statistical analysis of a field crop; Conn. Sta. Bull. 448 (1944) 114-117.

123. Vogel, A.: Wassergehalt und ammonalkalische Verbrennungsprodukte des Tabaks [The water content and ammoniacal combustion products of tobacco]; Neues Repert. Pharm. 6 (1857), 1-3.

124.Vogel, A.: Über ammonalkalische Verbrennungsprodukte des Tabaks (Fortsetzung) [The ammoniacal combustion products of tobacco (continuation)]; Neues Repert. Pharm. 6 (1857) 153-156.

125. Vogel, A.: Über die ammonalkalische Verbrennungsprodukte und den Aschegehalt eniger Tabakarten [On the ammoniacal burning products and the ash content of certain tobacco types]; Dingler's Polytech. J. 148 (1858) 228-231.

126. Périgord, L.: La fumée du tabac [Tobacco smoke]; Thesis, Paris (1879) pp. 1-49.

127.LeBon, G.: La fumée du tabac [Tobacco smoke]; $2^{\text {nd }}$ Edition, Paris, France, 1880.

128. Thoms, H.: Chemische Untersuchungen des Tabakrauches [The chemical examination of tobacco smoke]; Chem. Ztg. 23 (1899) 852-854.

129. Thoms, H.: Über die Rauchprodukte des Tabaks [The smoke products of tobacco]; Ber. Deut. Pharm. Gesellschaft. 10 (1900) 19-31.

130. Pontag, J.J.: Investigation of Russian tobacco and cigarette smoke; Chem. Ztg. 26 (1902) 359.

131. Pontag, J.J.: Investigation of Russian smoking tobacco and cigarette smoke; Z. Untersuch. Nahrungs- und Genussmitt. Gebrauchsgegenstande 6(1903) 673-691.

132. Biederbeck, J.: Neue Untersuchungen über die Absorption von Nikotin und Ammoniak aus Luftmischung und dem Zigaretterauch [New investigation of the absorption of nicotine and ammonia from an air mixture and from cigarette smoke]; Dissertation, Würzburg (1908) pp. 1-24.

133. Lee, W.E.: The action of tobacco smoke, with special reference to arterial pressure and degeneration; Quart. J. Exp. Physiol. 1 (1908) 335-358.

134.Lehmann, K.B.: Untersuchungen über das Tabakrauchen [Investigations of tobacco smoke]; Münch. Med. Wchnschr. 55 (1908) 723-725.

135.Lehmann, K.B.: Chemische und toxikologische Studien über Tabak, Tabakrauch und das Tabakrauchen [Chemical and toxicological studies on tobacco, tobacco smoke and tobacco smoking]; Arch. Hyg. 68 (1909) 319-420.

136. Toth, J. and J. Krampera: Data on the amount of ammonia, pyridine bases, and nicotine in tobacco smoke; Kiserletugyi Koslemenyek 13 (1910) 617-622.

137. Vaubel, W.: Der Ammoniakgehalt des Tabakrauches [The ammonia content of tobacco smoke]; Chem. Zeit. 35 (1911) 1331-1332.

138. Anonymous: The toxic factor in tobacco; Lancet 1912 (ii) $547-548$.

139. Anonymous: The toxic factor in tobacco; Lancet 1912 
(ii) 944-947.

140. Shmuk, A.A.: Tobacco alkaloids; Works of the Scientific Research Institute for Special and Intensive Cultures at the Kuban Agricultural Institute, GIT (State Publishing House), Krasnodar, No. 44, 1928.

141. Bogen, E.: The composition of cigarets and cigaret smoke; J. Am. Med. Assoc. 93 (1929) 1110-1114.

142. Koperina, A.W.: Untersuchung der Stickstoffhältigen Verbindungen des Tabakrauches [Investigation of nitrogenous compounds in tobacco smoke]; Biochem. Z. 219 (1930) 258-276.

143. Gavrilov, N.J. and A.V. Koperina: Tabakchemie. IV. Analyse des Tabakrauches [Research on tobacco chemistry. IV. Analysis of tobacco smoke]; Biochem. Z. 231 (1931) 25-32.

144. Haley, D.E., C.O. Jensen, and O. Olson: A study of the ammonia content of cigar smoke; Plant Physiol. 6 (1931) 183-187.

145. Barta, L. and E. Toole: Über den Nikotin- und Ammoniakgehalt des Cigaretten-Tabakrauches [The nicotine and ammonia content of cigarette tobacco smoke]; Angew. Chem. 45 (1932) 43.

146. Barta, L. and E. Toole: Über den Nikotin- und Ammoniakgehalt des Cigaretten- Tabakrauches [The nicotine and ammonia content of cigarette tobacco smoke; Angew. Chem. 46 (1932) 671-673.

147. McNally, W.D.: The tar in cigarette smoke and its possible effects; Am. J. Cancer 16 (1932) 1502-1514.

148. Pfyl, B.: Zur Bestimmung des Nikotins im Tabakrauch. III [On the determination of nicotine in tobacco smoke. III]; Z. Untersuch. Lebensm. 66 (1933) 510-524.

149. Barta, L.: Über die Bestimmung des Ammoniaks im Tabakrauch [The determination of ammonia in tobacco smoke]; Angew. Chem. 47 (1934) 215-216.

150. Bogen, E.: Irritant factors in tobacco smoke; Cal. West. Med. 45 (1936) 342-346.

151. Preiss, W.: Zur Kenntnis des Rauchens [Information on smoking]; Z. Untersuch. Lebensm. 72 (1936) 196-212.

152. Preiss, W.: Zur Bestimmung von Nikotin, Ammoniak, Pyridinbasen und Teer im Tabakrauch und über die Wirksamkeit einiger Entnikotinisierungsspritzen [The determination of nicotine, ammonia, pyridine bases and tar in tobacco smoke and the efficiency of several denicotinization systems]; Pharm. Zentralhalle 77 (1936) 437-458.

153. Müller-Thurgen, H.: Über das Verhalten von Stärke und Zucker in reifenden und trocknenden Tabaksblättern [Starch and sugar behavior in ripening and drying tobacco leaves]; Landwirtschaftliche Jahrbücher 14 (1885) 485-512.

154.Behrens, T.: Weitere Beiträge zur Kenntnis der Tabakpflanze [Further contribution to the knowledge of the tobacco plant]; Landwirtschaftlichen VersuchsStationen 43 (1894) 271-301.

155. Smirnov, A.I. and V.P. Izvoshtshikov: Curing of yellow tobacco. II. The change in composition of nitrogenous groups of tobacco during the starving period; U.S.S.R. State Inst. for Tobacco Invest., Krasnodar, Bull. 71 (1930).

156. Preiss, W.: Bestimmung des Ammoniaks neben Pyridinbasen im Tabak und Tabakrauch [Determination of ammonia in the presence of pyridine bases in tobacco and tobacco smoke; Z. Untersuch.
Lebensm. 72 (1936) 189-196.

157. Fromm, F.: Die Bestimmung des Pyrrols im Tabak [Determination of pyrrole in tobacco]; Österr. Chemikerztg. 40 (1937) 434-437.

158. Gaertner, K.: Evaluation of tobacco on the basis of its chemical composition. II; Magyar Chem. Folyoirat 45 (1939) 19-30, see Chem. Abstr. 33 (1939) 7485.

159. Molinari, E.: Austria and the science of tobacco; Rev. Internat. Tab. 25 (1950) 195, see Chem. Abstr. 45 (1951) 3126g.

160. Hough, L., J.K.N. Jones, and E.L. Richards: The action of ammonia on glucose, maltose and lactose; J. Chem. Soc. (1952) 3854-3857.

161. Jensen, C.O.: Cigar tobaccos, chemical changes that occur during curing; Ind. Eng. Chem. 44 (1952) 306-309.

162. Dittmar, H.: Untersuchungen am Hauptrauch Iranischer Tabakfabrikate. I [Study of the mainstream smoke of Iranian tobacco products. I]; Pharm. Zentralhalle 80 (1939) 67-71.

163. Dittmar, H.: Untersuchungen am Hauptrauch Iranischer Tabakfabrikate. II [Study of the mainstream smoke of Iranian tobacco products. II]; Pharm. Zentralhalle 80 (1939) 259-263.

164. Dittmar, H.: Untersuchungen am Hauptrauch Iranischer Tabakfabrikate. III [Study of the mainstream smoke of Iranian tobacco products. III]; Pharm. Zentralhalle 80 (1939) 457-462.

165. Shmuk, A.A.: The taste quality and chemical composition of tobacco; Proceedings of the Academy of Sciences of the USSR, Biological Sciences Section, 1939, p. 955.

166. Pyriki, C.: Untersuchungen über den Rauch von Zigaretten [Investigation of the smoke from cigarettes]; Z. Untersuch. Lebensm. 88 (1948) 254-269, see Chem. Abstr. 42 (1948) 8425.

167.Larson, P.S.: Metabolism of nicotine and nature of tobacco smoke irritants; Ind. Eng. Chem. 44 (1952) 279-283.

168. Cuvelier, B.V.J.: Einfluss der Ammoniumsalze auf die Löslichkeit des Nikotins [Influence of ammonium salts on the solubility of nicotine]; Z. Anal. Chem. 105 (1936) 325-328.

169. Späth, E. and E. Zajic: Über neue Tabakalkaloide. VIII. Über Tabakbasen und Bemerkungen zur Kenntnis des Rhöadins, des L-Peganins und des Ammoresinols [On new tobacco alkaloids. VIII. On tobacco bases and observation on the presence of rhoeadin, $L$-peganin and ammoresinol]; Ber. Dtsch. Chem. Ges. 69 (1936) 2448-2452, see Chem. Abstr. 31 (1937) 1029.

170. Oosthuizen, J.D. and O.M. Shedd: Enzymes of the tobacco plant; J. Am. Chem. Soc. 35 (1913) 1289-1309, see Chem. Abstr. 7 (1913) 3608.

171. Matsusima, H.: Enzymes of fresh tobacco leaves; J. Taikoku Soc. Agr. Forestry 1 (1937) 271-280.

172. Ward, G.M.: Physiological studies with the tobacco plant; Can. Dept. Agr. Tech. Bull. 37 (1942).

173. Garner, W.W.: The production of tobacco; The Blakiston Company, Philadelphia, PA, 1946.

174. Nakai, T. and Y. Inaba: Fundamental studies of tobacco enzymes; J. Agr. Chem. Soc. Japan, 24 (1951) 105-110, see Chem. Abstr. 45 (1951) 6699.

175. Barrett, R.E., R.F. Robinson, and K.S. Chester: A study of the enzymes in cigarette tobacco; Battelle 
Memorial Institute (1953) 1-12.

176. Henry, T.A.: The plant alkaloids; $3^{\text {rd }}$ Edition, J.A. Churchill Ltd., London, (1939) p. 42.

177. Wenusch, A. and R. Schöller: Über das Auftreten anderer Alkaloide neben dem Nikotin im Tabak [The occurrence of alkaloids other than nicotine in tobacco]; Pharm. Zentralhalle 77 (1936) 108.

178. Roffo, A.H.: Krebserzeugendes Benzpyren gewonnen aus Tabakteer [Cancer-causing benzpyrene obtained from tobacco tar]; Z. Krebsforsch. 49 (1939) 588-597, see Biol. Abstr. 14 (1939) 16122.

179. Cooper, R.L. and A.J. Lindsey: The presence of polynuclear hydrocarbons in cigarette smoke; Chem. and Ind. (London) (1953) 1205.

180. Heffer, W. and Sons: Bibliography of the more important heavy metals occurring in food and biological materials; Cambridge, England (1934) pp. $1-30$.

181. Gabelya, Y.O. and G.I. Kipriyanov: The content of pectin in tobacco; Ukrainskii Khem. Zhur. 4 (1929) $37-43$.

182. Vickery, H.B., G.W. Pucher, A.J. Wakeham, and C.S. Leavensworth: Chemical investigations of the tobacco plant. I. Chemical changes that occur in leaves of Connecticut shade-grown tobacco during early stages of curing; Carnegie Inst. Wash. Pub. 445 (1933).

183. Vickery, H.B., G.W. Pucher, C.S. Leavensworth, and A.J. Wakeham: Chemical investigations of the tobacco plant. V. Chemical changes that occur during growth; Conn. Agr. Expt. Sta. Bull. 374 (1935).

184. Leitch, A. and E.L. Kennaway: Experimental production of cancer by arsenic; Brit. Med. J. (1922)(2) 1109.

185. Remington, R.E.: A hitherto unsuspected source of arsenic in the human environment; J. Am. Chem. Soc. 49 (1927) 1410-1416.

186. Gross, C.R. and O.A. Nelson: Arsenic in tobacco smoke; Am. J. Publ. Hlth. 24 (1934) 36-42.

187. Bastedo, W.A.: What the physician should know about tobacco; Medical Record 141 (1935) 553-555.

188. Thomas, M.D. and T.R. Collier: Concentration of arsenic in tobacco smoke determined by rapid titrimetric method; J. Ind. Hyg. Toxicol. 27 (1945) 201-206.

189. Griffon, H. and J. Delga: On the arsenic content of several kinds of commercial tobaccos. Fate of arsenic during combustion; Ann. Pharm. Franc. 5 (1947) 343-352.

190. Daff, M.E. and E.L. Kennaway: The arsenic content of tobacco and of tobacco smoke; Brit. J. Cancer 4 (1950) 173-182.

191.Daff, M.E. and E.L. Kennaway: Arsenic in tobacco; Brit. Emp. Cancer Camp., Ann. Rpt. 28 (1950) 95-99.

192.Daff, M.E., R. Doll, and E.L. Kennaway: Cancer of the lung in relation to tobacco; Brit. J. Cancer 5 (1951) $1-20$.

193. Goulden, F., E.L. Kennaway, and M.E. Urquhart: Arsenic in the suspended matter of town air; Brit. J. Cancer 6 (1952) 1-7.

194. Monnet, R. and O. DuPont: L'arsenic dans les tabacs d'Algérie: Tabacs à fumer et à macher [Arsenic in Algerian tobaccos: Tobaccos to smoke and to chew]; Bull. Algérien Cancer 6(17) (1953) 19-24.

195. Boening, G.: Arsen im Tabak [Arsenic in tobacco]; Chem. Ztg. 29 (1905) 183-184.
196. Popp, H.: Über das Vorkommen von Arsen im Tabak [Occurrence of arsenic in tobacco]; Z. Angew. Chem. Zent. Tech. Chem. 41 (1928) 838-839.

197. Popp, H.: Über das Vorkommen von Arsen im Tabak [The occurrence of arsenic in tobacco]; Chem. Z. 52 (1928) 501.

198. Carey, F.P., G. Blodgett, and H. S. Satterlee: Preparation of samples for determination of arsenic; Ind. Eng. Chem. (Anal. Ed.) 6 (1934) 327-330.

199. McMurtrey, J.E.: Boron deficiency in tobacco under field conditions; Am. Soc. Agron. 27 (1935) 271-273.

200. McMurtrey, J.E.: Symptoms on field-grown tobacco characteristic of the deficient supply of each of several essential chemical elements; U.S. Dept. Agr. Tech. Bull. 612 (1938).

201. Barksdale, E.E.: Arsenical exfoliative dermatitis with tobacco being the probable source of the etiological agent. Preliminary report; Virginia Med. Month. 66 (1939) 660-662.

202. Barksdale, E.E.: Arsenical dermatitis from tobacco; Virginia Med. Month. 67 (1940) 393-397.

203. McMurtrey, J.E.: Plant-nutrient deficiency in tobacco, in Hunger signs in crops, Am. Soc. Agron. Am. Fertilizer Assoc., Washington, DC (1941) 15-54.

204. Vucetich, D.C. and R. Carratala: Presence of arsenic in tobacco; Experimental investigation; Revista Asoc. Med. Argentina 56 (1942).

205. Vincent, C.L.: Vegetable and small fruit growing in toxic ex-orchard soils of central Washington; Wash. Agric. Expt. Bull. 437 (1944) 31.

206. Oliver, J.H.: Arsenic and tobacco; Lancet 1951(2) 266.

207.Bunce, E.H.: Arsenic and lead in cigarette brands; Lancet 263 (1952) 1082.

208. Wolff, W.A., E.G. Purdom and J.A. Isenhower: Radioisotopes in an experimental study of smoking; $7^{\text {th }}$ Tobacco Chemists' Research Conference, Program Booklet and Abstracts, Vol. 7, Paper No. 12, 1953, p. 8.

209. Marlatt, C.L.: Important insecticides: Directions for their preparation and use; Farmer's Bulletin, No. 117, USDA, Washington, DC (1901).

210. Marlatt, C.L.: Important insecticides: Directions for their preparation and use; $2^{\text {nd }}$ revision of Farmer's Bulletin No. 127, USDA, Washington, DC (1908).

211. Maton, J.: The influence of manganese ions on the synthesis of ascorbic acid in the green parts of higher plants; Biol. Jaarboek Koninkl. Natuurn. Genootschap Dodonaea Gent. 14 (1947) 109-115, see Chem. Abstr. 44 (1950) 2091h.

212.Lona, F. and F. Porzio-Giovanola: Ascorbic acid in tobacco; Nuova Giorna Bot. Ital. 58 (1951) 462, see Chem. Abstr. 47 (1953) 11365g.

213. Tombesi, L.: Ascorbic acid in tobacco; Il Tabacco 55 (1951) $15,53$.

214. Vickery, H.B., G.W. Pucher, A.J. Wakeham, and C. S. Leavensworth: The metabolism of amides in green plants. I. The amides of the tobacco leaf; J. Biol. Chem. 119 (1937) 369-382.

215. Ikeda, S.: Contribution to the study of tobacco smoke; Sci. Papers, Inst. Phys. Chem. Res. (Tokyo) 42 (1947) 80.

216. McHargue, J.S.: The occurrence of barium in tobacco and other plants; Jour. Amer. Chem. Soc. 35(6) (1913) 326-334.

217. Traetta-Mosca, F.: Cesium in Italian burley; Gazz. 
Chim. Ital. 43 (1913) 437; Titanium and rare metals in tobacco ash; Gazz. Chim. Ital. 43 (1913) 437-440.

218. Artis, B. and H.L. Maxwell: Barium in tobacco and other plants; Chem. News 114 No. 2959 (1916) 62-63.

219. Knight, N.: Barium in tobacco and other plants; Proc. Iowa Acad. Sci. 23 (1916) 26-29.

220. Headden, W.P.: Titanium, barium, strontium, and lithium in certain plants; Colorado Sta. Bull. 267 (1921) 3-20.

221. Nito, T. and E. Kitamura: Chemical constituents of tobacco leaves. I. Organic bases in the flue-cured tobacco; J. Agr. Chem. Soc. Japan 10 (1934) 424-426, see Chem. Abstr. 28 (1934) 4836.

222. Frankenburg, W.G.: Chemical changes in the harvested tobacco leaf, in Advances in Enzymology, Vol. VI, Interscience, New York, NY (1946) 308-387, see 331 .

223. Kissling, R.: Der Tabak im Lichte der neusten naturwissenschaftlichen Forschungen; kurz gefasstes Handbuch der Tabakkunde [Tobacco in the light of the newest natural science research; abridged manual of tobacco science]; Paul Parey, Berlin, Germany (1893) 278.

224. Molinari, E.: Über das Auftreten von Phenolen im Tabakrauch [The occurrence of phenols in tobacco smoke]; Fachl.. Mitt. Österr. Tabakregie 1936(3) 14-16.

225. Volgunov, G.P.: The decrease of the oxidative processes in tobacco after drying; Tabak 13 (1952) 15-17, see abstr. in Chem. Zentr. (1955) 7572 (Aug. $10)$.

226. Wenusch, A.: Über die Zusammensetzung des Tabakrauches [On the composition of tobacco smoke]; Österr. Chem. Z. 42 (1939) 226-231, see Chem. Abstr. 33 (1939) 6524.

227. Riemenschneider, R.W., R.M. Speck, and E.G. Beinhart: Analysis and fatty acid composition of tobacco-seed oils; Oil\&Soap 22 (1945) 120-122.

228. Best, R.J.: Fluorescent substances in plants: III. Distribution of scopoletin in tobacco plants and some hypothesis on its part in metabolism; Australian J. Exptl. Biol. Med. Sci. 26 (1948) 223-230, see Chem. Abstr. 42 (1948) 7842c.

229. Johanson, R.: Differential fluorescence in identification of trashy leaf; Nature 171 (1953) 753-754.

230. Kurilo, M.E.: Tobacco glucoside; Sborn. Rabot Khim. Tabak. Bull .125 (1935) 117-131.

231.Kurilo, M.E.: Le glucoside isoquercitrin dans les feuilles de tabac à fumer [The glucoside isoquercitrin in the leaves of smoking tobacco]; J. Pharm. Chim. 26 (1937) 445-459.

232. Howard, W.L., T.B. Gage, and S.H. Wender: The isolation of isoquercitrin from air-dried tobacco; Arch. Biochem. 25 (1950) 74-78.

233. Neuberg, C. and M. Kobel: Über die Isolierung von Rutin aus Tabak [Isolation of rutin from tobacco]; Z. Untersuch. Lebensm. 72 (1936) 113-116, see Chem. Abstr. 31 (1937) 1950.

234. Hasegawa, H.: Tobacco rutin and the relation of color to quality in dried tobacco leaves; J. Agr. Chem. Soc. Japan 7 (1931) 1036-1049.

235. Kobel, M. and C. Neuberg: Über das Vorkommen von Saponin im Tabak und einer Begleitsubstanz [Presence of saponin in tobacco and an accompanying substance]; J. Prakt. Chem. 143 (1935) 29-41, see Chem. Abstr. 29 (1935) 7586.

236. Neuberg, C. and M. Kobel: Die Umwandlung von Rutin in braune Pigmente durch Tabakenzyme [Conversion of rutin into brown pigment by tobacco enzymes]; Naturwissenschaften 23 (1935) 800-801.

237. Neuberg, C. and M. Kobel: Die Umwandlung von Rutin in braune Pigmente durch tabakeigene Enzyme und die Wirkung dieser Enzyme auf andere phenolische Substanzen [Conversion of rutin into a brown pigment by tobacco enzymes and the effect of these enzymes on other phenolic substances]; Enzymologia 1 (1936) 177-182.

238. Couch, J.F. and C.F. Krewson: Rutin; USDA Agr. Bur. Agr. Ind. Chem. AIC-52 (1944).

239. Couch, J.F. and C.F. Krewson: Rutin - medicinal agent found in bright tobacco; Chem. Digest 3 (1944) 230-231.

240. Griffith, J.Q. Jr, J.F. Couch, and M.A. Lindauer: Effect of rutin on increased capillary fragility in man; Proc. Soc. Exp. Biol. Med. 55 (1944) 228-229.

241. Couch, J.F.: Rutin - a new drug; $1^{\text {st }}$ Tobacco Chemists' Research Conference, Program Booklet and Abstracts, Vol. 1, Paper No. 7, 1947.

242. Badgett, C.O., E.G. Beinhart, J. Maher, and J.A. Connelly: Rutin content of several varieties of Nicotiana rustica and N. glauca; Arch. Biochem. 24 (1949) 245-250.

243. Nio, S. and E. Wada: Chemical constituents of tobacco. I. The isolation of rutin from tobacco flowers; J. Agr. Chem. Soc. Japan 24 (1951) 485-486.

244. Wada, E.: Chemical constituents of tobacco. III. Kaempferol-3-rhamnoglucoside from the flowers of Nicotiana sylvestris; J. Agr. Chem. Soc. Japan 26 (1952) 159-162.

245. Roffo, A.E.: Espectrograffe de los derivados obtenidos por destilacion directa de los tabacos y su relacion como agentes carcinogenos [Spectrograph of derivatives obtained by direct distillation of tobaccos and the relation to carcinogenic agents]; Bol. Inst. Med. Exptl. Estud. Cáncer 14 (1937) 311-399, see Chem. Abstr. 32 (1938) 3812 3 .

246. Roffo, A.H.: 1,2-Benzopirene: Cancerigeno extraido del alquitran del tabaco [1,2-Benzpyrene: A carcinogen extracted from tobacco tar]; Bol. Inst. Med. Exp. Estud. Cáncer 16 (1939) 1-38.

247. Roffo, A.H.: Über die Prinzipien der krebserzeugenden Wirkung des Tabaks [The principles of cancer production by tobacco]; Schweiz. Med. Wchnschr. 71 (1941) 549-552, see Chem. Abstr. 36 (1942) $143^{4}$.

248. Roffo, A.H.: El alquitran de tabaco extraido y la disminucion de cancerizacion [The tar from extracted tobacco and the diminution of carcinogenesis]; Bol. Inst. Med. Exptl. Estud. Cáncer 19 (1942) 431-502, see Chem. Abstr. 37 (1943) 6335.

249. Späth, E. and S. Biniecki: Tobacco alkaloids. XVI. Nmethylpyrrolidine, a new tobacco alkaloid and the constitution of isonicoteine; Ber. Dtsch. Chem. Ges. 72B (1939) 1809-1815, see Chem. Abstr. 33 (1939) 9307.

250. Frankenburg, W.G.: Chemical changes in the harvested tobacco leaf, in Advances in Enzymology, Vol. VI, Interscience, New York, NY (1946) p. 344.

251. Späth, E. and F. Kesztler: Über neue Tabakalkaloide. XI. L-Anatabin, ein neues Tabakalkaloid [On new 
tobacco alkaloids. XI. $L$-Anatabine, a new tobacco alkaloid]; Ber. Dtsch. Chem. Ges. 70 (1937) 239-243.

252. Späth, E. and F. Kesztler: Über neue Tabakalkaloide. XII. Über das Vorkommen von $d, l$-Nornikotin, $d, l$ Anatabin, und $l$-Anabasin im Tabak [On new tobacco alkaloids. XII. On the occurrence of $d, l$-nornicotine, $d, l$-anatabine, and $l$-anabasine in tobacco]; Ber. Dtsch. Chem. Ges. 70 (1937) 704-709.

253. Shmuk, A.A.: New methods of determining nicotine and anabasine content; Collected Works on the Chemistry of Tobacco and Makhorka, VITIM, 14 (1948) 145.

254. Späth, E. and F. Kesztler: Über neue Basen des Tabaks. (XIII. Mitteilung über Tabakalkaloide) [Tobacco alkaloids. XIII. New tobacco bases. Also remarks on our knowledge of rhoeadin, of $l$-peganin, and of ammoresinol]; Ber. Dtsch. Chem. Ges. 70B (1937) 2450-2454, see Chem. Abstr. 32 (1938) 1275.

255. Warrington, K.: The effect of boric acid and borax on the broad bean and certain other plants; Ann. Bol. 37 (1923) 629-672.

256. Sommer, A.L. and C.B. Lipman: Evidence of the indispensable nature of boron and zinc for higher green plants; Plant Physiol. 1 (1926) 231-249.

257. Swanback, T.R.: The effect of boric acid on the growth of tobacco plants in nutrient solutions; Plant Physiol. 2 (1927) 475-486.

258. McMurtrey, J.E.: The effect of boron deficiency on the growth of tobacco plants in aerated and unaerated solutions; Jour. Agr. Res. 38 (1929) 371-380.

259. Van Schreven, D.A.: External and internal symptoms of boron deficiency in tobacco; Tidjdschr. Plantenxiokten 40 (1934) 98-112.

260. Steinberg, R.A.: Nitrogen compounds: Effect of amino acids on the growth of tobacco; $4^{\text {th }}$ Tobacco Chemists' Research Conference, Program Booklet and Abstracts, Vol. 4, Paper No. 1, 1950.

261.Lashkevvich, G.I.: The use of copper- and boroncontaining fertilizers in agricultural plantings on peaty soil; Mikroelementy v. Zhizni Rastenni I. Zhivotnykh. Akad. Nauk S.S.S.R., Trudy Kof. Mikroelemt. 1950 (1952) 302-323.

262. Ciamician, G. and C. Ravenna: Genesis of alkaloids in plants; Atti Accad. Lincei 20 (I) (1911) 614-624, see Chem. Abstr. 5 (1911) 3466.

263. Shmuk, A.A.: Inositol in tobacco; U.S.S.R. State Inst. Tobacco Invest. Bull. 69 (1930) 15-18, see Chem. Abstr. 25 (1930) 3124.

264. Vauquelin, M.: Analyse du tabac à larges feuilles, Nicotiana tabacum, latifolia, et angustifolia [Analysis of tobacco with large leaves, Nicotiana tabacum, latifolia, and angustifolia]; Ann. de Chimie, T 71 (1809) 139.

265.Kissling, R.: Tabakanalyse [Analysis of tobacco]; Chem. Ztg. 28 (1904)775-776.

266. Pucher, G.W., A.J. Wakeham, and H.B. Vickery: The metabolism of the organic acids of the tobacco leaf during culture; J. Biol. Chem. 119 (1937) 523-534.

267. Bacon, C.W., R. Wenger, and J.F. Bullock: Chemical changes in tobacco associated with the flue-curing process; $5^{\text {th }}$ Tobacco Chemists' Research Conference, Program Booklet and Abstracts, Vol. 5, Paper No. 2, 1951.

268. Bacon, C.W., R. Wenger, and J.F. Bullock: Chemical changes in tobacco during flue-curing; Ind. Eng.
Chem. 44 (1952) 292-296.

269. Phillips, M. and A.M. Bacot: The chemical composition of certain grades of Type II American flue-cured tobacco: Relationship of composition to grade characteristics; J. Assoc. Off. Agr. Chem. 36 (1953) 504-524.

270. Wright, H.E. Jr and H.H. Burton: Effect of priming on the chemical changes that occur during the curing of burley tobacco; $7^{\text {th }}$ Tobacco Chemists' Research Conference, Program Booklet and Abstracts, Vol. 7, Paper No. 7, 1953, p. 2.

271.Neuberg, C. and M. Kobel: Über Bildung von Diketonen aus Bestandteilen des Tabaks [On the formation of diketones from tobacco components]; Biochem. Z. 275 (1935) 339-343, see Chem. Abstr. 29 (1935) 2511.

272. Schmalfuss, H.: Diacetyl - Ein Aromabestandteil des Tabakrauches [Diacetyl: A constituent contributing to the aroma of tobacco smoke] ; Tabak (Berlin) 3 (1939) 19-21.

273. Schmalfuss, H.: Diacetyl: An aroma component of tobacco smoke; Rev. Internat. Tabaks 25 (1950) 89, see Chem. Abstr. 45 (1951) 1303e.

274. Sasaki, T.: The chemical components of tobacco smoke. Diacetyl content of cigarette smoke; J. Agr. Chem. Soc. Japan 27 (1953) 134-136.

275. Schmalfuss, H. and H. Barthmeyer: Diacetyl - ein Aromastoff in Lebensmitteln und Gewürzen [Diacetyl as an aromatic component of foodstuffs and condiments]; Biochem. Z. 216 (1929) 330-335.

276. Schmalfuss, H. and H.B. Schmalfuss: Die Bestimmung von Diacetyl und Azetylmenthylcarbinol in Lebensmitteln [Detection of diacetyl and acetylmenthylcarbinol in foodstuffs]; Z. Untersuch. Lebensm. 63 (1932) 283-288, see Chem. Abstr. 22 (1928) 3186; 24 (1930) 1432; 27 (1933) 536.

277. Thoms, H.: Versuche zur Entgiftung des Tabakrauches [Study of the detoxification of tobacco smoke]; Chem. Ztg. 28 (1904) 1-3.

278. Wenusch, A.: Beitrag zur Kenntnis des Tabakrauchs [Contribution to the knowledge of tobacco smoke]; Pharm. Zentralhalle 76 (1935) 297-300.

279. Wenusch, A.: Untersuchungen über die Nikotinbildung in der Tabakpflanze [Investigation of nicotine formation in the tobacco plant]; $Z$. Lebensm. Untersuch. 88 (1948) 629-633, see Chem. Abstr. 43 (1949) 4345.

280. Garner, W.W.: The relation of the composition of the leaf to the burning qualities of tobacco; U.S. Dept. Agr., Bureau Of Plant Industry, Bulletin 105 (1907).

281. Bailey, C.W. and P.J. Anderson: Chemical analyses of tobacco from the nitrogen plots; Conn. Agr. Expt. Sta., Tobacco Sta. Bull. 10 (1928) 35T-50T.

282. Bortner, C.E. and J.L. Hamilton: Nitrogen and mineral uptake of burley tobacco during the 1952 season; $7^{\text {th }}$ Tobacco Chemists' Research Conference, Program Booklet and Abstracts, Vol. 7, Paper No. 11, 1953, pp.6-7.

283. Garner, W.W., C. W. Bacon, and J.D. Bowling Jr: Cigarette and cigar tobaccos; Ind. Eng. Chem. 26 (1934) 970-974.

284. Garner, W.W., C.W. Bacon, J.D. Bowling Jr, and D. E. Brown: The nitrogen nutrition of tobacco; U.S. Dept. Agr. Tech. Bull. 414 (1934). 
285. Habermann, J.: Beitrag zur Kenntnis des Zigarrenrauches [Contribution to the knowledge of cigar smoke]; Hoppe-Seyler's Z. Physiol. Chem. 33 (1901) 55-125.

286. Moir, J.: The composition of tobacco smoke; Proc. Chem. Metallurg. Mining Soc. (South Africa) 4 (1903/1904) 525-532

287. Habermann, J.: Beitrag zur Kenntnis des Zigarren- und Pfeifenrauches [Contribution to the knowledge of cigar and pipe smoke]; Hoppe-Seyler's Z. Physiol. Chem. 40 (1904) 148-161.

288. Bush, A.D.: Tobacco smoking and mental efficiency; New York Med. J. 99 (1914) 519-527.

289. Armstrong, H.E. and E.V. Evans: Carbonic oxide in tobacco smoke; Brit. Med. J. (1922) (i) 922-923.

290. Saruta, N.: Über die quantitative Bestimmung der Kohlenoxydmenge im Tabakrauch [On the quantitative determination of the amount of carbon monoxide in tobacco smoke]; Fukuoka Acta Med. 31 (1938) 180.

291. Wenusch, A. and R. Schöller: Zusammensetzung und Charakterisierung des gasförmigen Anteils (der dispergierenden Phase) des Tabakrauchs [Composition and characteristics of the gaseous part (the dispersed phase) of tobacco smoke]; Z. Untersuch. Lebensm. 75 (1938) 346-353.

292.De Voogd, J.G. and A. Van der Linden: Carbon monoxide in tobacco smoke; Het. Gas 59 (1939) $165-166$.

293. de Campos, M.D.: Tobacco and cigar smoke; Anais Faculdade Farm. Odontol. Unic. (Sao Paulo) 1 (1939/1940) 15-24, see Chem. Abstr. 39 (1945) 5395.

294. Fishel, J.B. and J.F. Haskins: Composition of cigarette smoke. The gaseous phase; Ind. Eng. Chem. 41 (1949) 1374-1376.

295. Boekhout, F.W.J. and O. de Vries: Fermentation of tobacco; Cent. Bakt. Parasitenk. II Abt. 24 (1909) 495-511, see Chem. Abstr. 4 (1910) 336.

296. Krause, O.: Kohlenmonoxid im Tabakrauch [Carbon monoxide in tobacco smoke]; Dingler's Polyech. J. 213 (1874) 495-497.

297. Schwarz, H.: Die Analyse des Rauches von Virginia Zigarren [Analysis of the smoke of Virginia cigars]; Dingler's Polyech. J. 226 (1877) 305-307.

298. Fokker, A.P.: The hygienic effect of tobacco smoke; Nederland. Tijdschr. Geneeskunde 20 (1884) 737-740.

299. Wahl, F.: Kohlenmonoxid im Tabakrauch [Carbon monoxide in tobacco smoke]; Arch. Gesammte Physiol. Mensch. Tiere 28 (1899) 262-285.

300. Thoms, H.: Kohlenmonoxid im Tabakrauch [Carbon monoxide in tobacco smoke]; Ber. Deut. Pharm. Gesellschaft. 10 (1900) 19.

301. Spitta: Die Bestimmung kleiner Mengen von Kohlenmonoxid in der Luft [The determination of small amounts of carbon monoxide in the air]; Arch. Hyg. 45 (1903) 285-310.

302. Marcelet, H.: On the determination of carbon monoxide, particularly in tobacco smoke; Pharmacy Thesis No. 109, Montpelier University (1907).

303. Tani, T.: Untersuchungen über die Bestimmung von Kohlenmonoxid im Tabakrauch und seine Bedeutung für die Gesundheit [Investigations on the determination of carbon monoxide in tobacco smoke and its health significance]; Thesis, Würzburg University (1907) pp. 1-25.
304. Toth, J,: Contribution to the determination of carbon monoxide in tobacco smoke; Chem. Ztg. 31 (1907) 98-99.

305. Fleig, C.: Does carbon monoxide contribute to the toxicity of cigarette smoke? Compt. Rend. Acad. Sci. 146 (1908) 776-779.

306. Marcelet, H.: On the determination of carbon monoxide, particularly in tobacco smoke; Bull. Soc. Chim. France 3-4 (1908) 556-558.

307. Toth, J,: Contribution to the determination of carbon monoxide in tobacco smoke; Rev. Internat. Falsifications 21 (1908) 8-11.

308. Marcelet, H.: On the determination of carbon monoxide, particularly in tobacco smoke; Z. Anal. Chem. 50 (1911) 315-316.

309. Hartridge, H.: Carbon monoxide in tobacco smoke; J. Physiol., Proc. Physiol. Soc. Mtg. 53 (1920) 82-83 [lxxxii-lxxxiii], see Chem. Abstr. 14 (1920) 5281.

310. Baumberger, J.P.: The carbon monoxide content of tobacco smoke and its absorption on inhalation; J. Pharm. Exp. Therap. 21 (1923) 23-34.

311.Heinz, R.: Über die Giftigkeit des Tabakrauches, inbesondere Zigarettenrauches [On the toxicity of tobacco smoke, especially cigarette smoke]; Deut. Med. Wchnschr. 49 (1923) 318-319.

312.Jones, G.W., Y. Yant, and L.B. Berger: Carbon monoxide hazards from tobacco smoke; U.S. Bureau of Mines, Repts. of Investigat., Rpt. No. 2539 (1923) pp. 1-6.

313. Schöller, R.: Der Gehalt des Tabakrauches an Kohlenoxid [The carbon monoxide content of tobacco smoke]; Fachl. Mitt. Österr. Tabakregie 1929(3) 1-9.

314. Ehrismann, O. and G. Abel: The carbon monoxide content of cigarette smoke; Z. Hyg. Infektionskrankh. 116 (1934) 4-10

315. Leikola, E. and E. Rautavaara: Der Anteil des Kohlenmonoxids an der durch das Tabakrauchen verursachten Blutdrucksteigerung [The importance of carbon monoxide in the increase in blood pressure caused by tobacco smoking]; Acta Soc. Med. Finn. Duodecim. 16 (1934) 3-25.

316. Waser, E. and M. Stähli: Untersuchung des Tabakrauches. IV [Investigation of tobacco smoke. IV]; Z. Untersuch. Lebensm. 67 (1934) 280-284.

317. Wenusch, A. and R. Schöller: Dem Kohlenoxyd des Tabakrauches kommt keine blutdrucksteigernde Wirkung zu [Carbon monoxide from tobacco smoke causes no rise in blood pressure]; Med. Klin. 31 (1935) 554.

318. Saruta, N.: Über die quantitative Bestimmung der Kohlenoxydmenge im Tabakrauch [On the quantitative determination of the amount of carbon monoxide in tobacco smoke]; Fukuoka Acta Med. 30 (1937) $117-118$.

319. Tsumura, H.: Carbon monoxide content of tobacco smoke; J. Chosen Med. Assoc 27 (1937) 926-929, see Chem. Abstr. 32 (1938) 1867.

320. Roffo, A.E.: El óxido de carbono en la sangre de los fumadoress [Carbon monoxide in the blood of smokers]; Bol. Inst. Med. Exptl. Estud. Cáncer 14 (1938) 689-709, 17 (1940) 282.

321. Hukusima, Y.: Tobacco constituents. III. Carbon monoxide in tobacco smoke. I. Method of its measurement; J. Chem. Soc. (Japan) 61 (1942) 889-903, see Chem. 
Abstr. 36 (1942) 7240.

322. Copenhaver, J.W. and M.H. Bigelow: Acetylene and carbon monoxide chemistry; Reinhold, New York, NY (1949) p. 127.

323. Obersteg, J.I. and M. Scoch-Kanter: Die Kohlenoxydaufnahme bei Rauchern und Nichtrauchern [Carbon monoxide uptake by smokers and nonsmokers]; Gesundheit 31 (1951) 89-99.

324. Garner, W.W., E.G. Moss, H.S. Yohe, F.B. Wilkinson, and O.C. Stine: History and status of tobacco culture; U.S. Dept. Agr. Yearbook, 1922 (1923) 395-468.

325. Garner, W.W., J.E. McMurtrey, C.W. Bacon, and E.G. Moss: Sand drown, a chlorosis of tobacco due to magnesium deficiency and the relation of sulfates and chlorides of potassium to the disease; J. Agr. Res. 23 (1923) 27-40.

326. Nagel, W.: Über die Blattfarbstoffe des Tabaks [Leaf pigments of tobacco]; Botan. Arch. 40 (1939) 1-57, see Chem. Abstr. 34 (1940) 2883.

327. Jeffrey, R.N. and R.B. Griffith: Changes in the chlorophyll and carotene contents of curing burley tobacco cut at different stages of maturity; Plant Physiol. 22 (1947) 34-41.

328. Hukusima, Y.: Tobacco constituents. The enzyme content of flue-cured tobacco after redrying; J. Chem. Soc. (Japan) 59 (1938) 1079.

329. Brown, J.C. and R.A. Steinberg: Iron and copper enzymes in leaf lamina of tobacco when deficient in micro-nutrients or grown on calcareous and organic soils; Plant Physiol. 28 (1953) 488-494.

330. Tracey, M.V.: Cellulase from leaves and roots of tobacco; Biochem. J. 47 (1950) 431-433.

331. Pyriki, C: Die Kohlehydrate des Tabaks und ihre Bedeutung [The carbohydrates of tobacco and their significance]; Z. Untersuch. Lebensm. 68 (1934) 554-556.

332. Brückner, H.: Die Biochemie des Tabaks [The biochemistry of tobaccos]; P. Parey, Berlin (1936).

333. Shabanov, I.M.: The phosphatides in Nicotiana rustica seeds; Vsesoyuz. Nauch. Issledovatil Inst. Tabach. Makhoroch Prom. 140 (1939) 92-96, see Chem. Abstr. 34 1940) 4862.

334. Yamagata, N.: Trace elements in Japanese tobacco; J. Chem. Soc. Japan, Pure Chem Sect. 71 (1950) 288.

335. Grandeau, L.: Compt. Rend. Acad. Sci. Paris 54 (1862) 1057-1059, see Grandeau, L.: Recherches sur la présence du rubidium et du caesium dans les eaux naturelles, les minéraux, et les végétaux [Research on the presence of rubidium and cesium in natural water, minerals and plants]; Paris, Mallet-Bachelier 1 (1863) 84, Ann. Chim. Phys. $3^{\text {rd }}$ Series (1863) lxvii.

336. Nessler, J.: Über den Anbau und die Behandlung des Tabaks [The culture and treatment of tobacco]; Landw. Vers. Stat. 40 (1892) 395-438.

337.Pitchard, P.: Contributions to the study of the forms and conditions under which the chlorine of the soil ordinarily enters plants; Compt. Rend. Acad. Sci. Paris 128 (1899) 615-617.

338. Krevs, K.J.: Chlorine in tobacco; State Inst. Tobacco Invest. Bull. 23 (1925) 3.

339. Ward, G.M.: Mineral-absorption studies with tobacco; The Lighter 11(1) (1941) 16-22.

340. McEvoy, E.T.: The effect of chlorine on tobacco; Lighter 20(3) (1950) 14-15; Soils Fert. XIII (1950) 74.

341. Mosley, J.M., W.R. Harlan, and H.R. Hanmer: Burley tobacco. Relation of the nitrogenous fraction to smoking quality; Ind. Eng. Chem. 43 (1951) 2374.

342. Wenusch, A.: Die Harze des Zigarrenrauches [The resins of cigar smoke]; Z. Untersuch. Lebensm. 73 (1937) 189-191.

343. Kennaway, E.L. and B. Sampson: Tumours of skin and mammary gland caused by pyrogenous products of cholesterol; J. Path. Bacteriol. 31 (1928) 609-612.

344.Roffo, A.H.: Die Bildung von Geschwüren und bösartigem Wachstum im Darmtrakt nach der Verabreichung von Essen mit bestrahltem Cholesterin [The formation of ulcers and malignant growths in the alimentary tract after administration of food with irradiated cholesterol]; Z. Krebsforsch. 47 (1938) 473-497.

345. Roffo, A.H.: La acción de cancerígeno de un derivado phenanthrene del colesterol [Cancer-producing action of a phenanthrene derivative from cholesterol]; Bol. Inst. Med. Exptl. Estud. Cáncer 15 (1939) 837-845, see Chem. Abstr. 33 (1939) 82777.

346. Roffo, A.H.: Die karzinogene Wirkung eines Phenanthrenderivates aus Cholesterin [The carcinogenic action of a phenanthrene derivative from cholesterol]; Z. Krebsforsch. 49 (1939) 341-347, see Chem. Abstr. 34 (1940) $1730^{8}$.

347. Roffo, A.H.: Pirolisis de colestrol. Alquitrán cancerígene de cholesterol [Pyrolysis of cholesterol. Carcinogenic tar from cholesterol]; Bol. Inst. Med. Exptl. Estud. Cáncer 18 (1941) 929-943, see Chem. Abstr. 36 (1942) 62219.

348. Kirby, A.H.M.: Attempts to induce stomach tumors. I. Effect of cholesterol heated to $300{ }^{\circ} \mathrm{C}$; Cancer Res. 3 (1943) 519-525.

349. Falk, H.L., S. Goldfein, and P. Steiner: The products of cholesterol at $360{ }^{\circ} \mathrm{C}$ and their relation to carcinogens; Cancer Res. 9 (1949) 438-447.

350. Johnson, C.E.: A study of cigar tobacco with reference to the less abundant elements; Thesis. Columbia University, New York, NY (1936).

351. Nicholas, D.J.D.: Some effects of metals in excess on crop plants grown in solution culture. III. Effects of cobalt, nickel, and zinc on growth, metal, and chlorophyll contents of tomato; Ann. Rept. Agr. Hort. Res. Sta., Long Ashton, Bristol (1952) 87-102.

352. Nicholas, D.J.D. and W.D.E. Thomas: Some effects of metals in excess on crop plants grown in solution culture. I. Effects of cobalt on fertilizer and soil phosphate uptake; Plant Soil 5 (1953) 67-80.

353. Manns, T.F., W.L. Churchman, and M.M. Manns: Influence of the minor elements on the soil flora; Del. Agr. Expt. Sta. Bull. 207 (1937) 45-46.

354. Swanback, T.R.: Copper ion in tobacco production; Conn. Agr. Expt. Bull. 535 (1950) 3-11.

355. Gorter, K.: Die Verbreitung von Chlorogensäure in der Natur [Distribution of chlorogenic acid in nature]; Arch. Pharm. 217 (1909) 184-196.

356. König, R. and W. Dörr: Über die Chemie des Tabaks. I [On tobacco chemistry. I]; Biochem. Ztschr. 263 (1933) 295-301.

357. Shmuk, A.A.: Investigations of the tobacco acids (Second Report); U.S.S.R. State Inst. Tobacco Invest. Bull. 69 (1930) 19.

358. Shmuk, A.A.: The presence of inosite in tobacco; State Inst. for Tobacco Invest., Krasnodar, Bull. 69 (1930) $7-14$. 
359. Schumacher, J.N. and L.L. Vestal: Turkish tobacco. Isolation and identification of some components of the essential oil of Turkish tobacco dust; RDR, 1962, No. 18, June 25, see www.rjrtdocs.com 500938952 -8981; Isolation and identification of some components of Turkish tobacco; 27th Tobacco Chemists' Research Conference, Program Booklet and Abstracts, Vol. 27, Paper No. 47, 1973, p. 31; Tob. Sci. 18 (1974) 43-48; Miller, C.W., D.L. Roberts, R.A. Lloyd Jr, J.P. Dickerson, C.E. Rix, P.H. Ayers, and N.H. Nelson: Composition of flue-cured tobacco; RDR, 1973, No. 10, October 25, see www.rjitdocs.com 501003209 3306; Lloyd, R.A. Jr, C.W Miller, D.L. Roberts, J.A. Giles, J.P. Dickerson, N.H. Nelson, C.E. Rix, and P.H. Ayers: Flue-cured tobacco flavor. I. Essence and essential oil components; CORESTA 1974 Symp., Montreux, Switzerland, 1974; Tob. Sci. 20 (1976) 40-48; Leffingwell, J.C. and E.D. Alford: Volatile constituents of Perique tobacco; Electronic J. Environ. Agr. Food Chem. 4 (2005) 899-915.

360. Enzell, C.R. and I. Wahlberg: Leaf composition in relation to smoking quality and aroma; Recent Adv. Tob. Sci. 6 (1980) 64-122; Wahlberg, I. and C.R. Enzell: Tobacco isoprenoids [A review of literature between 1975 and 1984]; Natural Products Rept. 4(3) (1987) 237-276.

361. Haggard, H.W. and L.A. Greenberg: Concentration of menthol in the smoke from mentholated cigarettes. A study of local and systemic effects; Arch. Otolaryngol. 33 (1941) 711-716.

362. Rakieten, N., M.L. Rakieten, D. Feldman, and M.J. Boykin Jr: Mammalian ciliated respiratory epithelium: Studies with particular reference to effects of menthol, nicotine, and smoke of mentholated cigarettes; Arch. Oto-Laryngol. 56 (1952) 494-503.

363. Sharmon, C.F.: Imperial Tobacco Co., Tobacco Symposium, Am. Chem. Soc. Convention, New York, NY (1951).

364. Silberger, J. and F. Skoog: Changes induced by indoleacetic acid in nucleic acid contents and growth of tobacco pith tissue; Science 118 (1953) 443-444.

365. Unterdorden, O.: On the behavior of organic substances at high temperatures. II. Distillates of plant materials; Poggendorff's Ann. Physik 8 (1826) 397-410.

366. Holden, M.: A study of enzymes that can break down tobacco-leaf components. 3. Fungal polygalacturonase on leaf fibre; Biochem J. 47 (1950) 415-420.

367. Nottbohm, F.E. and F. Mayer: Über das Vorkommen und die Bestimmung von Cholin in Samen und Blättern des Tabaks [Occurrence and detection of choline in tobacco seeds and leaves]; Z. Untersuch. Lebensm. 63 (1932) 620-623, see Chem. Abstr. 27 (1933) 1986.

368. Toth, J.: Der Gehalt an Cyanverbindungen des Tabakrauchs [The content of cyanogen compounds in tobacco smoke]; Chem. Ztg. 34 (1910) 298-299.

369. Toth, J.: Die Cyanverbindungen des Tabakrauchs [The cyanogen compounds of tobacco smoke]; Chem. Ztg. 34 (1910) 1357.

370.Toth, J.: Der Gehalt an Cyanverbindungen des Tabakrauchs [The content of cyanogen compounds in tobacco smoke]; Chem. Ztg. 35 (1911) 1262.

371. Wiley, F.H., W.C. Hueper, D.S. Bergen, and F.R. Blood: The formation of oxalic acid from ethylene glycol and related solvents; J. Ind. Hyg. Toxicol. 20 (1938) 269-277.

372.Forbes, J.C. and H.B. Haag: Hygroscopic agents in cigaret smoke; Ind. Eng. Chem. 30 (1938) 717-718.

373. Reif, G.: Die Bestimmung von Äthylenglykol im Tabakrauch mittels 2-Naphthol [The determination of ethylene glycol in tobacco smoke using 2-naphthol]; Pharmazie 4 (1949) 110-113.

374. McNally, W.D., W. Bergman, and R.H.K. Foster: Irritative effects of smoke from tobacco treated with glycerine and diethylene glycol; Illinois Med. J. 87 (1945) 250-252.

375.Haag, H.B.: Studies on the physiologic action of diethylene glycol. I. The effect upon the irritating action and toxic properties of cigarette smoke; J. Lab. Clin. Med. 22 (1937) 341-346.

376. Holck, H.G.O. and A.J. Carlson: Alleged influence of glycerine and diethylene glycol upon the irritating qualities of cigarette smoke; Proc. Soc. Exp. Biol. 36 (1937) 302-307.

377. Newman, B.A.: Dermatitis caused by diethylene glycol in tobacco; J. Am. Med. Assoc. 111 (1938) 25.

378. Küchle, H.-J., A. Loeser, G. Meyer, and E. Stürmer: Untersuchungen über Diäthylenglykol als Tabakfeuchthaltemittel [Research on diethylene glycol as a tobacco humectant]; Z. Ges. Exp. Med. 119 (1952) 266-271.

379. Kissling, R.: Fett des Tabaks [Tobacco fat]; Ber. Dtsch. Chem. Ges. 16 (1883) 2432-2434.

380. Hirst, E.L.: The role of tobacco smoking in the production of cancer. IV. Report on the spectrographic examination of tobacco tar; J. Hyg. 32 (1932) 300.

381. Mizukami, Y.: Chemical constituents of tobacco plants. I. A fluorescent substance in tobacco plant; J. Agr. Chem. Soc. Japan 25 (1951/1952) 531-533.

382. Spira, L.: Fluorine in cigarette smoke; Acta Med. Scand. 130 (1948) 78

383. Trillat, A.: Formaldehyd im Tabakrauch [Formaldehyde in tobacco smoke]; Chem. Ztg. 28 (1904) 1149 .

384. Trillat, A.: On the formation of formaldehyde during the combustion of tobacco; Compt. Rend. 139 (1904) 724-744.

385. Trillat, A.: Contribution à l'étude sur la fumée du tabac [Contribution to the study of tobacco smoke]; Compt. Rend. Soc. Biol. 33 (1904) 469.

386. Trillat, A.: On the presence and formation of formaldehyde during combustion; Bull. Soc. Chim. Paris 33 (1905) 386-393.

387. Neuberg, C. and B. Ottenstein: Übertritt von Methylalkohol in den Tabakrauch [Appearance of methanol in tobacco smoke]; Biochem. Z. 188 (1927) 217-226, see Chem. Abstr. 22 (1928) 666.

388. Neuberg, C. and M. Kobel: Isolierung von Methanol aus Tabakrauch [Isolation of methanol from tobacco smoke]; Biochem. Z. 206 (1929) 240-244.

389. Shmuk, A.A. and S. Kashirin: The quantitative content of formic acid in tobacco; U.S.S.R. State Inst. Tobacco Invest. Bull. 69 (1930) 29.

390. Attfield, J.: Über den Zucker der Tabakblätter [On the sugar in tobacco leaves]; Pharm. J. Trans. 14 (1884) 541-542.

391. Shmuk, A.A.: An investigation of the carbohydrates of tobacco; Collected Works on the Chemistry of Tobacco, V (1934) p. 3. 
392. Schürch, O. and A. Winterstein: Experimentelle Untersuchungen zur Frage Tabak und Krebs [Experimental investigation of the question of tobacco and cancer]; Z. Krebsforsch. 42 (1935) 76-92.

393. Thoms, H.: Deutsches Opium [German opium]; Apoth. Ztg. 19 (1904) 773-774.

394. Molinari, E.: Über Aldehyde und Ketone des Tabakrauches [The aldehydes and ketones in tobacco smoke]; Fachl.. Mitt. Österr. Tabakregie 1933(2) $23-25$.

395. Jetta, G.: Artificial drying of tobacco; Boll. Tecn. Della Collt. dei Tabacci 2 (1903) 299; 3 (1904) 25; 5 (1906) 42.

396. Neuberg, C. and M. Kobel: Fortgesetzte vergleichende Versuche über die Vergärbarkeit freier und phosphorylierter Hexosen und über eine polarimetrisch feststellbare Bindung dieser Substanzen an Inhaltsstoffen der Hefezelle [Continuation of comparative experiments of fermentability of free and phosphorylated hexoses and on a polarimetric determinable binding of these subtances to the components contained in yeast cells]; Biochem. Z. 179 (1926) 451.

397. Hukusima, Y, and K. Ooike: Tobacco constituents. IV. Essential oil and resin of tobacco; J. Chem. Soc. (Japan) 61 (1940) 1297-1301.

398. Neuberg, C. and M. Scheuer: Pektine des Tabaks [Pectins of tobacco]; Biochem. Z. 243 (1931) 461-471.

399. Miller, L.P.: High yields of $\beta-2,2,2$-trichloroethyl- $D$ glucoside and $\beta$-2,2,2-trichloroethyl gentiobioside from tobacco plants treated with chloral hydrate; Contrib. Boyce Thompson Inst. 13 (1943) 185-200, see Chem. Abstr. 38 (1944) 956.

400. Vickery, H.B.: Chemical investigations of the tobacco plant. II. The globulin of the tobacco seed; Conn. Agr. Expt. Sta. Bull. 339 (1932).

401. Wenusch, A.: Über das Auftreten von Lävoglukosan im Tabakrauch [The occurrence of levoglucosan in tobacco smoke]; Fachl. Mitt. Österr. Tabakregie 1938(2) 4-5, see Chem. Abstr. 35 (1941) 4157.

402. Wenusch, A.: Reduktionsvermögen des Hauptstromrauches vor and nach der Hydrolyse [Reducing activity of mainstream smoke before and after hydrolysis]; Fachl. Mitt. Österr. Tabakregie 1938(2) 10-11.

403. Vickery, H.B., G.W. Pucher, and H.E. Clark: Glutamine in the tobacco plant; Science 80 (1934) 459-461.

404. Heiserich, E.: Der Metabolismus des Schwefels in Mais und Tabak [Sulfur metabolism in maize and tobacco]; Z. Pflanzennahr. Düngung Bodenk. 37 (1935) 55-72, see Chem. Abstr. 29 (1936) 5151.

405. Yamafuji , K.: Two new glucosides from tobacco leaves; J. Agr. Chem. Soc. 8 (1932) 404-410.

406. Geissmann, T.A. and E. Hinreiner: Theories of the biogenesis of flavonoid compounds; Botan. Rev. 18 (1952) 77-164.

407. Kissling, R.: Beiträge zur Kenntnis des Tabakrauches [Contribution to the knowledge of tobacco smoke]; Arch. Hyg. 20 (1894) 211-213.

408. Wenusch, A.: Über das Vorkommen von höheren Kohlenwasserstoffen im Tabakrauch [Occurrence of higher hydrocarbons in tobacco smoke]; Biochem. Z. 273 (1934) 178-179

409. Wenusch, A.: Wissenschaftliche Grundlagen für die Beurteillung der Stärke von Rauchwaren [Scientific basis for the determination of the strength of smoking materials]; Z. Untersuch. Lebensm. 73 (1937) 176-185.

410. Chibnall, A.C., S.H. Piper, A. Pollard, E.F. Williams, and P. Sahai: The constitution of the primary alcohols, fatty acids and paraffins present in plant and insect waxes; Biochem. J. 28 (1934) 2189-2208.

411. Pyriki, C.: Zur Bestimmung der Harze und Wachse im Tabak [Determination of the resin and wax in tobacco]; Z. Untersuch. Lebensm. 84 (1942) 225-230.

412. Shirokaya, V.N.: Resin of Nicotiana rustica; Sborn Rabot Khim.Tabak Bull. 125 (1935) 151-162, see Chem. Abstr. 30 (1936) 4993.

413. Shmuk, A.A.: On the identification of fatty materials in the composition of tobacco leaves. Collected works on the chemistry of tobacco and Makhorka; Vsesoyuznuii Inst. Tabaknoi Prom. 133 (1937) 3-9, see Chem. Abstr. 32 (1938) 4723.

414. Salisbury, L.F.: The lipids of Connecticut shade-grown tobacco seed; J. Biol. Chem. 117 (1937) 21-25.

415. Hasegawa, H.: On the chemical properties of the flavon-derivatives contained in tobacco leaves with special reference to the colour and qualities of cured leaves; Bull. Agr. Chem. Soc. Japan 7 (1931) 73-74.

416. Neuberg, C. and M. Kobel: Die Isolierung von Sorbit aus Tabak [Isolation of sorbitol from tobacco]; $\mathrm{Z}$. Untersuch. Lebensm. 72 (1936) 116-121.

417. Thorpe, T.E. and J. Holmes: The occurrence of paraffins in the leaf of tobacco; J. Chem. Soc. 79 (1901) 982.

418. Vogel, A. and C. Reischauer: Der Schwefelwasserstoff- und Prussinsäuregehalt des Tabakrauches [The hydrogen sulfide and prussic acid content of tobacco smoke]; Dingler's Polytech. J. 148 (1858) 231-233.

419. Poggiale, $M$. and $H$. Marty: Examination of hydrocyanic acid in tobacco smoke; J. Pharm. Chim. 11 (1870) 216-218.

420. Molnár, I.: Chemical examination of tobacco smoke; Budapest (1882).

421. Habermann, J.: Der Blausäuregehalt des Zigarrenrauches [The hydrocyanic acid content of cigar smoke]; Hoppe-Seyler's Z. Physiol. Chem. 37 (1903) $1-17$.

422. Thoms, H.: Der Blausäuregehalt des Zigarrenrauches [The hydrogen cyanide content of cigar smoke]; Hoppe-Seyler's Z. Physiol. Chem. 37 (1903) 250.

423. Habermann, J. and R. Ehrenfeld: Beitrag zur Kenntnis des Zigarrenrauches [Contribution to the knowledge of cigar smoke]; Hoppe-Seyler's Z. Physiol. Chem. 56 (1909) 363-372.

424.Lehmann, K.B. and K. Gundermann: Neue Untersuchungen über die Bedeutung der Blausäure für die Giftigkeit des Tabakrauches [New investigations on the significance of hydrocyanic acid in the toxicity of tobacco smoke]; Arch. Hyg. 76 (1912) 98-115.

425. Schöller, R.: Über den Gehalt des gasförmigen und des fest-flüssigen Anteils des Tabakrauches an Cyanwasserstoff [On the content of hydrogen cyanide in the gas and particulate phases of tobacco smoke]; Fachl. Mitt. Österr. Tabakregie 1938(1) 7-10.

426. Schöller, R.: Schwefelwasserstoff und Rhodanwasserstoff in den gasförmigen und fest-flüssigen Anteilen des Tabakrauches [Hydrogen sulfide and thiocyanic acid in the gas and particulate phases of tobacco smoke]; Fachl. Mitt. Österr. Tabakregie 
1938(2) 1-4.

427. Haag, H.B. and P.S. Larson: Some basic facts of interest concerning cigarettes and their smoke; Interne 14 (1948) 110-116, 119.

428. Toth, J.: Schwefelwasserstoff im Rauch ungarischer Tabake [Hydrogen sulfide in the smoke of Hungarian tobacco]; Chem. Ztg. 37 (1913) 897-898.

429. Wenusch, A.: Über das Auftreten von Schwefelwasserstoff im Tabakrauch [The occurrence of hydrogen sulfide in tobacco smoke]; Z. Untersuch. Lebensm. 70 (1935) 201-204, see Chem. Abstr. 29 (1935) 8228.

430. Ballenger, H.C. and V.H. Johnson: Effects on the throat and conjunctiva of the hygroscopic agents used in cigarets; Arch. Otolaryn. 25 (1937) 75-80.

431. Ballenger, H.C.: Irritation of the throat from cigaret smoke. A study of hygroscopic agents; Tr. Am. Larng. Assoc. 60 (1938) 289-298.

432. Ballenger, H.C.: Irritation of the throat from cigaret smoke. A study of hygroscopic agents; Arch. Otolarg. 29 (1939) 115-123.

433. Mulinos, M.G. and R.L. Osborne: Pharmacology of inflammation. III. Influence of hygroscopic agents on irritation from cigarette smoke; Proc. Soc. Exp. Biol. 32 (1934) 241-245.

434. Greenwald, W.F.: Influence of hygroscopic agents on irritation from cigarette smoke; Med. Record 142 (1935) 496-497.

435. Mulinos, M.G. and R.L. Osborne: Irritating properties of cigarette smoke as influenced by hygroscopic agents; New York State J. 35 (1935) 590-592.

436. Küchle, H.-J., A. Loeser, G. Meyer, C.G. Schmidt, and E. Stürmer: Tabakrauch. Ein Beitrag zur Wirkung von Tabakfeuchthaltemitteln [Tobacco smoke. A contribution to the action from tobacco humectants]; Z. Ges. Exp. Med. 118 (1952) 554-572.

437. Schwaibold, J.: Iodine in tobacco; J. Biochem. 218 (1930) 318.

438. Fesca, M. and E. Imai: Iron in tobacco; Landw. Jahrb. 329, in J. Soc. Chem. Ind. 759 (1888).

439. Coolhaas, C.: Javanese tobacco; Proufata. Vorstenland, Tabak Medel. 68 (1930) 1.

440. Starkenstein, E. and W. Stejskal: Der katalytische Effekt von Eisensalzen auf die Zersetzung von Nikotin während des Rauchens [The catalytic effect of iron salts upon the decomposition of nicotine during smoking]; Arch. Exp. Pathol. Pharm. 172 (1933) 119-136.

441. McMurtrey, J.E.: Cross transfer of mineral nutrients in the tobacco plant; J. Agr. Res. 55 (1937) 475-482.

442. LeCompte, S.B.: Plastid pigments and commercial quality in Connecticut shade tobacco; Am. J. Botany 33 (1946) 726.

443. Noga, E.: Alkaloids of tobacco extract; Fachl. Mitt. Österr. Tabakregie,(1914) 1, see J. Chem. Soc. 108 (1915) 711.

444. Creegan, J.J.: Case of lead poisoning caused by the use of snuff; Med. Times Gaz. (London) 2 (1861) 443.

445. Cadenhead, A.F.G. and A.G. Jacques: A possible source of lead poisoning; Canadian Chem. Met. 5 (1921) 260.

446. Prat, S.: The absorption of lead by plants; Am. J. Botany 14 (1927) 633-634.

447. Reitzel, R.J.: Lead poisoning from snuff; Ann. Int. 3 (1929/1930) 378-385.
448. Shabanov, I.M.: The phosphatides of tobacco seeds; Vsesoyuz. Inst. Tabach. I. Marhoroch Prom. 133 (1937) 78-85, see Chem. Abstr. 32 (1938) 5444.

449. Ravenna, C. and M. Zamorani: The behavior of plants towards lithium salts; Atti. R. Acad. Lincei, Rend. Cl. Sci. Fis. Mat. Nat. 5, Ser. 18, II(12) (1909) 626-630, see Abstr. in J. Chem. Soc. (London) (1910) 98.

450. Garner, W.W., J.E. McMurtrey, and E.G. Moss: Sand drown. A chlorosis of tobacco and other plants resulting from magnesium deficiency; Science 56 (1922) 341-342.

451. Anderson, P.J., T.R. Swanback, and O.E. Street: The relation of magnesia to burning qualities of cigar leaf tobacco; Connecticut State Sta. Bull. 326 (1931) 391-398.

452. Anderson, P.J., O.E. Street, and T.R. Swanback: Use of fertilizer magnesia for tobacco; Conn. Agr. Expt. Sta. Bull. 386 (1936) 578-585.

453. Pyriki, C.: Monosaccharide, Saccharose und Maltose im Tabak [Monosaccharides, saccharose, and maltose in tobacco]; Z. Untersuch. Lebensm. 82 (1941) 401-416.

454. Mach, F.: Experiments with manganese sulfate on tobacco; Ber. Grossh. Bad. Landw. Vers. Anst. Augustenb. (1910) 51-55.

455. Shmuk, A.A. and V. Shirokaya: An investigation of the fractionated resins of tobacco and Makhorka; Collected Works on the Chemistry of Tobacco, Vsesoyuznuii Inst. Tabachnoz Prom., Krasnodar, No.109(V) (1934) 77-87, see Chem. Abstr. 28 (1934) 6246.

456. Kincaid, R.R.: Toxicity of mercury vapor to germinating tobacco seeds; Plant Physiol. 11 (1936) 654-656.

457. Willaman, J.J.: Alkaloids of tobacco. Identification and determination; Ind. Eng. Chem. 44 (1952) 270-273.

458. Shmuk, A.A. and S. Kashirin: The methyl alcohol content of raw tobacco and tobacco products; U.S.S.R. State Inst. Tobacco Invest. Bull. 60 (1929) 29.

459. Lokschina, S.: Tobacco toxicosis; Soviet. Vestnik. Optalmol. 8 (1936) 464-468.

460. Arnon, D.I. and P.R. Stout: Molybdenum as an essential element for higher plants; Plant Physiol. 14 (1939) 599-602.

461. Kozlowska, A.: Changes caused by ammonium molybdate and virus diseases in plants; Bull. Intern. Acad. Polon. Sci. Classe Sci. Math. Nat. BI (1947) 109-165, see Chem. Abstr. 44 (1947) 4087.

462. Hunter, J.G. and O. Vergnano: Nickel toxicity in plants; Ann. Appl. Biol. 39 (1952) 279-284, see Soil Fert. XVI 2 (1952) 91.

463. Shira, K. and F. Kodaira: Studies of trace-element content in soil and green leaves Ni content; J. Sci. Soil Man. Japan 22 (1952) 175-176.

464. Späth, E. and E. Zajic: Über das $l$-Nornikotin. Mitteil III. Über Tabakbasen [About $l$-nornicotine. Part III. About tobacco bases]; Ber. Dtsch. Chem. Ges. 68 (1935) 1667-1670.

465. Späth, E., A. Wenusch, and E. Zajic: Tabak-Basen. V. Die Konstitution des Myosmins [Tobacco bases. V. The constitution of myosmine]; Ber. Dtsch. Chem. Ges. 69 (1936) 393-396.

466. Venkatarao, C., M. Narasingarao, and A. Venkateswarulu: Glyceride composition of tobacco seed oil; J. 
Indian Chem. Soc. 21 (1944) 249-252, see Chem. Abstr. 39 (1945) 2894.

467. Sabetay, S., G. Igolen, and L. Palfray: The odor of tobacco blossoms; Rev. Chim. Ind. (Paris) 50 (1941) 47-48, see Chem. Abstr. 38 (1944) 34133.

468. Betting, M.: Oxidase and peroxidase in tobacco; Med. Alg.-Proefsat. Java 27 (1910) 2; Chem. Zent. I (1910) 1043, see Chem. Abstr. 5 (1911) 740.

469. Roberts, E.A.H.: Investigations into the chemistry of the flue-curing of tobacco; Biochem. J. 35 (1941) 1289.

470. Sabetay, S. and J. Panouse: $d$-Rotatory $\beta$-methylvaleric acid in tobacco leaves; Compt. Rend. 225 (1947) 887-889, see Chem. Abstr. 42 (1948) 4527h.

471. Rayburn, C.H.: Chemical analysis of tobacco and tobacco smoke: Determination of some volatile phenols in cigarette smoke; $6^{\text {th }}$ Tobacco Chemists' Research Conference, Program Booklet and Abstracts, Vol. 6, Paper No. 10, 1952, p. 5.

472. Rayburn, C.H., W.R. Harlan, and H.R. Hanmer: Determination of volatile phenols in cigarette smoke; Anal. Chem. 25 (1953) 1419.

473. Cavalli, A.: Über die Verbrennungs-produkte des Tabaks [On the combustion products of tobacco]; Selmi 8 (1898) 25; Chem. Zent. 22(II) (1898) 235.

474. Traetta-Mosca, F.: Gli estratti di etere dei foglidel tobacco del Kentucky [The ether extracts of Kentucky tobacco leaves]; Gazz. Chim. Ital. 43 (1913) 440-445.

475.Kobel, M. and M. Scheuer: Der Kohlehydratmetabolismus in grünen Tabakblättern. Nachweis von Methylglyoxal als ein Zwischenprodukt des Metabolismus grüner Blätter [The carbohydrate metabolism of the tobacco leaf. Demonstration of methylglyoxal as an intermediate product in the metabolism of green leaves]; Biochem. Z. 216 (1929) 216-223, see Chem. Abstr. 24 (1930) 1664.

476. Cundiff, R.H, G.H. Greene, and A.H. Laurene: Column elution of humectants from tobacco and determination by vapor chromatography; Tob. Sci. 8 (1964) 163-168.

477. Kissling, R.: Handbuch der Tabakkunde [Handbook of tobacco information]; $3^{\text {rd }}$ Edition, Paul Parey, Berlin, Germany (1919).

478. Schöller, R.: 3-Pyridyläthylketon im Tabakrauch [3Pyridyl ethyl ketone in tobacco smoke]; Fachl. Mitt. Österr. Tabakregie 1935(3) 7.

479. Ribeiro, F.: The irritant action of cigar smoke; Arch. Hyg. Saude Publ. 2 (1937) 289-290, see Chem. Abstr. 33 (1939) 7959.

480. Shmuk, A.A.: Investigations of the polyphenol substances of tobacco (preliminary report); U.S.S.R. State Inst. Tobacco Invest. Bull. 69 (1930) 7.

481. Mendel, A.: Über die Mosaikkrankheit des Tabaks [On the mosaic disease of tobacco]; Landw. Vers.-Sta. 32 (1886) 451-467.

482. Brunton, T.L. and F.W. Tunnicliffe: On the physiological action of pyridine; J. Physiol. (London) $17(1894 / 1895)$ 272-276.

483. Warburg, H.: Nikotin- und Pyridingehalt des Tabakrauches bei Verwendung schwerer und leichter sowie nikotinfreier Tabake und ,nikotinunschädlicher“" Zigarren [Nicotine and pyridine content of tobacco smoke by use of strong and mild as well as "nicotinefree" and "nicotine-harmless" cigars]; Thesis,
Würzburg University (1906) pp. 1-38.

484. Pyatnitskii, M.P. and S.M. Kashirin: Studies on the chemical composition of tobacco smoke bases; Vsesoy. Inst. Tabach. Makharochnoi 133 (1937) 47-63.

485. Hofmann, E.: Quantitative spektralanalytische Bestimmung von Nikotin und Pyridin [Quantitative spectral determination of nicotine and pyridine]; Arch. Hyg. 128 (1942) 179-186.

486. Eulenburg, A.: Real-Encyclopädie der gesammten Heilkunde [Industrial encyclopedia of general medicines]; Vol . 24 (1900), Loebisch, Tobacco, p.19.

487. Dobrin, E.M.: A colorimetric method for determining nicotine and pyridine in tobacco; Vsesoyuzni Inst. Tabachnoi 104 (1933) 9-16.

488. Woodward, C.F., C.O. Badgett, and J.G. Kaufman: Chem-catalytic liquid-phase oxidation of nicotine, $\beta$ picoline and quinoline to nicotinic acid; Ind. Eng. Chem. 36 (1944) 544-546.

489. Woodward, C.F., F.A. Eisner, and P.G. Haines: Pyrolysis of nicotine to myosmine; J. Am. Chem. Soc. 66 (1944) 911-914.

490. Swain, M.L., A. Eisner, C.F. Woodward, and B.A. Brice: Ultraviolet absorption spectra of nicotine, nornicotine, and some of their derivatives; J. Am. Chem. Soc. 71 (1949) 1341-1345.

491. Sadykov, A.S., O.S. Ostroshchenko, and M.K. Yusupov: Syntheses from anabasine. VI. Amination of anabasine, $N$-methylanabasine and nicotine; $\mathrm{Zh}$. Obshsch. Khim. 23 (1953) 980-982, see Chem. Abstr. 48 (1954) $7616 f$.

492. Melsens, L.H.F.: Note sur la nicotine [A note on nicotine]; Ann. Chem. Pharm. 49/50 (1843) 353-359; Ann. Chim. Phys. 9 (1843) 465-472.

493. Melsens, L.H.F.: Note sur la nicotine [Note on nicotine]; Liebig Ann. Chem. 49 (1844) 353-359.

494.Heubel, E.: Experimental Beiträge zur Kenntnis der chemischen Eigenschaften und toxischen Wirkungen des Tabakrauche [Experimental contribution to the knowledge of the chemical properties and toxic effects of tobacco smoke]; Cent. Ed. Wissensch. 10 (1872) 641-647.

495. Kissling, R.: Betrachtungen über den Nikotingehalt des Cigarrenrauchs gemeinsam mit anderen giftigen Verbrennungsprodukten des Tabaks [Consideration of the nicotine content of cigar smoke considered simultaneously with other poisonous combustion products of tobacco]; Dingler's Polytech. J. 244 (1882) 64-71.

496. Kissling, R.: Betrachungen über den Nikotingehalt des Cigarrenrauchs gemeinsam mit der toxischen Wirkung der Verbrennungsprodukten des Tabaks [Consideration of the nicotine content of cigar smoke and the simultaneous consideration of the toxic action of the combustion products of tobacco]; Dingler's Polytech. J. 244 (1882) 234-246.

497. Lowenthal, M.: Untersuchungen über den Nikotingehalt des Tabakrauches [Research on nicotine content of tobacco smoke]; Medical Dissertation, Würzburg University (1892).

498. Kissling, R.: Nachweis von Nikotin im Tabakrauch [Evidence of nicotine in tobacco smoke]; Chem. Ztg. 22 (1898) 805.

499. Ludwig, E.: Über die Einführung nicotinarmer Cigarren [The introduction of low-nicotine cigars]; Österr. Sanitätswesen 14 (1902) 489-492. 
500. Bamberger, J.: Zur Hygiene des Rauchens [The hygiene of smoking]; Münch. Med. Wchnschr. 51 (1904) 1344-1345.

501. Paschkis, H.: Zur Hygiene des Rauchens [The hygiene of smoking]; Monat. Gesundheit. 22 (1904) 1-6.

502. Schmidt, F.: Der Nikotingehalt von Tabak und Tabakrauch [The nicotine content of tobacco and tobacco smoke]; Thesis, Würzburg University (1904) pp. 1-46.

503. Theodorovits, I.: The nicotine content of the smoke of different tobacco products; Magyar. Dohany. (Budapest) August 1 (1905) 5; August 16:2-3; September 1: 2-3; September 16: 2-3; October 1: 2-3.

504. Ratner, H.: Experimentelle Untersuchung über die physiologische Wirkung des Tabakrauches auf den Organismus [Experimental study of the physiological action of tobacco smoke on organisms]; Pflügers Arch. 113 (1906) 198-212.

505.Bitter, L.: Untersuchungen über die Bedeutung des Nikotins für die Stärke des Rauches [Investigation of the significance of nicotine for the strength effect of smoke]; Dissertation, Würzburg University (1907) pp. $1-30$.

506. Kuhles, J.: Investigation of the transition of nicotine from cigars and cigarettes with experiments on the absorption of nicotine from tobacco smoke in man; Dissertation, Würzburg University (1907) pp. 1-28.

507. Noda, S.: Beitrag zur Erklärung der Rauchwirkung (starke und milde Zigarren mit gleichem Nikotingehalt; Nikotingehalt von neuen und alten Pfeifen) [Contribution to the explanation of the action of smoke (strong and mild cigars with similar nicotine contents; nicotine content from new and old pipes)]; Thesis, Würzburg University (1908).

508. Toth, J.: The free nicotine content of tobacco smoke; Kiserletugyi Koslemenyek 11 (1908) 520-523.

509. Toth, J.: Über die Menge an freiem Nikotin im Tabakrauch [The free nicotine content of tobacco smoke]; Chem. Ztg. 33 (1909) 866-867.

510. Weger, G.A.: Was ist die Erklärung für die unterschiedliche Wirkung von Zigarren mit gleichem Nikotingehalt [What is the explanation of the different effect from cigars with similar nicotine contents]; Dissertation, Würzburg University (1909) pp. 1-23.

511. Fröhlich, A.: Die Toxikologie des Tabakrauches und des Nikotins [The toxicology of tobacco smoke and nicotine]; Deut. Med. Wchnschr. 37 (1911) 2268-2273.

512. Von Frankl-Hochwart, I.: Die nervösen Erkrankungen der Tabakraucher [Nervous diseases of the tobacco smoker]; Deut. Med. Wchnschr. 37 (1911) 2273-2274, 2321-2324.

513. Anonymous: The toxic factor in tobacco; Lancet 1912 (i) $944-947$

514. Favanger, H.: Experimentelle und klinische Untersuchungen der chronischen Tabakvergiftung [Experimental and clinical investigation of chronic tobacco poisoning]; Wien. Klin. Wchnschr. 27 (1914) 497-501.

515.Kütt, H.: Studien über die Vollständigkeit der Verbrennung des Nikotins im Tabakrauch [Studies on the completeness of combustion of nicotine in tobacco smoke]; Dissertation, Würzburg University (1915) pp. $1-40$.

516. Toth, H. and K. Dangelmajer: Über die Entnikotinisierung des Tabakrauches [Denicotinization of tobacco smoke]; Chem. Ztg. 40 (1916) 1013.

517. Silberbauer, R.: Physiologische Bestimmung des Nikotingehalts des Rauches von Zigarren, Zigaretten und Pfeifentabaken [Physiological determination of nicotine content of cigar, cigarette, and pipe tobacco smoke]; Medical Inaugural Dissertation, Erlangen, 1919.

518. Van Leeuwen, W.S.: On the nicotine content of the smoke of strong, mild and nicotine-free cigars; Arch. Exptl. Pathol. Pharmacol. 84 (1919) 282-316.

519. Asherson, H.: Nicotine and tobacco smoking; Chem. News J. Physiol.Sci. 120 (1920) 150.

520. Hahn, M. and H. Langer: Tabakrauch und Nikotin sowie eine biologische Methode für die quantitative Bestimmung des Nikotins [Tobacco smoke and nicotine and a biological method for the quantitative determination of nicotine]; Z. Hyg. Infektionskrankheit 90 (1920) 22-36.

521. Hirschfelder, A.D., A.E. Lange, and A.C. Feaman: The toxicity of tobacco smoke from cigars, cigarettes and pipe tobacco; Science 51 (1920) 21-22.

522. Bogner, H.: Die Bestimmung des Nikotingehaltes des Zigarettenrauches [Determination of the nicotine content of cigarette smoke]; Medical Inaugural Dissertation, Erlangen (1921).

523. Baumberger, J.P.: The nicotine content of tobacco smoke; J. Pharm. Exp. Therap. 21 (1923) 35-46.

524. Cerioli, G.: Analisi del tabacco [Analysis of tobacco]; Nuova Scelta di Opuscoli (Amoretti, Milano), Vol. II (1807) 250-258 [Work supported by the Academy of Science and Art, Milan].

525. Hermbstädt, S.H.: Bemerkungen über das Nicotianin [Remarks concerning nicotianine]; Abhandlungen der Königlichen Akademie der Wissenschaften, Phys. Klasse 7 (1822) 47-54, Berlin 1820/21.

526. Posselt, W. and L. Reimann: Chemische Untersuchungen des Tabaks und Darstellung des eigentümlichen wirksamen Prinzips dieser Pflanze [Chemical investigations of tobacco and preparation of the characteristic effective principles of this plant]; Geigers Magazin Pharm. 24 (1828) 138-161.

527. Barral, M.: On nicotine; Compt. Rend. 5 (1842) 167-168.

528. Ortigosa, M.: Über die Zusammensetzung des Nicotins und einiger seiner Verbindungen [On the composition of nicotine and several of its compounds]; Liebig Ann. 41 (1842) 114, see also Ortigosa, M.: Ann. Chem. Physiq. XLI (1842) 141.

529. Barral, M.: Note sur la formule de la nicotine [A note on the formula for nicotine]; Ann. Chem. Physiq. XLVI (1847) 345-353.

530. Vleminckx, J.F.: Experiments on the effects of nicotine; Gaz. Hôp. (July, 1851), see Abstr. in Am. J. Med. Sci. 23 (1852) 553.

531. Huber, C.: Vorläufige Notiz über einige Derivative des Nikotins [Preliminary notice of several derivatives of nicotine]; Justus Liebig Ann. Chem. 141 (1867) 271.

532. Jullien, J.: Étude sur la nicotine [Study on nicotine]; Dissertation thesis, Paris (1868) pp. 1-60.

533. Kopff, A.: De la nicotin [About nicotine]; Dissertation thesis, Strasbourg (1869) pp. 1-57.

534. Weidel, H.: Zur Kenntnis des Nikotins [Information on nicotine]; Justus Liebig Ann. Chem. 165 (1873) 328-349.

535. Von Laiblin, R.: Zur Kenntnis des Nikotins [Infor- 
mation on nicotine]; Ber. Dtsch. Chem. Ges. 10 (1877) 2136-2140.

536. Von Laiblin, R.: Über Nikotin und Nikotinsäure [Nicotine and nicotinic acid]; Justus Liebig Ann. Chem. 196 (1879) 129-182.

537. Prescott, A.B.: Contribution from the chemical laboratory of the University of Michigan. VIII. Estimation of nicotine in tobacco by E.T. Pease; J. Am. Chem. Soc. 2 (1880) 338-339.

538. Liebrecht, A.: Reduction des Nikotins [Reduction of nicotine]; Ber. Dtsch. Chem. Ges. 19 (1886) 2587-2598.

539. Gautier, A.: Comments on M. Moissan's communication; Compt. Rend. Acad. Sci. 115 (1892) 992-993.

540. Blau, F.: Zur Konstitution des Nikotins [On the constitution of nicotine]; Ber. Dtsch. Chem. Ges. 26 (1893) 628-633.

541. Etard, A.: Recherches sur la saturation des azotes de la nicotine et sur une acetylnicotine [On the saturation of the nitrogen of nicotine and on an acetylnicotine]; Compt. rend. 117, 170-173 (1893).

542.Pinner, A.: Über Nikotin. Die Konsitution des Alkaloids [Concerning nicotine. The constitution of the alkaloid]; Ber. Dtsch. Chem. Ges. 26 (1893) 292-305.

543.Pinner, A.: Über Nikotin. 6. Mitteilung [Concerning nicotine. Part 6]; Ber. Dtsch. Chem. Ges. 26 (1893) 765-771.

544.Étard, A.: Recherches sur la saturation des azotes de la nicotine et sur une acetylnicotine [Investigations on the saturation of the nitrogen of nicotine and on an acetylnicotine]; Bull. Soc. Chim. Franc. 3 (1894) 109-112.

545.Pinner, A.: Sur la saturation des azotes de la nicotine et sur l'acetylnicotine [On the saturation of the nitrogens of nicotine and of acetylnicotine]; Arch. Pharm. 233 (1895) 586-589.

546. Pictet, A. and P. Genequand: Über die Jodomethylate des Nikotins [On the iodomethylate of nicotine]; Ber. Dtsch. Chem. Ges. 30 (1897) 2117-2125.

547.Pictet, A. and A. Rotschy: Synthese des Nikotins [Synthesis of nicotine]; Ber. Dtsch. Chem. Ges. 37 (1904) 1225-1235.

548. Chuard, E. and R. Mellet; Variations de la proportions de nicotine dans les divers organs de la plant de tabac au cours de la végétation [Variations in the proportions of nicotine in the different organs of the tobacco plant during growth]; Compt. Rend. Acad. Sci. (Paris) 155 (1912) 293-295.

549. Popp, M. and J. Contzen: Bestimmung des Nikotins im Tabak und Tabakrauch [Estimation of nicotine in tobacco and tobacco smoke]; Chem. Ztg. 46 (1922) 1001-1002.

550. Rhode, H.: Nicotine content of the leaves and smoke of homegrown tobacco; Zeit. Untersuch. Nahrung. 45 (1923) 112-115.

551. Heiduschska, A. and F. Muth: Über Nikotin im Tabak [Nicotine in tobacco]; Pharm. Zentralhalle 68 (1927) 337-345.

552. Heiduschska, A. and F. Muth: Über Nikotin im Tabak [Nicotine in tobacco]; Pharm. Zentralhalle 68 (1927) 353-361.

553. Heiduschska, A. and F. Muth: Über Nikotin im Tabak [Nicotine in tobacco]; Pharm. Zentralhalle 68 (1927)
369-374.

554.Pfyl, B. and C.O. Schmitt: Zur Bestimmung des Nikotins im Tabak und Tabakrauch [On the determination of nicotine in tobacco and tobacco smoke]; Z. Untersuch. Lebensm. 54 (1927) 60-77.

555. Winterstein, E. and G. Trier: Alkaloide des Tabaks Nikotin [Tobacco alkaloids - Nicotine]; Monograph on Natural Bases 1 (1927) 335-336.

556. Harlan, W.R. and R.M. Hixon: Volatility of nicotine; Ind. Eng. Chem. 20 (1928) 723-724.

557.Popp, H.: Nikotinarme Tabake [Nicotine-poor tobacco]; Z. Planzen. 12A (1928) 334-344.

558. Heiduschska, A. and F. Muth: Über Nikotin im Tabak. II [Nicotine in tobacco. II]; Pharm. Zentralhalle 69 (1929) 305-307, 454.

559. Heiduschska, A. and F. Muth: Über Nikotin im Tabak. III [Nicotine in tobacco. III]; Pharm. Zentralhalle 70 (1929) 517-520.

560. Heiduschska, A. and F. Muth: Über Nikotin im Tabak. IV [Nicotine in tobacco. IV]; Pharm. Zentralhalle 70 (1929) 677-680.

561.Dixon, W.E.: The physiological effects of smoking; Practitioner 118 (1927) 20-28.

562. Winterstein, A. and E. Aronson: Beitrag zur Kenntnis der Tabaknutzung. Teil I [Contribution to the knowledge of tobacco uses. Part I]; Z. Hyg. Infektionskrank. 107 (1927) 487-497.

563. Schöller, R.: Nikotingehalt des Rauches feuchter und trockener Zigarren [Nicotine content of smoke of moist and dry cigars]; Fachl. Mitt. Österr. Tabakregie 1927/1928(2) 2-4.

564. Wenusch, A.: Nikotinbestimmungen in den Rauchgasen normaler und entnikotinisierter Zigarren [Nicotine determination in the smoke of normal and denicotinized cigars]; Fachl. Mitt. Österr. Tabakregie 1927/1928(1) 7-8.

565. Wenusch, A.: Nikotinmenge im Tabakrauch und Nikotinaufnahme durch den menschlichen Organismus beim normalen (intermittierenden) Rauchen [Nicotine content of tobacco smoke and nicotine uptake in the human organism during normal, puff by puff smoking] ; Fachl. Mitt. Österr. Tabakregie 1928(3) 8-11.

566. Winterstein, A. and E. Aronson: Beitrag zur Kenntnis der Tabaknutzung. Teil II. Über die Verteilung des Nikotins beim Tabakrauchen [Contribution to the knowledge of tobacco use. Part II. On the distribution of nicotine in tobacco smoking]; Z. Hyg. Infektionskrank. 108 (1928) 530-553.

567. Wenusch, A.: Beiträge zum Entnikotinisierungsproblem. Teil 1 [Contribution to the denicotinization problem. Part I]; Fachl. Mitt. Österr. Tabakregie 1929(2) 8-11.

568. Wenusch, A.: Beiträge zum Entnikotinisierungsproblem. Teil 2 [Contribution to the denicotinization problem. II]; Fachl. Mitt. Österr. Tabakregie 1929(2) 11-12.

569. Wenusch, A.: Beiträge zum Entnikotinisierungsproblem. Teil 3 [Contribution to the denicotinization problem. III]; Fachl. Mitt. Österr. Tabakregie 1929(3) 9-13.

570. Wenusch, A.: Beiträge zum Entnikotinisierungsproblem. Teil 4 [Contribution to the denicotinization problem. IV]; Fachl. Mitt. Österr. Tabakregie 1929(4) $5-10$. 
571. Winterstein, A. and E. Aronson: Beitrag zur Kenntnis der Tabaknutzung. Teil III. Die "hygienische" Zigarette [Contribution to the knowledge of tobacco uses. Part III. The "hygienic" cigarette]; Z. Hyg. Infektionskrank. 110 (1929) 644-653.

572. Winterstein, A. and E. Aronson: Über den Zigarettenrauch [On cigarette smoke]; Schweiz. Med. Wchnschr. 39 (1929) 550-552.

573. Bodnár, J.: Composition of tobacco smoke and denicotinization of tobacco; Termeszettudomanyi Kozlony 62 (1930) 397-404.

574. Bodnár, J.: Composition of tobacco smoke and denicotinization of tobacco; Termeszettudomanyi Kozlony 62 (1930) 443-446, see Chem. Abstr. 25 (1931) 2810.

575.Bolm, F.: Über den Rauch, Grenzwerte für Nikotin, und die Methode der Nikotinbestimmung nach Pfyl und Schmitt [On smoke, nicotine limit values, and the nicotine determination method of Pfyl and Schmitt]; Z. Untersuch. Lebensm. 59 (1930) 602-606.

576. Storp, W.: Die Entgiftung des Tabakrauchs durch Filtration [The detoxification of tobacco smoke by filtration]; Veröff. Gebiete Heeres-Sanität. 84 (1930) 165-189.

577. Wenusch, A.: Beiträge zum Entnikotinisierungsprobe. V [Contribution to the denicotinization test. V]; Fachl. Mitt. Österr. Tabakregie 1930(1) 13-19.

578. Wenusch, A.: Die Reaktion des Tabakrauches [The reaction of tobacco smoke]; Fachl. Mitt. Österr. Tabakregie 1930(2) 13-15.

579. Barta, L. and E. Toole: Mikrotitrimetrische Bestimmung des Nikotins im Tabakrauch [Microtitrimetric determination of nicotine in tobacco smoke]; Angew. Chem. 44 (1931) 682-683.

580. Ehrismann, O.: Untersuchungen über Nikotin und die Entnikotinisierung des Tabakrauches. II [Studies on nicotine and the denicotinization of tobacco smoke. II]; Z. Hyg. Infektionskrankh. 112 (1931) 698-707.

581. Frank, I.: The hygiene of tobacco smoke; Z. Fortbild. 28 (1931) 784-787.

582. Hahn, M. and O. Ehrismann: Untersuchungen über Nikotin und die Entnikotinisierung des Tabakrauches. I [Studies on nicotine and the denicotinization of tobacco smoke. I]; Z. Hyg. Infektionskrank. 112 (1931) 680-697.

583. Herrmann, K.: Untersuchungen über die Entnikotinisierung mittels der Bonikot Methode [Research on denicotinization by the Bonicot method]; Med. Welt (1931) 1564.

584. Kovalenko, E.: The nicotine content of tobacco smoke; in: Research on the chemistry of tobacco, edited by A.A. Shmuk, Gosudarstvennyi Inst. Tabakovedeniya Publ. No. 80 (1931) 77-85.

585. Pyriki, C.: Über das Auftreten von Nikotin im Zigarettenrauch [On the occurrence of nicotine in cigarette smoke]; Z. Angew. Chem. Zent. Tech. Chem. 44 (1931) 553-554.

586. Pyriki, C.: Über das Auftreten von Nikotin im Zigarettenrauch [On the occurrence of nicotine in cigarette smoke]; Z. Untersuch. Lebensm. 62 (1931) 95-99.

587. Van Druten, A.: Wirkung der "Bonikot" Injektion auf den Nikotingehalt von Zigarrenrauch [Influence of "Bonicot" injection on the nicotine content of cigar smoke]; Z. Untersuch. Lebensm. 62 (1931) 633-647.

588. Wenusch, A.: Beitrag zur Bestimmung des Nikotins in
Rauchgasen. I [Contribution to the determination of nicotine in smoke. I]; Fachl. Mitt. Österr. Tabakregie 1931(1) 1-8.

589. Wenusch, A.: Beitrag zur Bestimmung des Nikotins in Rauchgasen. II [Contribution to the determination of nicotine in smoke. II]; Fachl. Mitt. Österr. Tabakregie 1931(2) 1-3.

590. Wenusch, A.: Über die Stärke der Tabakfabrikate [On the strength of tobacco products]; Fachl. Mitt. Österr. Tabakregie 1931(3) 1-10.

591. Heiduschska, A. and E. Post: Über die Bestimmung des Nikotins im Tabakrauch [Determination of nicotine in tobacco smoke]; Pharm. Zentralhalle 73 (1932) 529-530.

592. Kissling, R.: Über die Entfernung des Nikotins aus dem Tabakrauch während des Rauchens [Removal of nicotine from tobacco smoke during smoking]; Chem. Ztg. 56 (1932) 31.

593. Kissling, R.: Über die Entnikotinisierung des Tabakrauchs während des Rauchens [Denicotinization of tobacco smoke during smoking]; Chem. Ztg. 56 (1932) 822-823.

594. Margasinski, Z.: Tobacco smoke; Przemysl Chem. 16 (1932) 224-225, 250-251.

595. Michalowsky, E.H.: Comparative experiments on the influence of "Bonicot" on the nicotine content of cigar and cigarette smoke; Münch. Med. Wochnschr. 79 (1932) 827-828.

596. Molinari, E.: Über den Einfluß der Luftfeuchtigkeit auf den Nikotingehalt des Tabakrauches [The influence of atmospheric moisture on the nicotine content of tobacco smoke]; Fachl. Mitt. Österr. Tabakregie 1932 (2) 4-7.

597. Nagy, V.L.: How much nicotine gets into cigarette smoke? Biochem. Z. 254 (1932) 94-96.

598. Nagy, V.L.: Microdetermination of nicotine in tobacco smoke; Biochem. Z. 249 (1932) 404-408.

599. Paffgen, J.: Verfahren zur Abtrennung von giftigen Bestandteilen aus dem Tabakrauch [Process for the separation of the poisonous constituents of tobacco smoke]; German Patent No. 549, 413 (1932).

600.Pyriki, C.: Über die Aufnahme des Nikotins beim Zigarettenrauchen [On the absorption of nicotine in cigarette smoking]; Z. Untersuch. Lebensm. 64 (1932) 163-171.

601.Pyriki, C.: Über das Auftreten von Nikotin im Zigarettenrauch. II [On the occurrence of nicotine in cigarette smoke. II]; Z. Untersuch. Lebensm. 64 (1932) 263-277.

602.Pyriki, C.: Der Genuss des Tabakrauches und die Bedeutung des Nikotins [The enjoyment of tobacco smoke and the importance of nicotine]; Prakt. Ärzt. 29 (1932) 45-47.

603. Schaarschmidt, A., H. Hoffmeier, and P. Nowak: Applicability of absorption agents for removing poisons from tobacco smoke; Chem. Ztg. 56 (1932) 911-913.

604. Schlossmann, H. and M. Schlesinger: Wird der Nikotingehalt des Zigarrenrauches durch Bonicot vermindert? [Is the nicotine content of cigar smoke decreased by Bonicot?] Klin. Wchnschr. 11 (1932) 371-372.

605. Shmuk, A.A., S.M. Kashirin, and A.P. Smirnov: Denicotinizing cigarettes by filtration of the smoke; Vsesoyuznuii Inst. Tabaknoi Prom. 90 (1932) 66-71. 
606. Skumburdis, K. and R. Kissling: Zur Frage der Denikotinisierung des Tabakrauches während des Rauchens [The question of denicotinization of tobacco smoke while smoking]; Chem. Ztg. 56 (1932) 208.

607. Traube, L.: Über die Entfernung des Nikotins aus dem Tabakrauch während des Rauchens [Removal of nicotine from tobacco smoke during smoking]; Chem. Ztg. 56 (1932) 963.

608. Van Druten, A.: Diskussion der Arbeit von M. Hahn und O. Ehrismann: Nikotin und die Entnikotinisierung des Tabakrauches. Teil I [Discussion of the work of M. Hahn and O. Ehrismann: Nicotine and the denicotinization of tobacco smoke. Part I]; Z. Hyg. Infektionskrank. 113 (1932) 754-755.

609. Waser, E. and M. Stähli: Untersuchung des Tabakrauches. I [Investigation of tobacco smoke. I]; Z. Untersuch. Lebensm. 64 (1932) 470-485.

610.Waser, E. and M. Stähli: Untersuchung des Tabakrauches. II [Investigation of tobacco smoke. II]; Z. Untersuch. Lebensm. 64 (1932) 569-573.

611. Nagy, V.L. and S. Dickmann: Volumetric determination of very small amounts of nicotine; Magy. Gyog. Tarsasag Ertesit. 9 (1933) 210-215.

612. Nagy, V.L. and S. Dickmann: Volumetrische Bestimmung kleinster Nikotinmengen [Volumetric determination of very small amounts of nicotine]; $Z$. Anal. Chem. 94 (1933) 12-17.

613. Braun, K.: Zur Gehaltsbestimmung von Nikotin im Tabak und im Tabakrauch [Determination of nicotine content in tobacco and in tobacco smoke]; Chem. Ztg. 55 (1930) 354-355.

614. Braun, K.: Erfahrung bei der Nikotinbestimmung im Tabak und im Tabakrauch [Knowledge of the determination of nicotine in tobacco and tobacco smoke]; Pharm. Zentralhalle 71 (1930) 209-213.

615. Faitelowitz, A.: Über die Bedeutung der Acidität des Tabaks für seine hygienische Beurteilung [On the significance of the acidity of tobacco for its hygienic evaluation]; Z. Untersuch. Lebens. 60 (1930) 518-523.

616. Harlan, W.R. and R.M. Hixon: Catalytic reduction of nicotine and metanicotine; J. Am. Chem. Soc. 52 (1930) 3385-3388.

617. Heiduschska, A.: Erfahrung bei der Nikotinbestimmung im Tabak und im Rauch [Knowledge of the determination of nicotine in tobacco and in smoke]; Pharm. Zentralhalle 71 (1930) 305-306.

618. Windus, W. and C.S. Marvel: The reduction of nicotine and some derivatives of hexa- and octahydronicotine; J. Am. Chem. Soc. 52 (1930) 2543-2546.

619. Auprecht: Investigation of new drugs, antiseptics and curative agents; Pharm. Zeit. 76 (1931) 427-448.

620. Tonn, O.: Results of tests on denicotinizing tobacco by the "Nicoton" and "Bonicot" methods; Apotheker Ztg. 46 (1931) 1393-1395.

621.Post, E.: Beitrag zur Kenntnis des Nikotins [Contribution to the knowledge of nicotine]; Thesis, Leipzig (1932) pp. 1-58.

622. Waser, E.: Die Gefahren des Nikotins [The danger of nicotine]; Schweiz. Med. Wchnschr. 13 (1932) 249-254.

623.Hofmann, E.: Semimicro- and microcolorimetric determination of nicotine in tobacco and tobacco smoke; Biochem. Z. 260 (1933) 26-33.

624. Toole, E.: The polarimetric determination of nicotine in tobacco and tobacco smoke; Z. Anal. Chem. 93 (1933) 188-194.

625. Koperina, A.W. and S. Kalibab: Denicotinized cigarettes; Tabakhnaya Promysh. 1935(3) 34-35.

626. Späth, E. and F. Kesztler: Über neue Tabakbasen. II. Ein Vereinfachung der Pictetschen Nikotinsynthese [On new tobacco bases. II. A simplification of the nicotine synthesis by Pictet]; Ber. Dtsch. Chem. Ges. 68 (1935) 494-497.

627. Wenusch, A.: Über die Anreicherung von Nikotin im Stummel von Zigaretten aus Orient Tabaken [Accumulation of nicotine in the butt of cigarettes made with Oriental tobacco]; Z. Untersuch. Lebensm. 70 (1935) 536-537.

628. Busbey, R.L. and N.E. McIndoo: A bibliography of nicotine, Part I. Chemistry of nicotine; Division of Insecticide Investigations and Division of Control Investigations; USDA, 1936.

629. Busbey, R.L. and N. E. McIndoo: A bibliography of nicotine; Part II. Chemistry of nicotine; Division of Insecticide Investigations and Division of Control Investigations; USDA, 1936.

630. Malaquin, A.: La nicotine dans les tabacs et leurs fumées [Nicotine in tobaccos and their smokes]; Douai (France) (1936) 1-78.

631. McIndoo, N.E. R.C. Roark, and R.L. Busbey: A bibliography of nicotine, Part II, Section 1: The insecticidal uses of nicotine and tobacco; USDA, 1936.

632. McIndoo, N.E. R.C. Roark, and R.L. Busbey: A bibliography of nicotine, Part II, Section 2: The insecticidal uses of nicotine and tobacco; USDA, 1936.

633. McIndoo, N.E. R.C. Roark, and R.L. Busbey: A bibliography of nicotine, Part II, Section 3: The insecticidal uses of nicotine and tobacco; USDA, 1936.

634. Späth, E. and E. Kainrath: Über neue Tabakalkaloide. XV. Über die Pictetsche Nikotinsynthese [On new tobacco alkaloids. XV. About the nicotine synthesis by Pictet]; Ber. Dtsch. Chem. Ges. 70 (1937) 1276-1281.

635. Mulinos, M.G. and J.R. Cockrill: The filtering power of cigarette tobacco; Arch. Internat. Pharm. Dyn. 58 (1937/1938) 200-207.

636. Molinari, E.: Über Stickstoffkörper des Tabaks und Tabakrauches [The nitrogen compounds of tobacco and tobacco smoke]; Fachl.. Mitt. Österr. Tabakregie 1938(3) 12-15.

637. Avens, A.W. and G. W. Pearce: Silicotungstic acid determination of nicotine. Errors involved and a new technique for steam-distillation of nicotine; Ind. Eng. Chem. 11 (1939) 505-508.

638. Shmuk, A.A. and A. Borozdina: Determination of the content of nicotine and anabasine together; J. App. Chem. 12(10) (1939) 1582.

639. Reif, G.: Nikotinarmer und nikotinfreier Tabak [Nicotine-poor and nicotine-free tobacco]; Z. Ärzt. Fortbild. 37 (1940) 374-375.

640.Dawson, R.F.: Nicotine synthesis in excised roots; Am. J. Bot. 29 (1942) 813-815

641. Haag, H.B. and P.S. Larson: Some chemical and pharmacological observations on "low nicotine" tobacco; Science 97 (1943) 187-188.

642. Pyriki, C.: Beitrag zur Standardisierung der Nikotinbestimmung [Contribution to the standardization of nicotine determination]; Acta Nicotiana 2 (1943) 116-121.

643.Larson, P.S. and H.B. Haag: Quantitative 
determination of nicotine and nornicotine in mixtures; Ind. Eng. Chem., Anal. Ed. 16 (1944) 86-90.

644. Clemo, G.R. and G.A. Swann: A contribution to the study of nicotine and the synthesis of 7-azaindole derivatives; J. Chem. Soc. (1945) 603-607.

645.Haag, H.B., P.S. Larson, and J.K. Finnegan: Studies on factors influencing the toxicity of nicotine; J. Pharm. Exp. Ther. 85 (1945) 356-362.

646.Larson, P.S., H.B. Haag, and J.K. Finnegan: On the relative toxicity of nicotine and nornicotine; Proc. Soc. Exp. Biol. 58 (1945) 231-232.

647. Brice, B.A.: Ultraviolet absorption spectra of nicotine, nornicotine, and some of their derivatives; $2^{\text {nd }}$ Tobacco Chemists' Research Conference, Program Booklet and Abstracts, Vol. 2, Paper No. 4, 1948.

648.Dawson, R.F.: Method for the quantitative determination of nicotine and nornicotine. A progress report; $2^{\text {nd }}$ Tobacco Chemists' Research Conference, Program Booklet and Abstracts, Vol. 2, Paper No. 1, 1948.

649. Griffith, R.B. and R.N. Jeffrey: Improved steamdistillation apparatus: Application to determination of nicotine in green and dry tobacco; Anal. Chem. 20 (1948) 307-311.

650. Willits, C.O.: Determination of nicotine by ultraviolet absorption; $2^{\text {nd }}$ Tobacco Chemists' Research Conference, Program Booklet and Abstracts, Vol. 2, Paper No. 5, 1948.

651. Porter, W.L., J. Naghski, and A. Eisner: Partition of tobacco alkaloids and some nicotine transformation products on a paper sheet support; Arch. Biochem. 24 (1949) 461-463, see Chem. Abstr. 44 (1950) 4637f.

652. Swain, M.L., A. Eisner, C.F. Woodward, and B.A. Brice: Ultraviolet absorption spectra of nicotine, nornicotine, and some of their derivatives; J. Am. Chem. Soc. 71 (1949) 1341-1345.

653. Dawson, R.F.: Formation of nornicotine from nicotine in burley tobacco; $4^{\text {th }}$ Tobacco Chemists' Research Conference, Program Booklet and Abstracts, Vol. 4, Paper No. 2, 1950.

654.Pyriki, C.: Über das Auftreten von Nikotin im Zigarettenrauch. III [On the occurrence of nicotine in cigarette smoke. III]; Z. Untersuch. Lebensm. 65 (1933) 566-571.

655. Schlossmann, H.: The nicotine content in the smoke of cigarettes containing low-nicotine, nicotine-detoxified, and nicotine-free tobaccos; Klin. Wchnschr. 12 (1933) 1255-1258.

656. Stähli, M.: Research on tobacco smoke; Thesis, Eidgenossichen Technischen Hochschule, Zurich, Switzerland, Publ. No. 757 (1933) pp. 1-53.

657. Graham, J.H.: On the trail of nicotine during the smoking process; Am. J. Pharm. 106 (1934) 256-261.

658. Koperina, A.W.: The balance of nicotine in the smoking of tobacco in cigarettes; Tabakhnaya Promysh. 1934(5) 37-40.

659. Koperina, A.W. and A. Shageeva: Transfer of nicotine into the smoke for different conditions of burning Makhorka and Makhorka dust; Tabakhnaya Promysh. 1934(1) 15-19.

660. Nagy, V.L. and L. Barta: Über den Nikotingehalt des Zigarren- und Pfeifenrauchs [Nicotine content of cigar and pipe smoke]; Angew. Chem. 47 (1934) 214-215.

661. Preiss, W.: Über die Verringerung des Nikotingehalts im Rauch durch Fumasan-Stäbchen [On decreasing the nicotine content of smoke by Fumasan sticks]; Pharm. Zentralhalle 75 (1934) 501-503.

662. Pyriki, C.: Über das Auftreten von Nikotin im Zigarettenrauch. IV [On the occurrence of nicotine in cigarette smoke. IV]; Z. Untersuch. Lebensm. 68 (1934) 420-431.

663. Pyriki, C.: Über die Verteilung des Nikotins beim Rauchen von Zigaretten [Distribution of nicotine in the smoking of cigarettes]; Chem. Ztg. 58 (1934) 279.

664.Pyriki, C.: Über die Verteilung des Nikotins beim Rauchen von Zigaretten [On the distribution of nicotine with the smoking of cigarettes]; Chem. Ztg. 58 (1934) 543.

665. Waser, E. and M. Stähli: Untersuchung des Tabakrauches. III [Investigation of tobacco smoke. III]; Z. Untersuch. Lebensm. 66 (1933) 354-362.

666. Wenusch, A.: Über die Bestimmung des Nikotins im Tabakrauch [Determination of nicotine in tobacco smoke]; Biochem. Z. 271 (1934) 280-284.

667. Wenusch, A.: Über die Verteilung des Nikotins beim Rauchen von Zigaretten [On the distribution of nicotine with the smoking of cigarettes]; Chem. Ztg. 58 (1934) 206-207.

668. Wenusch, A.: Über die Verteilung des Nikotins beim Rauchen von Zigaretten [On the distribution of nicotine with the smoking of cigarettes]; Chem. Ztg. 58 (1934) 310.

669. Wenusch, A.: Über die Verteilung des Nikotins beim Rauchen von Zigaretten [On the distribution of nicotine with the smoking of cigarettes]; Chem. Ztg. 58 (1934) 543.

670. Wenusch, A.: Theoretische Grundlagen zur Kenntnis der Nikotinverteilung beim Rauchen von Zigaretten [Theoretical basis for information on the nicotine distribution in cigarette smoking]; Z. Untersuch. Lebensm. 68 (1934) 412-420.

671. Wulfert, K.: The determination of nicotine in the smoke of Norwegian and foreign tobaccos; Tidsskr. Kjemi, Bergvesen 14 (1934) 128-131.

672. Wulfert, K.: The determination of nicotine in the smoke of Norwegian and foreign tobaccos; Tidsskr. Kjemi, Bergvesen 14 (1934) 140-143.

673. Bodnár, J., V.L. Nagy, and S. Dickmann: Uptake of nicotine by the human organism during smoking: Fate of nicotine; Biochem. Z. 276 (1935) 317-322.

674. Bodnár J, V.L. Nagy, and T. Vecsey: Adsorption of nicotine from tobacco smoke by different adsorbents; Pharm. Zentralhalle 76 (1935) 657-661.

675. Jensen, C.O. and D. Haley: Studies on the nicotine content of cigarette smoke; J. Agr. Res. 51 (1935) 267-276.

676.Preiss, W.: Wirkung von Zellulose-Filtereinlagen in Zigaretten auf den Nikotingehalt des Rauches [Action of cellulose filters in cigarettes on the nicotine content of smoke]; Pharm. Zentralhalle 76 (1935) 313-316.

677.Pyriki, C.: Über das Auftreten von Nikotin im Zigarettenrauch. V [On the occurrence of nicotine in cigarette smoke. V]; Z. Untersuch. Lebensm. 70 (1935) 527-535.

678.Pyriki, C.: Über die Anreicherung des Nikotins in Zigarettenstummeln [On the concentration of nicotine in cigarette butts]; Pharm. Zentralhalle 76 (1935) 513-517.

679. Heiduschska, A.: Über die Bestimmung des Nikotins im Tabakrauch [Determination of nicotine in tobacco 
smoke]; Pharm. Zentralhalle 77 (1936) 780-782.

680. Wenusch, A.: Der Nikotingehalt des Hauptstromrauchs dicker und dünner Zigaretten [The nicotine content of the mainstream smoke of thick and thin cigarettes]; $\mathrm{Z}$. Untersuch. Lebensm. 72 (1936) 213-218.

681. Wenusch, A.: Über die Bestimmung des Nikotins im Tabakrauch [Determination of nicotine in tobacco smoke]; Pharm. Zentralhalle 77 (1936) 645-646.

682. Bailey, C.F. and A.W. Petre: The modern cigarette industry; Ind. Eng. Chem. 29 (1937) 11-19.

683.Derr, R.B., A.H. Riesmeyer, and R.B. Unangst: Removal of undesirable constituents from tobacco smoke; Ind. Eng. Chem. 29 (1937) 771-776.

684. Pierce, L.H.: The absorption of nicotine in cigarette smoking; J. Pharmacol. Exptl. Therap. 60 (1937) 114-115.

685.Pyriki, C.: Über den Zigarettenrauch und dessen Nikotin [On cigarette smoke and its nicotine]; Pharm. Zentralhalle 78 (1937) 313-318.

686. Wenusch, A.: Über die Bestimmung des Nikotins im Tabakrauch [The determination of nicotine in tobacco smoke]; Z. Untersuch. Lebensm. 74 (1937) 46-51.

687. Wenusch, A.: Über den Einfluss der Schnittbreite auf den Übergang des Nikotins in den Hauptstromrauch [The influence of cut width on the passage of nicotine into mainstream smoke]; Z. Untersuch. Lebensm. 75 (1937) 182-184.

688. Justen-Müller, E.: Contribution to the study of cigarette and cigar smoke; J. Pharm. Chim. 28 (1938) 430-435.

689. Nagy, V.L.: Mikrobestimmung der TabakrauchAlkalität [Microdetermination of tobacco smoke alkalinity]; Pharm. Zentralhalle 79 (1938) 505-508.

690.Haag, H.B.: The physiologic activity of cigarette smoke solutions as related to their nicotine content; J. Lab. Clin. Med. 25 (1939/1940) 610-618.

691. Haag, H.B. and R.C. Neale: Comparative toxicity of nicotine and some of its salts; J. Pharm. Exp. Ther. 69 (1940) 289.

692.Haag, H.B., J.H. Weatherby, and R.C. Neale: Studies on nicotine; Bull. Med. Coll. Virginia 37 (1940) 1-4.

693. Wenusch, A.: Über die Nikotinaufnahme beim Tabakrauchen [Nicotine assimilation by tobacco smoking]; Med. Klin. 36 (1940) 1159-1161.

694. Wenusch, A.: Tabakuntersuchungen. V. Der Einfluss der Wärmefermentation auf den "Nikotinschub" [Tobacco investigations. V. The influence of warm fermentation on "nikotinschub"]; Z. Untersuch. Lebensm. 79 (1940) 265-267.

695. Wenusch, A.: Über die Nikotinaufnahme beim Tabakrauchen [On the uptake of nicotine from tobacco smoke]; Med. Klin. 36 (1940) 1159-1161.

696. Willits, C.O., M.L. Swain, J.A. Connelly, and B.A. Brice: Spectrophotometric determination of nicotine; Anal. Chem. 22 (1950) 430-433.

697. Weybrew, J.A., T.J. Mann, and R.J. Monroe: The alkaloid components of hybrids between $N$. tabacum and N. sylvestris; $6^{\text {th }}$ Tobacco Chemists' Research Conference, Program Booklet and Abstracts, Vol. 6, Paper No. 3, 1952, pp. 9-11.

698. Cundiff, R.H. and P.C. Markunas: A rapid titrimetric procedure for the determination of nicotine, nornicotine, and total alkaloids in tobacco and tobacco products; $7^{\text {th }}$ Tobacco Chemists' Research Conference, Program Booklet and Abstracts, Vol. 7, Paper No. 3,
1953, p. 4.

699. Pierce, L.H.: The absorption of nicotine in cigarette smoking; J. Lab. Clin. Chem. 26 (1941) 1322-1325.

700. Wenusch, A.: Letzte Forschungsergebnisse über Tabak, Tabakrauch und die Nikotinadsorption beim Tabakrauchen [Latest research results on tobacco, tobacco smoke, and nicotine absorption from tobacco smoking]; Österr. Chem. Z. 44 (1941) 228-233.

701. Wenusch, A.: Über die absoluten Nikotinmengen, die während des Rauchens aufgenommen werden [Absolute amounts of nicotine ingested during tobacco smoking]; Forschung. Fortschritte 18 (1942) 77-78.

702. Wenusch, A.: Über die absoluten Mengen des beim Rauchen aufgenommenen Nikotins [On the absolute quantity of nicotine taken up by smoking]; Chem. Ztg. 66 (1942) 254-255.

703. Wenusch, A.: Versuche über einen biologischen Abbau des Nikotins [Experiment on the biological degradation of nicotine]; Z. Untersuch. Lebensm. 84 (1942) 329-331.

704. Wenusch, A.: Weitere Untersuchungen über einen biologischen Abbau des Nikotins [Additional research on the biological degradation of nicotine]; $\mathrm{Z}$. Untersuch. Lebensm. 84 (1942) 498-501.

705. Pyriki, C.: Über die Nikotinanreicherung in Zigarettenstummeln und seine Beziehung zur Reaktion des Hauptstromrauchs [Nicotine accumulation in cigarette stubs and its relation to reaction of the main smoke stream]; Z. Untersuch. Lebensm. 85 (1943) 337-346.

706. Woodward, C.F., C.O. Badgett, and J.J. Willaman: Nicotine to nicotinonitrile catalytic vapor-phase oxidation; Ind. Eng. Chem. 36 (1944) 540-544.

707. McCormick W.E. and M. Smith: Determination of nicotine in the air; Ind. Eng. Chem. (Anal.) 18 (1946) 508-512.

708. Finnegan, J.K., P.S. Larson, and H.B. Haag; Studies on cigarette smoke irritation. II. The role of nicotine; Proc. Soc. Exptl. Biol. Med. 65 (1947) 200-202.

709. Sollmann, T.: A manual of pharmacology and its application to the therapeutics of toxicology; $7^{\text {th }}$ Edition, Philadelphia, PA (1948) 351-352.

710. Dabrowska, O.: Nicotine, resins, reducing agents in the smoke of Polish cigarettes from different fluecured and Turkish type tobaccos; Prace Tytoniowe Zeszyt (1949) 149-165; Rocznick Naut. Polniczych. (1949) 53.

711.Ling, H.W. and C.B. Wynn Parry: The amount of nicotine absorbed in smoking; Brit. J. Pharmacol. Chemotherapy 4 (1949) 313-314.

712. Wenusch, A.: What hopes can be based on nicotine filters? Rev. Internat. Tabacs 24 (1949) 136, 147.

713. Rayburn, C.H.: Influence of certain variables on the composition of cigarette smoke; Proc. Res. Conf. Chemistry of Tobacco (1950) 10-11.

714. Marchand, J. and J. Renard: Nicotine content in cigarette smoke; Papeterie 73 (1951) 263-265, 267.

715. Vettors, L.: Determination of nicotine in tobacco smoke; Ann. Assoc. Brazil. Quim. 10 (1951) 78-82.

716. Greenberg, L.A., D. Lester, and H.W. Haggard: The absorption of nicotine in smoking; J. Pharmacol. Exptl. Therap. 104 (1952) 162-167.

717. Staub, M. and H. Furrer: The determination of nicotine and tar in tobacco smoke; Mitt. Gebiet. Lebensm. Hyg. 44 (1953) 371-377.

718. Staub, M. and H. Furrer: The testing of nicotine filters; 
Mitt. Gebiet. Lebens. Hyg. 44 (1953) 472-474.

719. Wahl, R. and O. Heil: Die Entwicklung einer neuen Rauchapparatur unter besonderer Berücksichtigung der Strömungsverhältnisse [Development of a new smoking apparatus with special regard to the gas flow relationships]; in: Sonderheft anlässlich des 25jährigen Bestehens der Bundesanstalt für Tabakforschung in Forchheim [Volume devoted to the 25 years' existence of the Institute for Tobacco Study in Forchheim] (1953) 16-20.

720. Wolman, W. and R.R. Stark: A study of cigarettes, cigarette smoke, and filters. I. Filter-tip cigarettes; J. Am. Med. Assoc. 152 (1953) 917-920.

721. Wolman, W. and R.R. Stark: A study of cigarettes, cigarette smoke, and filters. II. Special low-nicotine cigarettes; J. Am. Med. Assoc. 152 (1953) 1035-1036.

722. Rayburn, C.H., W. R. Harlan, and H.R. Hanmer: Rearrangement of nicotine oxide; J. Am. Chem. Soc. 72 (1950) 1721-1723.

723. Pinner, A.: Über Nikotin [Concerning nicotine]; Ber. Dtsch. Chem. Ges. 28 (1885) 456.

724. Wenusch, A.: Über die Menge an nichtflüchtigen Tabakrauchbestandteilen und die Löslichkeit von Nikotinsalzen in verschiedenen Lösungsmitteln [The amount of nonvolatile tobacco smoke constituents and the solubility of nicotine salts in different solvents]; Z. Untersuch. Lebensm. 74 (1937) 43-46.

725.Hermbstädt, S.F.: Nicotianin oder Tabak-Kampfer [Nicotianine or tobacco-camphor]; Schw. 31, 442; Abstr. Berz. Jahresber (1823) 113.

726. Pictet, A. and P. Crépieux: Über die Hydrierung des Nicotyrine [On the hydrogenation of nicotyrine]; Ber. Dtsch. Chem. Ges. 31 (1898) 2018-2022.

727. Wibaut, J.P. amd J. Overhoff: The catalytic hydrogenation of nicotine. A suitable method for the preparation of $N$-methyl-(3-pyridyl)-2-pyrrole (3,2'nicotyrine); Rec. Trav. Chem. Pays-Bas 47 (1928) 935-939.

728. Wibaut, J.P. and J.T. Hackman: Reduction of 3,2'nicotyrine to dihydronicotyrine and inactive nicotine disproportionation of dihydronicotyrine; Rec. Trav. Chem. Pays-Bas 51 (1932) 1157-1165.

729. Wenusch, A.: Über das Auftreten von Nikotyrin im Tabak [On the occurrence of nicotyrine in tobacco]; Biochem. Z. 275 (1935) 361.

730. Haag, H.B.: A contribution to the pharmacology of anabasine; J. Pharm. Exp. Ther. 48 (1933) 95-104.

731.Smith, C.R.: Neonicotine and isomeric pyridylpiperidines; J. Am. Chem. Soc. 53 (1931) 277-281.

732. Smith, C.R.: Identity of neonicotine and the alkaloid anabasine; J. Am. Chem. Soc. 54 (1932) 397-399.

733. Olchansky, M.I.: Le nouvel alcaloide anabasine, ses propriétés toxiques et curatives [The novel alkaloid anabasine, its toxic and curative properties]; Proc. $15^{\text {th }}$ Int. Physiol. Cong. (1935) 276-277.

734. Smith, C.R.: Occurrence of anabasine in Nicotiana glauca R. Grah (Solanaceae); J. Am. Chem. Soc. 57 (1935) 959-960.

735. Smith, H.H. and C.R. Smith: Alkaloids in certain species and interspecific hybrids of Nicotiana; J. Agr. Res. 65 (1942) 347-359.

736. Dubinin, B.M. and G.V. Chelintsev: Pyrolysis of anabasine; Zhur. Obshch. Khim. 16 (1946) 105-108.

737. Matveev, B.V.: Catalytic oxidation of anabasine; J.
Gen. Chem. USSR 17 (1947) 482-484.

738. Sadykov, A.S. and N. Ashrapova: Syntheses from anabasine. III. Condensation of aliphatic oxides with anabasine; Zhur. Obshsch. Khim. 17 (1948) 1212-1215.

739. Späth, E. and E. Zajic: Über neue Tabakbasen. III. Über das $l$-Nornikotin [On new tobacco bases. III. About $l$-nornicotine]; Ber. Dtsch. Chem. Ges. 68 (1935) 1667-1670.

740. Wenusch, A.: Über das Nornikotin [Concerning nornicotine]; Pharm. Zentralhalle 77 (1936) 141-143.

741.Späth, E., L. Marion, and E. Zajic: Über neue Tabakbasen. IV. Synthese des $l$-Nornikotin [On new tobacco bases. IV. Synthesis of $l$-nornicotine]; Ber. Dtsch. Chem. Ges. 69 (1936) 251-255.

742. Chen, K.K., C.L. Rose, and E.B. Robins: Toxicity of nicotinic acid; Proc. Soc. Exp. Biol. 38 (1938) 241-245.

743. Gottscho, A. and W.G. Frankenburg: A comprehensive method of analysis of the nitrogen compounds of tobacco and some changes occurring during fermentation of cigar tobacco; $6^{\text {th }}$ Tobacco Chemists' Research Conference, Program Booklet and Abstracts, Vol. 6, Paper No. 8, 1952, p. 8.

744.Pictet, A.: $N$-Methylpyrrolidin aus Nikotin $[N-$ Methylpyrrolidine from nicotine]; Ber. Dtsch. Chem. Ges. 38 (1905) 1951-1952.

745. Von der Porten, I.: Über Tabakpapier [About tobacco paper]; Dingler's Polytech. 146 (1857) 240, 435-436, also see Suter, J.S. and G.M. Palmer: Improvements in wrappers for cigars; U.S. Patent No. 21,704 (October $5,1858)$

746. Koperina, A.W.: Research on tobacco chemistry. VII. The resins of tobacco smoke; Biochem. Z. 256 (1932) 134-144.

747. Koperina, A.W.: The resins of tobacco smoke; Vsesoy. Inst. Tabakhnoi Makharoch. Promysh. Publ. No. 109 (1934) 99-106.

748. Wenusch, A.: Über das Vorkommen von Harzen im Tabakrauch [The occurrence of resins in tobacco smoke]; Z. Untersuch. Lebensm. 69 (1935) 81-85.

749. Wenusch, A.: Über die nichtflüchtigen Bestandteile des Tabakrauchs [Composition of the non-volatile constituents of tobacco smoke]; Pharm. Zentralhalle 78 (1937) 238-241.

750. Mazzulli, R.B.: Contribution to the study of the resin content of smoke from native tobaccos; Rev. Facultad Sci. Quim. (Quim. y Farm.) 25 (1950) 103-110.

751. Von Degrazia, J.: Verfahren zur Bestimmung der Tabakharze and seine Anwendung an verschiedenen Tabaken [Method for the determination of tobacco resin and its application to various tobaccos]; Fachl.. Mitt. Österr. Tabakregie (1914) 1-4.

752. Gaertner, K.: Grading of tobacco on the basis of chemical composition. I; Magyar Chemiai Folyoirat 44 (1938) 47-59.

753. Pyriki, C.: Über harzartige Bestandteile des Orient Tabaks und dessen Rauchs [Resinous constituents of Oriental tobacco and its smoke]; Z. Untersuch. Lebensm. 80 (1940) 42-61.

754. Dittmar, H.: The effect of saccharides in tobacco on some components of cigarette smoke; Tabak (Berlin) 1 (1940) 118-125.

755. Martin, A.L. and S.F. Trelease: Absorption of selenium by tobacco and soybeans in sand cultures; 
Am. J. Botany 25 (1933) 380-385.

756. MacIntire, W.H., W.M. Shaw, and J.B. Young: The role of silica in counteracting magnesia-induced toxicity; Soil Sci. 19 (1925) 331-341.

757. Frankenburg, W.G.: Chemical changes in the harvested tobacco leaf; Adv. Enzymology 10 (1950) 325-441.

758. Trimble, H.: The occurrence of strontium in plants; Am. J. Pharm. 69 (1897) 296

759. Shedd, O.M.: The relation of sulphur to soil fertility; Kentucky Sta. Bull. 188 (1914) 595-630.

760. McMurtrey, J.E.: Effect of thallium on growth of tobacco plants; Science 76 (1932) 86.

761. Van Der Veen, R.: Frenching of tobacco as a toxic symptom; Meded. Besock. Proefsta. 61 (1938) 15-20.

762. Shear, G.M. and H.D. Ussery: Frenching of tobacco distinguished from thallium toxicity by spectrographic analysis; J. Agr. Res. 60 (1940) 129-149.

763. Toth, J.: Thiocyanogen compounds in tobacco smoke; Chem. Ztg. 33 (1909) 1301-1302.

764. Cavalli, A.: Über die Verbrennungsprodukte des Tabaks [On the combustion products of tobacco]; Selmi 8 (1898) 25.

765. Murray, B.J.A.: Nicotiana tabacum; Apparatus Medicam. (1793) 681-702.

766. Carpenter, F.B.: Types of tobacco and their analyses; North Carolina Experiment Station, Bull. 122 (1895) 331-366.

767. Kissling, R.: Handbuch der Tabakkunde, des Tabakbaues, und der Tabakfabrikation [Handbook of tobacco science, tobacco cultivation, and tobacco manufacture]; Paul Parey, Berlin, Germany (1905).

768. Lehmann, K.B.: Vorläufiger Bericht über Tabakuntersuchungen [Preliminary reports on tobacco studies]; Hyg. Rundschau 17 (1907) 1100-1101.

769. Kissling, R.: Fortschritte der Tabakchemie [Progress in tobacco chemistry]; Chem. Ztg. 32 (1908) 717-718.

770. Kissling, R.: Fortschritte auf dem Gebiete der Tabakchemie [Progress in the field of tobacco chemistry]; Chem. Ztg. 34 (1910) 486-488.

771. Ehrenstein, M.: Neuere Entwicklungen in Chemie und Biochemie des Tabaks [New developments in the chemistry and biochemistry of tobacco]; Arch. Pharm. 268 (1930) 430-433.

772. Gabelya, Y.O. and G.I. Kipriyanov: Reaction of tobacco smoke as an index of the quality of tobacco; Ukrainskii Khem. Zhur. 5 Tech. Pt. (1930) 167-184.

773. Shmuk, A.A. (editor): Research on the chemistry of tobacco; Gosudartsvennyi Inst. Tabakovedeniya Publ. No. 80 (1931).

774. Waser, E.: Über Tabak und Tabakprodukte [Tobacco and tobacco products]; Mitt. Gebiet Lebensm. Hyg. 26 (1935) 205-211.

775. Wenusch, A.: Beitrag zur Kenntnis der Tabaksorten [Contribution to knowledge on tobacco varieties]; $\mathrm{Z}$. Untersuch. Lebensm. 70 (1935) 506-510.

776. Schöller, R. and E. Molinari: Beitrag zur Qualitätsbestimmung von Tabaken durch chemischphysikalische Untersuchungen. Teil II [Contribution to the determination of tobacco quality through physicochemical research. Part II]; Fachl. Mitt. Österr. Tabakregie 1937(2) 1-15.

777. Brandt, W.: Tabak und Tabakrauch. Teil I [Tobacco and tobacco smoke. Part I]; Chem. Ztg. 62 (1938) 841-843.
778. Brandt, W.: Tabak und Tabakrauch. Teil II [Tobacco and tobacco smoke. Part II]; Chem. Ztg. 62 (1938) 851-852.

779. Bodnár, J. and Z. Votisky: Measurement of tobacco quality on the basis of chemical composition of tobacco and the determination of quality through tobacco smoke reaction or "Smoke Number"; Z. Untersuch. Lebensm. 80 (1940) 515-527.

780. Smirnov, A.I., M.P. Platnisky, A.P. Smirnov, and A.A. Sirotenko: Biochemie des tabacs [The biochemistry of tobacco]; Utigeverij, The Hague (1940) pp. 4-64.

781. Palfray, L., S. Sabetay, L. Sabourin, and H.F. Emmanuel: Le tabac et son parfum [Tobacco and its aroma]; Ann. Chim., Anal. Chim. Appl. 23 (1941) 311.

782. Pyriki, C.: Information über Tabak und Tabakchemie für Jedermann [Tobacco information and tobacco chemistry for everybody]; Deut. Tabakztg. 12 (1943) 1; Deut. Tabakztg. 13 (1943) 2.

783. Pyriki, C.: Untersuchungen von neue angebauten Deutschen Tabaken [Investigation of newly generated German tobacco]; Z. Untersuch. Lebensm. 90 (1950) 276.

784. Baumberger, J.P.: Amount of smoke produced from tobacco and its absorption in smoking as determined by electrical precipitation; J. Pharm. Exp. Therap. 21 (1923) 47-57.

785. Koperina, A.W.: An investigation of the nitrogenous substances of tobacco smoke; in: Research on the chemistry of tobacco, edited by A.A. Shmuk, Gosudartsvennyi Inst. Tabakovedeniya Publ. No. 80 (1931) 61-75.

786. Kurilo, M.E.: Estimation of the aromatic substances in tobacco smoke; Vsesoyuznyi Institut Tabachnoi Promyshlennosti, 104 (1933) 33-39.

787. Tschebull, E.: Die Analyse des Zigarettenrauches [Analysis of cigarette smoke]; Österr. Chem. Ztg. 37 (1934) 127-128.

788. Pyriki, C.: Beiträge zur Frage des Tabakrauchens [Contributions to the question of tobacco smoking]; Pharm. Zentralhalle 78 (1937) 533-534.

789. Rohrbach, E.: Beiträge zur Kenntnis des Tabakrauchens [Contributions to knowledge of tobacco smoking]; Pharm. Zentralhalle 78 (1937) 453-463.

790. Wenusch, A.: Über die Abrauchmethode von B. Pfyl [On the smoking method of B. Pfyl]; Pharm. Zentralhalle 78 (1937) 189-191.

791. Smirnov, A.P. and A.A. Sirotenko: Studies on aromatic substances in tobacco smoke; Vsesoy. NauchnoIssled. Inst. Tabach 140 (1939) 103-108, see Shmuk, A.A.: Collected works on the chemistry of tobacco and Makhorka, Vol. 8 (1939).

792. Wenusch, A.: Der Tabakrauch. Seine Entstehung, Beschaffenheit und Zusammensetzung [Tobacco smoke. Its formation, quality, and composition]; Arthur Geist, Bremen, Germany (1939).

793. Hillsman, O.L.: Factors influencing the composition of cigarette smoke; Virginia Sci. J. 2 (1941) 195.

794. Matthews, J.E.: An investigation of certain factors affecting the composition of cigarette smoke; Thesis, Pennsylvania State College (1941) pp. 1-77.

795. Fromm, F.: On tobacco smoke; Science 104 (1946) 376.

796. Matthews, J.E.: Smoke technique and analysis; $2^{\text {nd }}$ Tobacco Chemists' Research Conference, Program Booklet and Abstracts, Vol. 2, Paper No. 8, 1948, p. 1. 
797.Laskowski, K.: Components of tobacco smoke and their absorption in the respiratory system of the smoker; Rocz. Panstwowego Zadlaku Hig. 2 (1951) 139-160.

798. Warner, B.R. and M.E. Hobbs: An investigation of the technique and methods for the study and evaluation of the physical and chemical properties of some aerosols; Progress Rept. No. 1, Damon Runyon Memorial Fund, Research Project DRIR-186 (1953).

799. Drobkov, A.A.: The role of natural radioactive elements in the life of plants; Soviet. Agron. 7(9) (1949) 75-79, see Chem. Abstr. 44 (1949) 708.

800.Phillips, M. and A.M. Bacot: The content of uronic acids in several grades of flue-cured type 12 tobacco; J. Assoc. Off. Agr. Chem. 36 (1953) 123-128.

801. Barnhardt, W.W.: Moisture determination; $2^{\text {nd }}$ Tobacco Chemists' Research Conference, Program Booklet and Abstracts, Vol. 2, Paper No. 6, 1948.

802. Roffo, A.H.: Desarrollo de un carcinome en el conejo por el tabaco [Development of a carcinoma in a rabbit by tobacco]; Bol. Inst. Med. Exptl. Estud. Cáncer 7 (1930) 501-538.

803. Kennaway, E.L. and I. Hieger: Carcinogenic substances and their fluorescent spectra; Brit. Med. J. 1930(i) 1044-1046.

804. Cook, J.W., C.L. Hewitt, and I. Hieger: Isolation of a cancer-producing hydrocarbon from coal tar. II. Isolation of 1,2- and 4,5-benzpyrenes, perylene, and 1,2-benzanthracene; J. Chem. Soc. (1933) 395-398.

805. Eby, J.: Personal communication to Wynder, Graham, and Croninger (1953).

806. Fieser, L.F.: Chemical carcinogenesis; Arthur Stoll Festschriffte (1957) 489-498.

807. Wynder, E.L. and D. Hoffmann: Experimental tobacco carcinogenesis; Adv. Cancer Res. 8: (1964) 249-453, see 259

808. Wynder, E.L. and D. Hoffmann: Tobacco and tobacco smoke: Studies in experimental carcinogenesis; Academic Press, New York, NY (1967).

809. Newsome, J.R. and C.H. Keith: Variation of the gas phase composition within a burning cigarette; Tob. Sci. 9 (1965) 65-69.

810.Larson, P.S., H.B. Haag, and H. Silvette: Tobacco: Experimental and clinical studies, Williams and Wilkins Company, Baltimore, MD (1961) pp. 422-424.

811. Roffo, A.E.: El CO en los enfermos con carcinomas [CO in those ill with carcinoma]; Bol. Inst. Med. Exptl. Estud. Cáncer 18 (1941) 559-565.

812. Roffo, A.E.: Espectrograffe de los derivados obtenidos por destilacion directa de los tabacos y su relacion como agentes carcinogenos [Spectrograph of derivatives obtained by direct distillation of tobaccos and the relation to carcinogenic agents]; Bol. Inst. Med. Exptl. Estud. Cáncer 17 (1940) 279.

813. Roffo, A.H.: Cancerización gástrica por ingestion de alquitrán tabáquico [Gastric cancer by ingestion of tobacco tar]; Bol. Inst. Med. Exptl. Estud. Cáncer 18 (1942) 39-68, see Chem. Abstr. 36 (1942) 1433.

814. Cooper, R.L. and A.J. Lindsey: 3,4-Benzpyrene and other polycyclic hydrocarbons in cigarette smoke; Brit. J. Cancer 9 (1955) 304-309.

815. Lefemine, D.V., E.T. Alvord, and S.Z. Cardon: Identification of 3,4-benzpyrene in cigarette paper smoke and tars; Southeastern Reg. Mtg., Am. Chem. Soc.,
Birmingham, AL (1954).

816. Cardon, S.Z. and E.T. Alvord: The presence of 3,4benzpyrene in cigarette smoke; Ann. Mtg., Am. Assoc. Adv. Sci., Atlanta, GA (1955) pp. 1-12.

817. Alvord, E.T. and S.Z. Cardon: Separation and identification of 3,4-benzpyrene in cigarette smoke; $9^{\text {th }}$ Tobacco Chemists' Research Conference, Program Booklet and Abstracts, Vol. 9, Paper No. 24, 1955, p. 13.

818. Wynder, E.L. and D. Hoffmann: The role of higher polycyclic hydrocarbons in tobacco carcinogenesis; Proc. Am. Assoc. Cancer Res. 3 (1) (1959) 74; A study of tobacco carcinogenesis. VII. The role of higher polycyclic hydrocarbons; Cancer 12 (1959) 1079-1086.

819. Rodgman, A. and L.C. Cook: The analysis of cigarette smoke condensate. XIV. Polycyclic aromatic hydrocarbons; RDR, 1960, No. 20, May 26, see www.rjrtdocs.com $501008592-8660$.

820. Rodgman, A.: Studies of polycyclic aromatic hydrocarbons in cigarette mainstream smoke: Identification, tobacco precursors, control of levels: A review; Beitr. Tabakforsch. Int. 19 (2001) 361-379.

821.Roffo, A.H.: Durch Tabak beim Kaninchen entwickeltes Carcinom [Initiation of carcinomas in rabbits with tobacco]; Z. Krebsforsch. 33 (1931) 321-332.

822. Roffo, A.H.: Carcinome dévelopé chez un lapin après trois ans, par l'effet du tabac [Carcinoma developed in a rabbit after three years due to the effect of tobacco]; Néoplasmes 2 (1932) 77-89.

823. Roffo, A.H.: Carcinome tabáquico de gran malignidad desarrollado en un conejo [Tobacco carcinoma of great malignancy developed in a rabbit]; Bol. Inst. Med. Exptl. Estud. Cáncer 20 (1943) 1-48.

834.Roffo, A.H.: Cáncer experimental desarrollado por alquitrán de tabaco. Sarcomo fusocelular [Experimental cancer produced by tobacco tar. Fusocellular sarcoma]; Bol. Inst. Med. Exptl. Estud. Cáncer 19 (1942) 419-430.

825. Roffo, A.H.: Unidad cancerígena de los alquitranes de diversos tipos de tabacos [Uniform carcinogenicity of the tars from different types of tobacco]; Rev. Liga Puertorriq. Contra el Cáncer 3 (1943) 122-124.

826. Wynder, E.L. and D. Hoffmann: Ein experimenteller Beitrag zur Tabakrauchkanzerogenese [An experimental contribution to tobacco smoke carcinogenesis]; Deut. Med. Wchnschr. 88 (1963) 623-628.

827. Roffo, A.H.: Correlación del valor cancerígeno del alquitrán de tabaco con el de hulla [Correlation of the carcinogenicity of tobacco tar with that from soft coal]; Bol. Inst. Med. Exptl. Estud. Cáncer 17 (1940) 699-713, see Chem. Abstr. 35 (1941) 37093.

828. Jaffe, R., W. Jaffe, and L. Potenza: Acción cancerígena de alquitran de cigarillo y de chimo [Carcinogenic action of cigarillo tar and its chemistry]; Rev. San. (Caracas) 11 (1946) 387-390.

829. Hueper, W.C.: Role of occupational and environmental air pollutants in production of respiratory cancers; Arch. Path. 63 (1957) 427-450.

830. Rodgman, A.: The analysis of cigarette smoke condensate. II. The pretreatment of Camel blend tobacco; RDR, 1956, No. 12, November 1, see www.rjrtdocs.com 501008294 -8336, 504912107 2148, $515839741-9783$. 
831. Rodgman, A.: The analysis of cigarette smoke condensate. VI. The influence of solvent pretreatment of tobacco and other factors on the polycyclic hydrocarbon content of smoke condensate; RDR, 1959, No. 1, January 29, see www.rjrtdocs.com $501008529-8591$.

832. Ashburn, J.G.: Study of tobacco pretreatments; RDR, 1958, No. 20, December 10, see www.rjrtdocs.com $504912251-2337$.

833. Wynder, E.L. and G.F Wright: Studies on the identification of carcinogens in cigarette tar; Proc. Am. Assoc. Cancer Res. 2(2) (1956) 159; A study of tobacco carcinogenesis. I. The primary fractions; Cancer 10 (1957) 255-271.

834. Grossman, J.D. and R.L. Stedman: Composition studies on tobacco. II. Isolation and identification of stigmasterol from flue-cured leaves; Tob. Sci. 2 (1958) 115-116.

835. Dymicky, M. and R.L. Stedman: Composition studies on tobacco. IV. Ergosterol, $\gamma$-sitosterol and a partially characterized steroidal glycoside from flue-cured leaves; Tob. Sci. 3 (1959) 4-8.

836. Dymicky, M. and R.L. Stedman: Composition studies on tobacco. IX. Campesterol from flue-cured leaves; Tob. Sci. 3 (1959) 179-181.

837.Cook, C.E., M.E. Twine, C.R. Tallent, I. Harper, G. Heunisch, J.B. Lewis, and M.E. Wall: An examination of the hexane extract of flue-cured tobacco involving gel permeation chromatography; Phytochemistry 8 (1969) 1025.

838. Grunwald, C., D.L. Davis, and L.P. Bush: Cholesterol in cigarette smoke condensate; J. Agr. Food Chem. 19 (1971) 138-139.

839. Rodgman, A., L.C. Cook, and P.H. Latimer: The analysis of cigarette smoke condensate. VIII. Solanesyl esters and phytosteryl esters; RDR, 1959, No. 2, February 10, see www.rjrtdocs.com 500933072 -3093; The composition of cigarette smoke. II. Solanesyl and phytosteryl esters; Tob. Sci. 3 (1959) 125-128.

840. Kosak, A.I., J.S. Swinehart, D. Taber, and B.L. Van Duuren: Stigmasterol in cigarette smoke; Science 125 (1957) 991-992.

841. Carruthers, W. and R.A.W. Johnstone: Phytosterols in cigarette smoke; Chem. and Ind. (London) (1958) 1663-1664.

842. Severson, R.F., W.S. Schlotzhauer, O.T. Chortyk, R.F. Arrendale, and M.E. Snook, Precursors of polynuclear aromatic hydrocarbons in tobacco smoke; in: $3^{\text {rd }}$ International Symposium on Carcinogenesis and Mutagenesis, edited by P.W. Jones and P. Leber, Ann Arbor Science, Ann Arbor, MI (1979) 277-298.

843. Rodgman, A. and L.C. Cook: The analysis of cigarette smoke condensate. $\mathrm{V}$. The polycyclic hydrocarbon precursors in tobacco; RDR, 1958, No. 18, December 1 , see www.rjrtdocs.com $501008387 \quad-8441$, $504912197-2250$.

844. Rowland, R.L., A. Rodgman, J.N. Schumacher, D.L. Roberts, L.C. Cook, and W.E. Walker Jr: Macrocyclic diterpenes. Hydroxyethers from tobacco and tobacco smoke; $17^{\text {th }}$ Tobacco Chemists' Research Conference, Program Booklet and Abstracts, Vol. 17, Paper No. 16, 1963, p. 13; Macrocyclic diterpene hydroxyethers from tobacco and cigarette smoke; J. Org. Chem. 29 (1964) 16-21.

845.Falk, H.L., S. Goldfein, and P. Steiner: The products of cholesterol at $360{ }^{\circ} \mathrm{C}$ and their relation to carcinogens; Cancer Res. 9 (1949) 438-447.

846.Beck, S., A.H.M. Kirby, and P.R. Peacock: Tumors induced with heated cholesterol; Cancer Res. 5 (1945) 135-139.

847. Veldstra, H.: 3,5-Cholestadiene from cholesteryl oleate and its possible bearing upon the formation of carcinogenic substances in heated fats; Nature 144 (1939) 246-247.

848. Cohen, A., J.W. Cook, and C.L. Hewett: The synthesis of compounds related to the sterols, bile acids, and oestrus-producing hormones. Part VI. Experimental evidence of the complete structure of oestrin, equilin, and equilenin; J. Chem. Soc. (1935) 445-455.

849. International Agency for Research on Cancer (IARC): Evaluation of the carcinogenic risk of chemicals to humans: Polynuclear aromatic hydrocarbons. Part 1. Chemical, environmental and experimental data; IARC, Lyon, France, IARC Monograph 32 (1983).

850. Hartwell, J.L.: Survey of compounds which have been tested for carcinogenic activity; USPHS Publ. No. 149, $2^{\text {nd }}$ Edition, Washington, DC (1951).

851.Roffo, A.H.: Malignant tumors of the gastrointestinal tract from feeding with heat-oxidized fats; Bull. Assoc. Franc. Cáncer 28 (1939) 556-558.

852. Roffo, A.H.: Malignant tumors of the gastrointestinal tract produced by ingestion of fats oxidized by heat; Bol. Inst. Med. Exptl. Estud. Cáncer 15 (1939) 407-521, see Chem. Abstr. 33 (1939) 54787

853. Peacock, P.R. and S. Beck: Multiple mesenteric sarcomata in rats following ingestion of heated lard; Brit. J. Exp. Path. 24 (1943) 143, see Chem. Abstr. 38 (1944) $1275^{3}$

854.Lane, A., D. Blickenstaff, and A.C. Ivy : The carcinogenicity of fat "browned" by heating; Cancer 3 (1950) 1044-1051.

855. Morris, H.B., C.D. Larsen, and S.J. Lippincott: Effect of feeding heated lard to rats. Histological description of lesions produced; J. Natl. Cancer Inst. 4 (1943) 285.

856.Lam, J.: Determination of 3,4-benzpyrene and other aromatic compounds formed by pyrolysis of aliphatic tobacco hydrocarbons; Acta Pathol. Microbiol. Scand. 39 (1956) 207-210.

857. Badger, G.M., J.K. Donnelly, and T.M. Spotswood: The formation of aromatic hydrocarbons at high temperatures. XXIV. The pyrolysis of some tobacco constituents; Australian J. Chem. 18 (1965) 1249-1266.

858. Halaby, G.A. and I.S. Fagerson: Heat-treated food constituents; Proc. $3^{\text {rd }}$ Internat. Cong. Food Sci. Technol., Washington, DC (1970) 820.

859. Kitamura, K.: Pyrolysis of triglycerides; Bull. Chem. Soc. Japan 44 (1971) 1606-1609.

860. Higman, E.B., I. Schmeltz, H.C. Higman, and O.T. Chortyk: Studies on the thermal degradation of naturally occurring materials. II. Products from the pyrolysis of triglycerides at $400{ }^{\circ} \mathrm{C}$; J. Agr. Food Chem. 21 (1973) 202-204.

861 Roffo, A.H.: Alquitrán de te cancerígeno [Carcinogenic tea tar]; Bol. Inst. Med. Exptl. Estud. Cáncer 17 (1940) 661-699, see Chem. Abstr. 35 (1941) 37092.

862.Roffo, A.H.: Alquitrán cancerígeno de Yerba mate [Carcinogenic tar from yerba mate]; Bol. Inst. Med. Exptl. Estud. Cáncer 18 (1941) 5-37.

863. Kuratsune, M.: Benzo[a]pyrene content of certain 
pyrogenic materials; J. Natl. Cancer Inst. 16 (1956) 1485-1496.

864. Kuratsune, M. and W.C. Hueper: Polycyclic hydrocarbons in coffee soot; J. Natl. Cancer Inst. 20 (1958) 37-51.

865. Maga, J.A.: Potential health hazards associated with smoke; Chapter $10 \mathrm{in}$ : J.A. Maga: Smoke in food processing, CRC Press, Boca Raton, FL (1988) 113-144.

866. Roffo, A.H.: Carcinogenic tar from coffee; Bull. Assoc. Franc. Cancer 28 (1939) 910-924.

867. Widmark, E.M.P.: Presence of cancer producing substances in roasted food; Nature 143 (1939) 984.

868. Proctor, R.N.: Angel H. Roffo: The forgotten father of experimental tobacco carcinogenesis; Bull. World Hlth. Org. 84 (2006) 494-496.

869. Clayton, E.E.: Resistance of tobacco to blue mold; J. Agr. Res. 70 (1945) 79-85.

870. Garner, W.W.: The production of tobacco; Revised $1^{\text {st }}$ Ed., The Blakiston Co., New York, NY (1951) pp. 234-300.

871. Chamberlin, F.S. and A.H. Madden: Progress report on dusts containing rotenone for the control of flea beetles attacking shade-grown cigar-wrapper tobaccos; The Florida Entomologist 20 (1937) 25-29.

872.LaForge, F.B. and H.L. Haller: Constituents of pyrethrum flowers. V. Concerning the structure of pyrethrolone; J. Am. Chem. Soc. 58 (1936) 1061.
873. LaForge F.B. and W.F. Barthel: Constituents of pyrethrum flowers. XVI. Heterogeneous nature of pyrethrolone; J. Org. Chem. 9 (1944) 242-249.

874. Moore, W. and S.A. Graham: A neglected factor in the use of nicotine sulphate as a spray; J. Agr. Res. 10 (1917) 47-50.

875. Staudinger, H. and L. Ruzicka: Insektentotende Stoffe. I-VI and VIII-X [Insecticidal material. I-VI and VIIIX]; Helv. Chim. Acta 7 (1924) 177-259, 377-390, 406-458.

876. Diaz del Castillo, B.: Historia verdadera de la conquista de la neuva-Espaňa [True history of the conquest of the New Spain]; Madrid (1632).

877. Garner, W.W.: The production of tobacco; Revised $1^{\text {st }}$ Ed., The Blakiston Co., New York, NY (1951) pp. 464-465.

Corresponding author:

Alan Rodgman

2828 Birchwood Drive,

Winston-Salem,

North Carolina, 27103-3410,

USA

E-mail:arodgman@triad.rr.com 